\title{
MINIMUM-WEIGHT DESIGN OF COMPRESSIVELY LOADED STIFFENED PANELS FOR POSTBUCKLING RESPONSE
}

\author{
by
}

\section{Christine Ann Perry}

Thesis submitted to the Faculty of the

Virginia Polytechnic Institute and State University

in partial fulfillment of the requirements for the degree of

\section{MASTER OF SCIENCE}

in

Engineering Mechanics

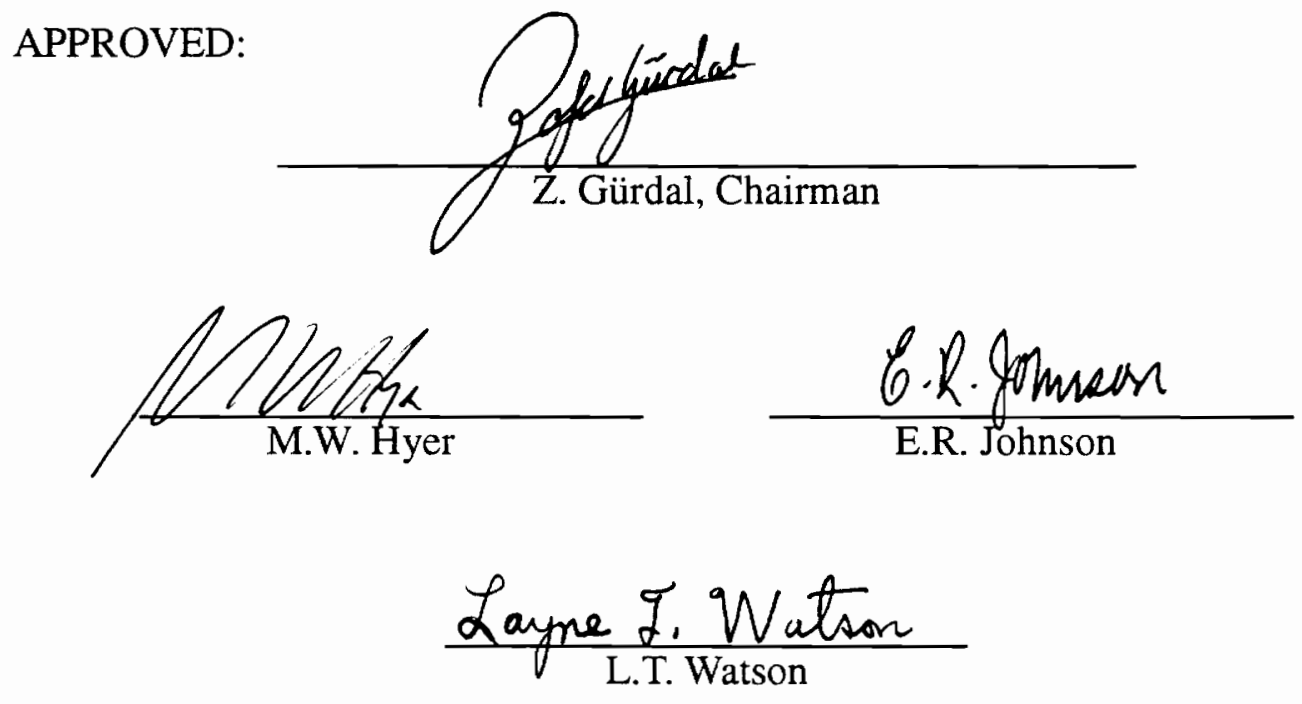

March, 1995

Blacksburg, Virginia

Key Words: Buckling, Composite Design, Imperfection Sensitivity, Postbuckling, Stiffened Panels 
c.

$\triangle D$

405

$\sqrt{855}$

1995

P477

Ci 


\title{
MINIMUM-WEIGHT DESIGN OF COMPRESSIVELY LOADED STIFFENED PANELS FOR POSTBUCKLING RESPONSE
}

\author{
by \\ Christine Ann Perry \\ Committee Chair: Dr. Zafer Gürdal \\ Engineering Mechanics
}

(ABSTRACT)

A computationally efficient procedure, NLPANOPT, is developed for the preliminary design of minimum-weight thin-walled stiffened composite panels loaded in uniaxial compression based on a geometrically nonlinear analysis. An approximate, semi-analytical nonlinear analysis code, NLPAN, which requires buckling eigenfunction information from the buckling analysis code, VIPASA, is linked with the optimization code ADS. A blade-stiffened and T-stiffened panel are designed for specified loads using NLPANOPT for postbuckling response and PASCO for buckling-critical response. Comparisons of panel weight and imperfection sensitivity between the NLPANOPT designs and PASCO designs are presented. In general, the designs obtained with NLPANOPT are lighter and less imperfection sensitive than the designs obtained with PASCO. The nonlinear analysis allows for a more accurate prediction of the true strength of the stiffened structure, by accounting for postbuckling strength and modal interaction. The effect of laminate stacking sequence is also investigated. The current design procedure requires the stacking sequence to be prescribed, proving to be a limitation in the design procedure. 


\section{Acknowledgements}

This material is based upon work supported under a National Science Foundation Graduate Fellowship. Any opinions, findings, conclusions or recommendations expressed in this publication are those of the author and do not necessarily reflect the views of the National Science Foundation. This study was also supported by the NASA-Virginia Tech Composites Program under NASA Grant NAG-1-643.

The author would like to thank Dr. Gürdal and Dr. James H. Starnes, Jr. for their advice and direction throughout this work, along with Dr. Hyer, Dr. Johnson, and Dr. Watson for their time and interest. The work of Dr. Frederick Stoll on the nonlinear analysis method used in this design procedure and his continuous support is greatly appreciated. Thanks are also due to Nagendra Somanath, Scott Ragon, and Brian Tatting for their input and assistance. Finally, the author would like to thank her family for their support from the very beginning. 


\section{Table of Contents}

1.0 Introduction and Literature Review ......................................................1

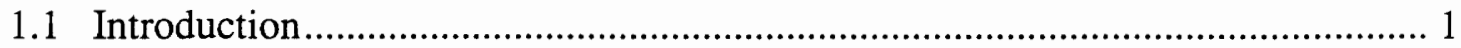

1.2 Literature Review.............................................................................. 5

2.0 Analysis ................................................................................................. 11

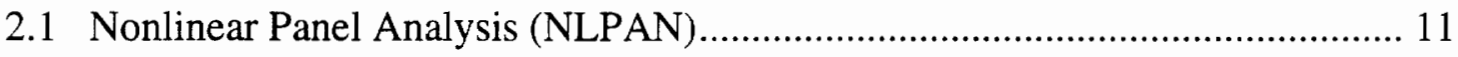

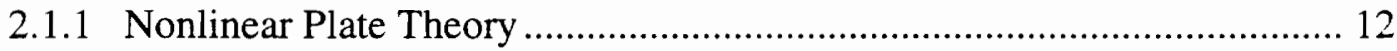

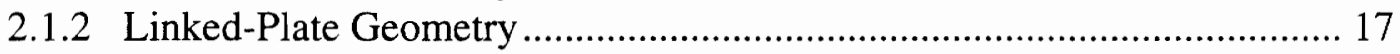

2.1.3 Expansions of Displacement Functions ........................................ 21

2.1.4 Solution of Equations................................................................. 27

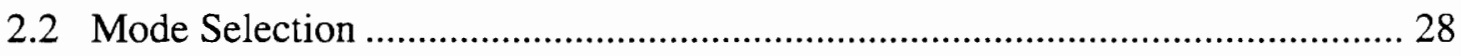

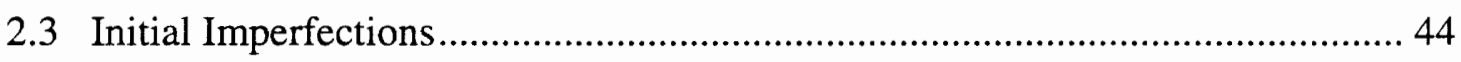

3.0 Design Formulation .................................................................47

3.1 Program Organization ...................................................................... 47

3.2 Optimization Program - ADS ............................................................... 49

3.3 Statement of Minimization Problem ......................................................... 49

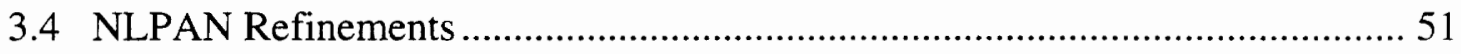

3.4.1 Material Failure Constraint ............................................................. 51

3.4.2 Gradient Calculations..................................................................... 52

4.0 Design Study ...........................................................................55

4.1 Design of Blade-Stiffened Panel for Four Levels of Axial Compressive Loads 55

4.1.1 Case 1: 100 lb/in Design Load............................................................. 57

4.1.2 Case 2: $1000 \mathrm{lb} / \mathrm{in}$ Design Load ................................................... 58

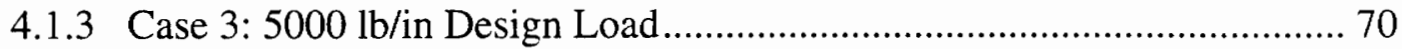

4.1.4 Case 4: $10000 \mathrm{lb} / \mathrm{in}$ Design Load ..................................................... 86

4.2 Effect of Skin Laminate Stacking Sequence .......................................... 90

4.3 Design of Blade-Stiffened Panel with Flanges ......................................... 98

4.3.1 $1000 \mathrm{lb} / \mathrm{in}$ Design Load............................................................... 100 
5.0 Concluding Remarks ..........................................................................114

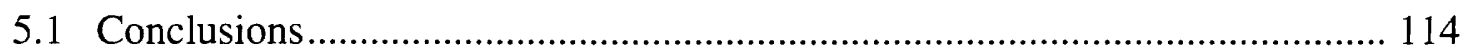

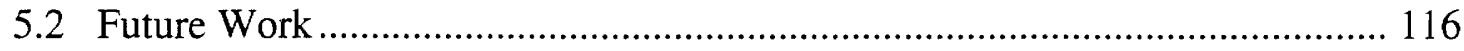

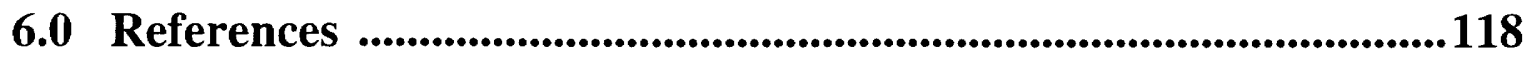

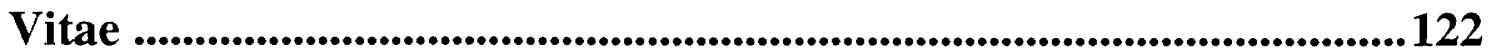

Table of Contents $\quad \mathrm{v}$ 


\section{List of Figures}

Figure 1. Nonlinear elastic behavior of stiffened panels. ...................................... 2

Figure 2. Typical compressively loaded prismatic linked-plate structures.................... 4

Figure 3. Convention of coordinate system. ........................................................... 13

Figure 4. Stiffened panel illustrating plate-strip numbers, node line numbers, and labeling conventions: Note that the local plate-strip axes shown do not reflect actual locations of origin. [4]

Figure 5. Example normalized eigenvalues scanned for selection in NLPANOPT mode sets.

Figure 6. Cross-sectional buckling mode shapes at panel midlength for half-wavelength number 8 for example eigenvalues plotted in Figure 5 .

Figure 7. Blade-stiffened panel geometry and possible parameters. (a) Eight blade-stiffened composite panel. (b) Stiffener-unit representation with sample parameters. All dimensions are in inches. 34

Figure 8. Parametric study of elastic limit load with and without symmetric global mode in mode set. 36

Figure 9. Cross-sectional buckling mode shapes at panel midlength for a blade height of 2.6 in. in Figure 8. 37

Figure 10. Comparison of modal amplitudes for Mode Set I (open symbols) and Mode Set II (filled symbols) at a blade height of $2.6 \mathrm{in.}$ 38

Figure 11. (a) Comparison of predicted elastic limit load using Mode Set II, Mode Set III, Mode Set IV, and Mode Set V for a range of $0^{\circ}$ blade ply thicknesses. (b) Close-up of region with largest discrepancies between mode sets (critical local half-wavelength numbers are shown by bold numbers next to each point). 40

Figure 12. Comparison of predicted maximum axial strain in panel using Mode Set II, Mode Set III, Mode Set IV, and Mode Set V for a range of $0^{\circ}$ blade ply thicknesses. . 42

Figure 13. Comparison of predicted elastic limit load using Mode Set II, Mode Set III, and Mode Set $\mathrm{V}$ for a range of $0^{\circ}$ blade ply thicknesses. 
Figure 14. Parametric study of elastic limit load using Mode Set I with a positive and negative global imperfection shape. 46

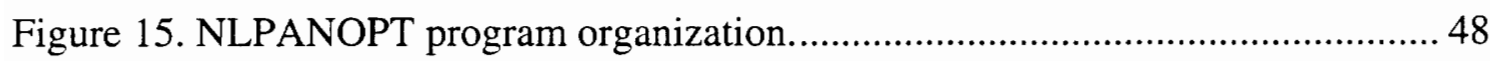

Figure 16. Possible panel responses for constraint value determination. ...................... 53

Figure 17. Imperfection sensitivity of the rounded-off PASCO design with no initial imperfections $($ Design load $=1000 \mathrm{lb} / \mathrm{in})$.

Figure 18. Imperfection sensitivity of the rounded-off NLPANOPT design with no initial imperfections (Design load $=1000 \mathrm{lb} / \mathrm{in}$ ).

Figure 19. Imperfection sensitivity of the continuous PASCO design with no initial imperfections to global imperfection shapes (Design load $=1000 \mathrm{lb} / \mathrm{in}$ ). 64

Figure 20. Comparison of imperfection sensitivity between the continuous and rounded-off NLPANOPT design with no imperfections using NLPAN (Design load $=1000 \mathrm{lb} / \mathrm{in}$ ). 65

Figure 21. Imperfection sensitivity of the rounded-off PASCO design with initial bowing imperfection (Design load $=1000 \mathrm{lb} / \mathrm{in}$ ). 66

Figure 22. Imperfection sensitivity of the rounded-off NLPANOPT design with initial global imperfection shape (Design load $=1000 \mathrm{lb} / \mathrm{in}$ ). 68

Figure 23. Comparison of imperfection sensitivity between the continuous and rounded-off NLPANOPT design with initial global imperfection shape using NLPAN $($ Design load $=$ $1000 \mathrm{lb} / \mathrm{in})$. 69

Figure 24. Imperfection sensitivity of PASCO design with no initial imperfections (Design load $=5000 \mathrm{lb} / \mathrm{in})$. 72

Figure 25. Imperfection sensitivity of NLPANOPT design with no initial imperfections (Design load $=5000 \mathrm{lb} / \mathrm{in}$ ). 73

Figure 26. Weight and blade height trends of multiple optima for NLPANOPT designs with no initial imperfections (Design load $=5000 \mathrm{lb} / \mathrm{in}$ ). 74

Figure 27. Panel response of multiple optima for NLPANOPT designs with no initial imperfections (Design load $=5000 \mathrm{lb} / \mathrm{in}$ ). 75

Figure 28. Imperfection sensitivity of Design 2 obtained from NLPANOPT design with no initial imperfections (Design load $=5000 \mathrm{lb} / \mathrm{in}$ ) 77

Figure 29. Imperfection sensitivity of Design 3 obtained from NLPANOPT design with no initial imperfections (Design load $=5000 \mathrm{lb} / \mathrm{in}$ ). 78

Figure 30. Imperfection sensitivity of PASCO design with initial bowing imperfection (Design load $=5000 \mathrm{lb} / \mathrm{in}$ ) 81

Figure 31. Imperfection sensitivity of NLPANOPT design with initial global imperfection shape $($ Design load $=5000 \mathrm{lb} / \mathrm{in})$. 82

Figure 32. Panel response for multiple optima of NLPANOPT designs with initial global 
imperfection shape (Design load $=5000 \mathrm{lb} / \mathrm{in})$.

Figure 33. Imperfection sensitivity of Design 2 for NLPANOPT design with an initial global imperfection shape $($ Design load $=5000 \mathrm{lb} / \mathrm{in}$ ). 84

Figure 34. Load vs. end shortening for PASCO and NLPANOPT designs with no initial imperfections (Design load $=10000 \mathrm{lb} / \mathrm{in}$ ).

Figure 35. Imperfection sensitivity of PASCO design with no initial imperfections (Design load $=10000 \mathrm{lb} / \mathrm{in})$. 88

Figure 36. Imperfection sensitivity of NLPANOPT design with no initial imperfections (Design load $=10000 \mathrm{lb} / \mathrm{in}$ ).

Figure 37. Imperfection sensitivity of PASCO design with initial bowing imperfection (Design load $=10000 \mathrm{lb} / \mathrm{in}$ ). 91

Figure 38. Imperfection sensitivity of NLPANOPT design with initial global imperfection shape $($ Design load $=10000 \mathrm{lb} / \mathrm{in}$ ).

Figure 39. Comparison of imperfection sensitivity between the continuous and rounded-off NLPANOPT designs with initial global imperfection shape using NLPAN (Design load = $10000 \mathrm{lb} / \mathrm{in})$.

Figure 40. Load versus end shortening of rounded-off designs for blade-stiffened panel with different skin laminate stacking sequences (Design load $=1000 \mathrm{lb} / \mathrm{in}$ ).............. 96

Figure 41. T-stiffened panel geometry and possible design variables. 99

Figure 42. Imperfection sensitivity of the rounded-off PASCO design with no initial imperfections for panel with added flange (Design load $=1000 \mathrm{lb} / \mathrm{in}$ ). 102

Figure 43. Imperfection sensitivity of the continuous PASCO flange design with no initial imperfections to global imperfection shapes (Design load $=1000 \mathrm{lb} / \mathrm{in}$ ). 104

Figure 44. Imperfection sensitivity of the rounded-off NLPANOPT flange design with no initial imperfections (Design load $=1000 \mathrm{lb} / \mathrm{in}$ ). 105

Figure 45. Imperfection sensitivity of the continuous NLPANOPT flange design with no initial imperfections to global imperfection shapes (Design load $=1000 \mathrm{lb} / \mathrm{in}$ ). 106

Figure 46. Imperfection sensitivity of the rounded-off PASCO flange design with bowing imperfection (Design load $=1000 \mathrm{lb} / \mathrm{in}$ ). 107

Figure 47. Imperfection sensitivity of the NLPANOPT flange design with initial global imperfection (Design load $=1000 \mathrm{lb} / \mathrm{in}$ ). 108

Figure 48. Imperfection sensitivity of the continuous NLPANOPT flange design with initial imperfection to global imperfection shapes (Design load $=1000 \mathrm{lb} / \mathrm{in}$ ).

Figure 49. Load vs. End Shortening for NLPANOPT flange designs with initial global imperfection $\left(\mathrm{N}_{\mathrm{x}}=1000 \mathrm{lb} / \mathrm{in}\right)$. 111 


\section{List of Tables}

Table 1. Options for Controlling the Inplane Loading. [4] …........................................ 20

Table 2. Summary of Mode Sets (X indicates mode included, * indicates number of first secondary mode having the same symmetry as the primary mode) ………………..... 32

Table 3. Typical Graphite-Epoxy Lamina Material Values ........................................ 33

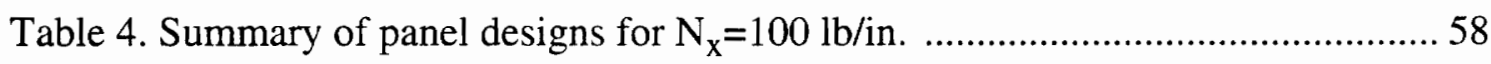

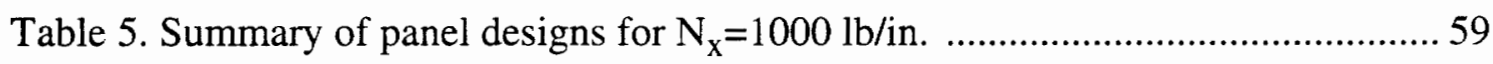

Table 6. Summary of panel designs for $\mathrm{N}_{\mathrm{x}}=5000 \mathrm{lb} / \mathrm{in}$. ........................................... 70

Table 7. Summary of NLPANOPT designs with no initial imperfections for $\mathrm{N}_{\mathrm{x}}=5000 \mathrm{lb} /$

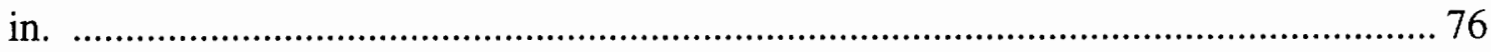

Table 8. Summary of NLPANOPT designs with initial global imperfection shape for $\mathrm{N}_{\mathrm{x}}=5000 \mathrm{lb} / \mathrm{in}$.

Table 9. Summary of panel designs for $\mathrm{N}_{\mathrm{x}}=10000 \mathrm{lb} / \mathrm{in}$. ........................................ 86

Table 10. Effect of skin stacking sequence on panel response of $[ \pm 45 / 90 / 0]$ skin laminate design. 95

Table 11. Summary of NLPANOPT designs for different skin laminate stacking sequences (Design Load $=1000 \mathrm{lb} / \mathrm{in}$ ). 97

Table 11. (Con't) Summary of NLPANOPT designs for different skin laminate stacking sequences $($ Design Load $=1000 \mathrm{lb} / \mathrm{in}$ ). 98

Table 12. Effect of skin stacking sequence on panel response of [0/ $\pm 45 / 90]$ skin laminate design. 100

Table 13. Summary of panel designs with flanges for $\mathrm{N}_{\mathrm{x}}=1000 \mathrm{lb} / \mathrm{in}$. 101

Table 14. Summary of NLPANOPT flange designs with an initial global imperfection for $\mathrm{N}_{\mathrm{x}}=1000 \mathrm{lb} / \mathrm{in}$. 


\subsection{Introduction and Literature Review}

\subsection{Introduction}

Upon buckling, the behavior of stiffened panels can be classified into three general types: local postbuckling, global (Euler) postbuckling, and an interaction between local and global modes termed modal interaction. The response of a perfect panel for each type of behavior is shown by the solid lines in Figure 1. For local postbuckling, where the panel generally buckles into half-wavelengths about equal to the width between stiffeners, the panel possesses postbuckling strength, carrying loads greater than its buckling load. For global postbuckling, where the panel buckles into one half-wavelength along its length, the panel's load-carrying capabilities remain essentially neutral after buckling. For modal interaction, where the local and global modes have critical loads of almost equal value, the panel is not able to carry loads greater than its buckling load. When imperfections are present, the load versus end shortening response of the panel is shown by the dashed lines in Figure 1. The local and global postbuckling behavior are not changed dramatically, but in the case of modal interaction, the panel reaches an elastic limit load below its buckling load, due to the imperfection sensitivity of the panel [1].

Aircraft structural panels are often designed for buckling response, $P=P_{c r}$. Such buckling-critical designs may be under-designed or over-designed. An under-designed panel is 


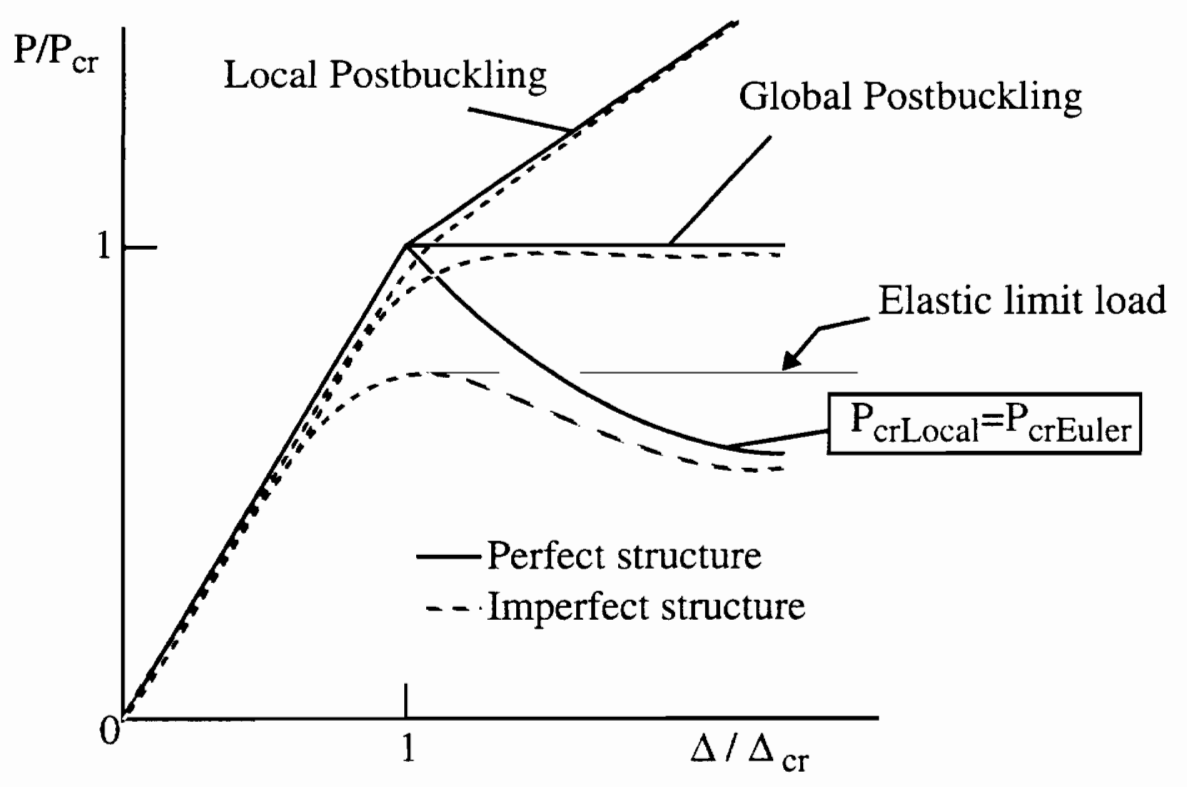

FIGURE 1. Nonlinear elastic behavior of stiffened panels. 
imperfection sensitive due to possible modal interaction behavior which reduces the loadcarrying capability of the panel dramatically. This behavior in buckling-critical designs is due to the eigenvalues of different eigenmodes having a common value, in a sense equal resistance is given to the possible failure modes. An over-designed panel is heavier than needed since postbuckling strength is not taken into account. Both shortcomings are avoided if stiffened panels are designed for performance in the postbuckling range. Large computer programs, for example STAGSC-1 [2,3], exist that have the capability of performing the necessary nonlinear structural analyses. The implementation of such a program into a design procedure, however, is computationally expensive, requiring costly nonlinear structural analyses to be performed repeatedly. The need for a computationally efficient nonlinear analysis has lead to the development of approximate methods for preliminary design.

An approximate, semi-analytical method was recently developed for the cost-effective, geometrically nonlinear analysis of thin-walled composite panels [4]. The analysis predicts nonlinear postbuckling stresses and deformations, elastic limit points, and imperfection sensitivity of panels subjected to a variety of loading conditions, including axial, biaxial, thermal, and pressure loadings. These panels are modelled as linked prismatic plate strips (see Figure 2) with compatibility satisfied at the plate strip junctions. The method was developed as an extension to the buckling analysis code VIPASA [5, 6] and uses a set of buckling eigenfunctions, calculated by VIPASA within the panel design code PASCO $[7,8]$, as the primary displacement shape functions for the nonlinear analysis. It has been incorporated into a FORTRAN code NLPAN (NonLinear Panel ANalysis) and is suitable for use in a design procedure because of its low computational cost. 

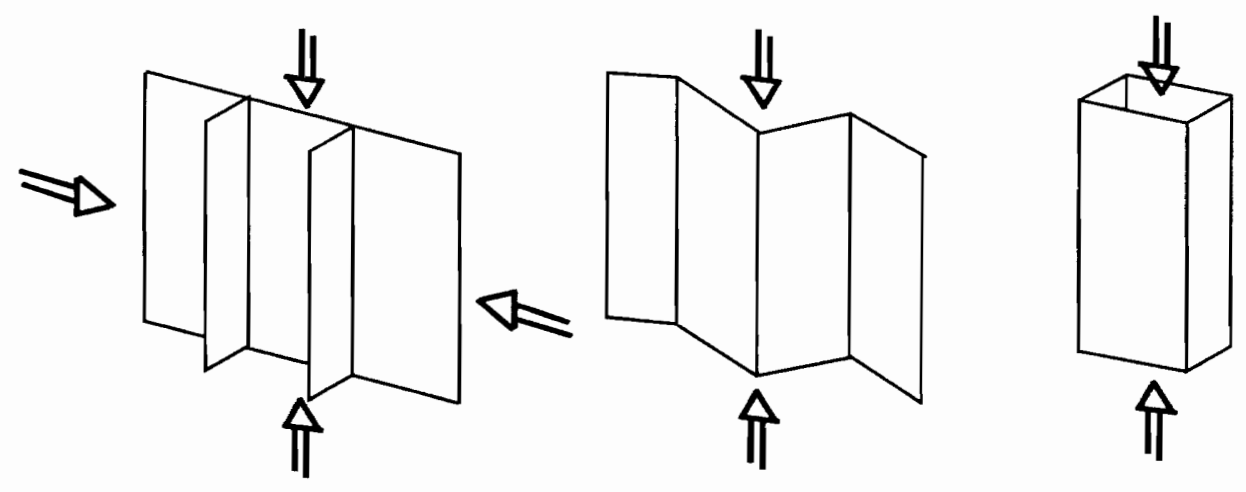

FIGURE 2. Typical compressively loaded prismatic linked-plate structures. 
The objective of this research is to study structural efficiencies and design trends of optimally designed, compressively loaded stiffened panels that have load-carrying capabilities in the local postbuckling region. The results are compared with stiffened panels designed for buckling response. In the present work, the NLPAN code is combined with the general-purpose optimization program ADS [9]. The complete integrated code is called NLPANOPT. Minimum-weight designs are sought for a specified load subject to constraints on global structural response and local material failure, with ply thickness and plate width variables. In comparison to buckling-constrained designs, these designs use a more accurate prediction of the true strength of a stiffened structure, by accounting for postbuckling strength and imperfection sensitivity.

A literature review of capabilities for buckling and postbuckling design completes Chapter 1 . A summary of the theory behind the nonlinear analysis used in this work is presented in Chapter 2. Included in this chapter is a discussion of two important aspects of the analysis, mode set selection and initial imperfections. Chapter 3 contains an explanation of the design procedure organization and formulation. Resuits of a simple blade-stiffened panel designed using NLPANOPT for four design loads are presented in Chapter 4. A comparison of weight and imperfection sensitivity is drawn between these designs and bucklingcritical designs obtained using PASCO. The effect of skin laminate stacking sequence and the addition of a flange to the stiffener on panel weight is presented for one design load. The final chapter, Chapter 5, includes conclusions drawn from the above design study and a discussion of future work.

\subsection{Literature Review}

With the incorporation of composite materials into structural design, the complexity of design options is greatly increased compared to designs using metallic materials. The vari- 
ability of ply thicknesses and orientations provide a disadvantage to establishing general design rules as was possible with metals. Thus, the need for an analysis which could account for all possible buckling modes arose. In 1976, the general-purpose, fully-automated buckling analysis code VIPASA [5, 6] was released. The analysis considers prismatic structures that are assembled by rigidly connecting thin plate-strips together along their longitudinal edges. Equilibrium conditions and displacement compatibilities are satisfied along the plate-strip junctions. The plate-strips are symmetric, balanced laminates and simply-supported along their ends. Possible loadings include biaxial inplane loading, shear loading, pressure, and temperature. The effect of an overall bowing imperfection is included. The analysis calculates the buckling load and mode for any given half-wavelength number exactly, in the context that the plate equations satisfy the Kirchoff-Love hypothesis and the panel ends are simply-supported. With the development of this analysis, approximate methods for predicting the buckling of stiffened panels, which considered possible buckling modes separate, were no longer required and a general design procedure was possible.

VIPASA was incorporated into the design code PASCO $[7,8]$, which uses the optimization code, CONMIN [10]. PASCO finds the minimum-weight design of stiffened composite panels subject to buckling and material constraints. Possible design variables include the plate widths, ply thicknesses, and ply orientations. Material failure is based on an assumed failure criterion. Options available for the failure criterion include maximum strain, maximum stress, or the Tsai-Wu criterion. Extensive studies comparing PASCO results with the EAL [11] and STAGSC-1 [2,3] finite element codes were performed by Stroud et al. [12]. An improvement to the VIPASA analysis' approach to buckling load calculations in the presence of inplane shear loadings was developed and implemented in the analysis code VICON (VIpasa with CONstraints) [13], which uses the method of 
Lagrange multipliers. The sizing code VICONOPT [14], which incorporated the buckling analysis code VICON, soon followed.

PASCO and VICONOPT aid considerably the general buckling design of stiffened composite panels. A structural-efficiency study of several graphite-epoxy rib components for a typical transport airplane wing was presented by Swanson, et al. [15]. PASCO was used to design corrugated panels, hat- and blade-stiffened, and unstiffened panels. Minimumweight designs satisfying buckling and strength constraints were obtained for various combinations of axial compression, inplane shear, and out-of-plane normal pressure loading.

Although a general buckling design code like PASCO has many advantages, there are also drawbacks to this design approach. The PASCO design process usually leads to configurations which have more than one buckling mode critical, result common to buckling critical designs. The optimizer forces the panel to have equal resistance to failure modes, in this case the global buckling mode and the local buckling mode. In the presence of imperfections, this design would likely fail below the buckling load due to the imperfection sensitivity of the panel from the modal interaction between the global and local modes. PASCO tries to account for this interaction by allowing bow-type imperfections to be included during design. As discussed by Thompson and Hunt [16], an optimized design of an idealized perfect structure is imperfection sensitive, and only by including geometric nonlinearities and the coupling of buckling modes can this danger be avoided.

The interaction between the local and overall buckling modes was first discovered by Koiter [17], and later investigated by Graves-Smith [18] and van der Neut [19]. Many successful attempts to analyze the interaction with simplified models and to capture the interaction in experiments followed [1, 20-25] for stiffened isotropic structures. The the- 
ory of mode interaction was employed to study these problems. The displacements were written in a perturbation expansion in terms of an amplitude multiplier and a buckling mode shape function. These expansions were carried to the second-order such that mixed displacement effects between the buckling modes of interest and an understanding of these interactions were possible. A noticeable influence, termed "amplitude modulation", on the postbuckling behavior of stiffened panels was identified by Koiter [22]. The interaction of the global mode with the critical local mode was found to trigger two additional neighboring modes and these in turn triggered additional modes, and so on. Instead of including a larger number of modes in the analysis however, an account of the mode variation was made by allowing the amplitude of the local mode to vary according to a slowly varying function.

With the understanding that nonlinear effects must be included in the design of stiffened structures and the realization that composite stiffened panels possess significant postbuckling strength [26], attention shifted to developing approximate analyses that incorporated anisotropic materials, geometric nonlinearities, and modal interaction.

Sridharan and others [27-30] began developing a semi-analytical approach using the theory of mode interaction discussed in the previous paragraph and the finite strip method, which solves for local transverse displacements in one direction by discretizing the panel cross-section. The local buckling deformations are solved for by using a perturbation expansion. Amplitude modulation is taken into account by introducing slowly varying functions in the expansion of the local modes. The modulation of the amplitudes of the local modes and the overall displacements are described in terms of a one-dimensional finite element model. The theory was implemented in a computer program called INTBEAM.FOR [30] and incorporated into a strategy for the minimum-weight design of axi- 
ally compressed stiffened panels subject to mode interaction [31, 32]. The optimization technique used is based on Powell's algorithm (VMCON) [33]. Extension of this procedure into the area of composite materials is planned. This design approach was of particular interest to the author due to its similarity to the perturbation expansion of the local buckling modes.

Another preliminary design procedure, POSTOP (Postbuckled Open-Stiffener Optimum Panels), was developed at the Lockheed-Georgia Company [34-36] through the sponsorship of the NASA Langley Research Center. POSTOP minimizes the weight of symmetrically laminated composite panels with open cross-section stiffeners (for example, I-, T-, Zor J-stiffeners) loaded into the post-buckling range by a combination of inplane biaxial compression, or tension, or shear. Initial bow-type eccentricities are considered along with pressure and thermal effects. The approach taken to analyze the nonlinear response of the panel was to decompose the problem into separate postbuckling problems using beam-column theory [37]. The skin section is treated as a long simply-supported plate with restraints added along its long edges due to the stiffeners. The stiffeners are analyzed as an assembly of plate-strips to determine extensional, bending, torsional, and warping stiffnesses. The two possible stiffener local modes investigated include torsional modes, where the cross-section distorts, and rolling modes, where the cross-section translates. The skin section is allowed to reach a postbuckled state, however, the local buckling of the stiffener is treated as a failure mode. Material failure, based on maximum strain or the Tsai-Hill criterion, is checked in the skin and a simplified approach is used to minimize skin/stiffener separation. Design variables include stiffener plate widths, stiffener spacing, and ply thicknesses. The optimizer used in the design procedure is CONMIN [10]. 
Another preliminary design procedure based on the philosophy of using several separate analyses to capture the different nonlinear behaviors is PANDA2 [38,39]. The work of Bushnell over the years has led to this code which can find the minimum-weight designs of laminated composite flat or curved cylindrical panels or cylindrical shells with stiffeners in one or two orthogonal directions. The panel can have blade-, T-, J-, hat- or corrugated-stiffeners. The loadings include inplane biaxial and shear loads, edge moments, normal pressure, and temperature. Initial bow-type eccentricities as well as local imperfections in the form of the local buckling mode are considered. The types of buckling investigated separately include global buckling, local buckling of stiffeners, rolling buckling modes of stiffeners, torsional buckling modes of stiffeners, and crippling of stiffener segments. The panel may be allowed to develop local buckling, but is restrained from buckling in the other modes mentioned. There are also constraints placed on maximum displacement under pressure and material failure. The possible jump in axial wavelength of the local buckles is allowed, a phenomenon encountered as the panel is loaded further into the postbuckling region. The possible pop-off of the stiffener from the skin is accomodated through a user-supplied web peel-off force. The optimizer CONMIN [10] is used in the design procedure. 


\subsection{Analysis}

Key features of the nonlinear analysis approach developed in [4] are summarized here. Then, the dependence of the nonlinear analysis on selected buckling eigenfunction sets is investigated through parametric studies. The chapter ends with a discussion of the effects of initial imperfections on the nonlinear analysis and nonlinear design.

\subsection{Nonlinear Panel Analysis (NLPAN)}

The nonlinear analysis method developed in [4] uses a perturbation approach based on the use of a series of buckling eigenfunctions to represent displacement contributions associated with the nonlinear response. The buckling eigenfunctions are obtained using the linear buckling analysis code VIPASA contained within the buckling design code PASCO. Displacement contributions which are of second order in the modal amplitudes are incorporated in addition to the buckling eigenfunctions. Using a finite basis of buckling eigenfunctions, the stationary total potential energy condition is imposed and terms through the third order in the modal amplitudes are retained. A set of cubic nonlinear algebraic equations are obtained, from which approximate equilibrium solutions are determined. This section begins with a discussion of the nonlinear plate theory which governs the equilibrium of each component plate within the linked-plate structure (see Figure 2). Next, the characteristics of a general linked-plate structure are discussed, followed by the resulting governing nonlinear equations and method of solution. Additional information on and 
development of the NLPAN analysis can be found in References [4] and [40].

\subsubsection{Nonlinear Plate Theory}

2.1.1.1 Strain-Displacement Relations: Consider an undeformed thin plate with length $L$ in the $x$-direction and width $b$ in the $y$-direction in a Cartesian coordinate system $(x, y, z)$ (see Figure 3). The midplane of the plate, the $x-y$ plane, is the reference surface. Displacements of the midplane corresponding to the $x$-, $y$-, and $z$-directions are denoted by $u^{c}(x, y), v^{c}(x, y), w^{c}(x, y)$, respectively.

For thin plates, from the Kirchhoff assumptions, straight lines normal to the midplane in the undeformed plate remain straight and normal to the midplane after the plate is extended and bent. In addition, the line remains inextensional. Thus, inplane displacements $u, v$ and the transverse deflection $w$ at an arbitrary point of the plate may be approximated by

$$
\begin{gathered}
u(x, y, z)=u^{c}(x, y)-z \frac{\partial w^{c}}{\partial x}, \\
v(x, y, z)=v^{c}(x, y)-z \frac{\partial w^{c}}{\partial y}, \\
w(x, y, z)=w^{c}(x, y) .
\end{gathered}
$$

From the Kirchhoff assumption dealing with displacements, the following assumptions regarding the out-of-plane strains result: $\varepsilon_{z}=0$ (normals do not extend) and $\gamma_{x z}=\gamma_{y z}=0$ (no angular distortion between normals and the midplane). The inplane strain components, $\{\varepsilon\}=\left\{\varepsilon_{x}, \varepsilon_{y}, \gamma_{x y}\right\}$, are restricted to be small compared to unity, but rotation amplitudes are permitted to be moderately large. For a Lagrangian description of deformations (describing particles with respect to the original or undeformed configuration), the finite-strain expressions for the inplane strain components are given by [41] 


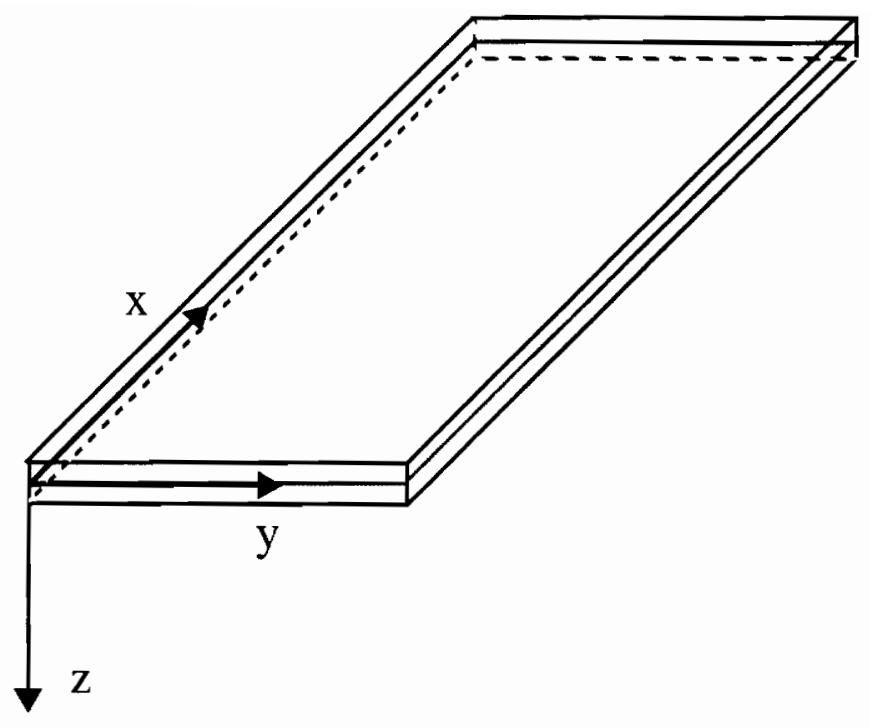

FIGURE 3. Convention of coordinate system. 


$$
\begin{aligned}
& \varepsilon_{x}=\frac{\partial u}{\partial x}+\frac{1}{2}\left[\left(\frac{\partial u}{\partial x}\right)^{2}+\left(\frac{\partial v}{\partial x}\right)^{2}+\left(\frac{\partial w}{\partial x}\right)^{2}\right] \\
& \varepsilon_{y}=\frac{\partial v}{\partial y}+\frac{1}{2}\left[\left(\frac{\partial u}{\partial y}\right)^{2}+\left(\frac{\partial v}{\partial y}\right)^{2}+\left(\frac{\partial w}{\partial y}\right)^{2}\right], \\
& \gamma_{x y}=\frac{\partial u}{\partial y}+\frac{\partial v}{\partial x}+\frac{\partial u \partial u}{\partial x} \frac{\partial v \partial v}{\partial x}+\frac{\partial w}{\partial y} \frac{\partial w}{\partial y}
\end{aligned}
$$

In the von Kármán nonlinear plate theory [41], the rotation about the $z$-axis is generally much smaller than those about the $x$ - or $y$-axis; thus, only displacement gradients $(\partial w / \partial x)$ and $(\partial w / \partial y)$ are of concern, leaving the nonlinear terms of the displacement gradients in $u$ and $v$ neglected. However, for linked-plate structures, gradients of $u$ and $v$ may become significantly large due to inplane rotation of a component plate, such as the rotation of a stiffener during global buckling of a panel. For this reason, the term $(\partial \nu / \partial x)^{2}$ in the expression for $\varepsilon_{x}$ and $(\partial u / \partial y)^{2}$ in the expression for $\varepsilon_{y}$ are retained to give the following expressions for the inplane strains:

$$
\begin{gathered}
\varepsilon_{x}=\frac{\partial u}{\partial x}+\frac{1}{2}\left[\left(\frac{\partial v}{\partial x}\right)^{2}+\left(\frac{\partial w}{\partial x}\right)^{2}\right], \\
\varepsilon_{y}=\frac{\partial v}{\partial y}+\frac{1}{2}\left[\left(\frac{\partial u}{\partial y}\right)^{2}+\left(\frac{\partial w}{\partial y}\right)^{2}\right], \\
\gamma_{x y}=\frac{\partial u}{\partial y}+\frac{\partial v}{\partial x}+\frac{\partial w}{\partial x} \frac{\partial w}{\partial y}
\end{gathered}
$$

These two additional terms, $(\partial v / \partial x)^{2}$ and $(\partial u / \partial y)^{2}$, do not appear in the von Kármán nonlinear strain expressions. Substitution of equations (1) into (3) gives the resulting expressions: 


$$
\begin{gathered}
\varepsilon_{x}=\frac{\partial u^{c}}{\partial x}+\frac{1}{2}\left[\left(\frac{\partial v^{c}}{\partial x}\right)^{2}+\left(\frac{\partial w^{c}}{\partial x}\right)^{2}\right]-z\left(\frac{\partial^{2} w^{c}}{\partial x^{2}}\right) \\
\varepsilon_{y}=\frac{\partial v^{c}}{\partial y}+\frac{1}{2}\left[\left(\frac{\partial u^{c}}{\partial y}\right)^{2}+\left(\frac{\partial w^{c}}{\partial y}\right)^{2}\right]-z\left(\frac{\partial^{2} w^{c}}{\partial y^{2}}\right) \\
\gamma_{x y}=\frac{\partial u^{c}}{\partial y}+\frac{\partial v^{c}}{\partial x}+\frac{\partial w^{c}}{\partial x} \frac{\partial w^{c}}{\partial y}-2 z \frac{\partial^{2} w^{c}}{\partial x \partial y}
\end{gathered}
$$

where higher order terms involving derivatives and terms nonlinear in $z$ have been neglected. The resulting $z$-dependence is the same as that of classical linear plate theory. Equations (4) in terms of midplane strains, $\left\{\varepsilon^{c}\right\}$, and curvatures, $\left\{\kappa^{c}\right\}$, are given by

$$
\begin{gathered}
\varepsilon_{x}(x, y, z)=\varepsilon_{x}^{c}(x, y)+z \kappa_{x}^{c}(x, y), \\
\varepsilon_{y}(x, y, z)=\varepsilon_{y}^{c}(x, y)+z \kappa_{y}^{c}(x, y), \\
\gamma_{x y}(x, y, z)=\gamma_{x y}^{c}(x, y)+z \kappa_{x y}^{c}(x, y),
\end{gathered}
$$

where

$$
\begin{gathered}
\varepsilon_{x}^{c}=\frac{\partial u^{c}}{\partial x}+\frac{1}{2}\left[\left(\frac{\partial v^{c}}{\partial x}\right)^{2}+\left(\frac{\partial w^{c}}{\partial x}\right)^{2}\right], \quad \varepsilon_{y}^{c}=\frac{\partial v^{c}}{\partial y}+\frac{1}{2}\left[\left(\frac{\partial u^{c}}{\partial y}\right)^{2}+\left(\frac{\partial w^{c}}{\partial y}\right)^{2}\right], \\
\gamma_{x y}^{c}=\frac{\partial u^{c}}{\partial y}+\frac{\partial v^{c}}{\partial x}+\frac{\partial w^{c}}{\partial x} \frac{\partial w^{c}}{\partial y}, \\
\kappa_{x}^{c}=-\frac{\partial^{2} w^{c}}{\partial x^{2}}, \quad \kappa_{y}^{c}=-\frac{\partial^{2} w^{c}}{\partial y^{2}}, \quad \kappa_{x y}^{c}=-2 \frac{\partial^{2} w^{c}}{\partial x \partial y} .
\end{gathered}
$$

Geometric imperfections are accounted for by assuming, when the panel is free from load, that the displacements $\left\{u^{o}\right\}=\left\{u^{o}, v^{o}, w^{o}\right\}$ describe the imperfection shape. Thus the mechanical midplane strains, $\left\{\varepsilon^{m}\right\}$, and curvatures, $\left\{\kappa^{m}\right\}$, are given by 


$$
\begin{gathered}
\varepsilon_{x}^{m}=\left[\frac{\partial u}{\partial x}+\frac{1}{2}\left(\left(\frac{\partial v}{\partial x}\right)^{2}+\left(\frac{\partial w}{\partial x}\right)^{2}\right)\right]-\left[\left(\frac{\partial u^{o}}{\partial x}+\frac{1}{2}\left(\left(\frac{\partial v^{o}}{\partial x}\right)^{2}+\left(\frac{\partial w^{o}}{\partial x}\right)^{2}\right)\right)\right] \\
\varepsilon_{y}^{m}=\left[\frac{\partial v}{\partial y}+\frac{1}{2}\left(\left(\frac{\partial u}{\partial y}\right)^{2}+\left(\frac{\partial w}{\partial y}\right)^{2}\right)\right]-\left[\left(\frac{\partial v^{o}}{\partial y}+\frac{1}{2}\left(\left(\frac{\partial u^{o}}{\partial y}\right)^{2}+\left(\frac{\partial w^{o}}{\partial y}\right)^{2}\right)\right)\right] \\
\gamma_{x y}^{m}=\left[\frac{\partial u}{\partial y}+\frac{\partial v}{\partial x}+\frac{\partial w}{\partial x} \frac{\partial w}{\partial y}\right]-\left[\frac{\partial u^{o}}{\partial y}+\frac{\partial v^{o}}{\partial x}+\frac{\partial w^{o}}{\partial x} \frac{\partial w^{o}}{\partial y}\right] \\
\kappa_{x}^{m}=-\left[\frac{\partial^{2} w}{\partial x^{2}}-\frac{\partial^{2} w^{o}}{\partial x^{2}}\right] \\
\kappa_{y}^{m}=-\left[\frac{\partial^{2} w}{\partial y^{2}}-\frac{\partial^{2} w^{o}}{\partial y^{2}}\right] \\
\kappa_{x y}^{m}=-\left[2 \frac{\partial^{2} w}{\partial x \partial y}-2 \frac{\partial^{2} w^{o}}{\partial x \partial y}\right]
\end{gathered}
$$

where the subscript $c$ has been dropped from the midplane displacements for convenience.

2.1.1.2 Equilibrium Equations: All loads are applied at the plate edges. The thin plate is assumed to be in a state of plane stress, thus the transverse shear stresses $\sigma_{x z}$ and $\sigma_{y z}$ are neglected, consistent with the Kirchhoff assumptions, and the transverse normal stress $\sigma_{z}$ is neglected. The Euler equations, resulting from the principle of virtual work, are

$$
\begin{aligned}
& \frac{\partial N}{\partial x}^{x}+\frac{\partial N}{\partial y}+\frac{\partial}{\partial y}\left(N_{y} \frac{\partial u}{\partial y}\right)=0 \\
& \bar{\partial}_{x y}+\frac{\partial N_{y}}{\partial y}+\frac{\partial}{\partial x}\left(N_{x} \frac{\partial v}{\partial x}\right)=0 \\
& \frac{\partial^{2} M_{x}}{\partial x^{2}}+2 \frac{\partial^{2} M_{x y}}{\partial x \partial y}+\frac{\partial^{2} M_{y}}{\partial y^{2}}+\frac{\partial}{\partial x}\left(N_{x} \frac{\partial w}{\partial x}+N_{x y} \frac{\partial w}{\partial y}\right)+\frac{\partial}{\partial y}\left(N_{x y} \frac{\partial w}{\partial x}+N_{y} \frac{\partial w}{\partial y}\right)=0
\end{aligned}
$$

where the stress resultants are defined by 


$$
\left\{\begin{array}{c}
N_{x} \\
N_{y} \\
N_{x y}
\end{array}\right\}=\int_{-\frac{h}{2}}^{\frac{h}{2}}\left\{\begin{array}{c}
\sigma_{x} \\
\sigma_{y} \\
\sigma_{x y}
\end{array}\right\} d z, \quad\left\{\begin{array}{c}
M_{x} \\
M_{y} \\
M_{x y}
\end{array}\right\}=\int_{-\frac{h}{2}}^{\frac{h}{2}}\left\{\begin{array}{c}
\sigma_{x} \\
\sigma_{y} \\
\sigma_{x y}
\end{array}\right\} z d z
$$

where $h$ is the plate thickness.

2.1.1.3 Constitutive Relations: For this study, the plates within a panel are composed of linear elastic material, following Hooke's Law. The elastic properties of each plate are assumed to be those of a balanced, symmetric laminated composite plate, thus the extension-bending coupling stiffness matrix $[B]$ and the extension-shearing stiffness terms $A_{16}$ and $A_{26}$ are zero. In addition, the bending-twisting stiffness terms $D_{16}$ and $D_{26}$ are neglected. The uncoupled relations between the force resultants $\{N\}$ and the midplane mechanical strains $\left\{\varepsilon^{m}\right\}$ and the moment resultants $\{M\}$ and the midplane mechanical curvatures $\left\{\kappa^{m}\right\}$ are

$$
\left\{\begin{array}{c}
N_{x} \\
N_{y} \\
N_{x y}
\end{array}\right\}=\left[\begin{array}{ccc}
A_{11} & A_{12} & 0 \\
A_{12} A_{22} & 0 \\
0 & 0 & A_{66}
\end{array}\right]\left\{\begin{array}{c}
\varepsilon_{x}^{m} \\
\varepsilon_{y}^{m} \\
\gamma_{x y}^{m}
\end{array}\right\}, \quad\left\{\begin{array}{l}
M_{x} \\
M_{y} \\
M_{x y}
\end{array}\right\}=\left[\begin{array}{ccc}
D_{11} & D_{12} & 0 \\
D_{12} & D_{22} & 0 \\
0 & 0 & D_{66}
\end{array}\right]\left\{\begin{array}{c}
\kappa_{x}^{m} \\
\kappa_{y}^{m} \\
\kappa_{x y}^{m}
\end{array}\right\} .
$$

The extensional and bending stiffness terms above follow laminated plate theory definitions given by, for example, Jones [42].

\subsubsection{Linked-Plate Geometry}

2.1.2.1 Geometry: The stiffened panel configurations being studied are composed of thin rectangular plates linked along their longitudinal sides (see Figure 4). The panel has an associated global coordinate system, while the individual plates have associated local coordinate systems. The global $X$-axis is along the length of the panel and is in the same 
direction as the local $x$-axes for the individual plates. The global $Y$-axis points along the widths, $b$, of the plate strips within the skin of the panel. The global $Z$-axis points in the direction such that a positive displacement is one towards the stiffener. The local coordinate system of each plate is such that the $y$-axis is along the width of the plate segment and the local $z$-axis direction follows the right-hand rule. The panel is built by numbering each plate and the node lines where two plates are connected. In the end, there can be a number of internal nodes lines (not to be confused with node lines of a buckling mode shape), however there are only two boundary node lines in which the boundary conditions and possible external loadings are applied. The coordinates and numbering system are illustrated in Figure 4 for the blade-stiffened panel analyzed in later sections (see sections 2.2, 2.3, and 4.1).

\subsubsection{Boundary Conditions and Loading: Along the panel ends $(X=0, L)$, the} boundary conditions are restricted to be simply-supported due to the assumed displacements within VIPASA. Along the external boundary nodes $(Y=0, B)$, a variety of boundary conditions, including free, simple support, symmetry, and clamped, can be enforced. The inplane loading may be either uniaxial or biaxial. The generalized inplane load parameter is designated as $\lambda$ and can be used to control either edge displacements or edge forces. For this study, the loading is uniaxial and edge displacement control is used. The options available for controlling the inplane loading are summarized in Table 1.

The parameters $N_{x G}$ and $N_{y G}$ appearing in Table 1 are the mean values of $N_{x}$ and $N_{y}$, respectively, acting along an $X$-normal and $Y$-normal edge, respectively. Parameters with the subscript $L$ represent the linear, unbuckled response associated with a unit inplane load system. This unit solution is discussed in a later section 2.1.3.1.

The simple support condition along the $X$-normal edges $(X=0, L)$ has the following characteristics in addition to those chosen in Table 1: 


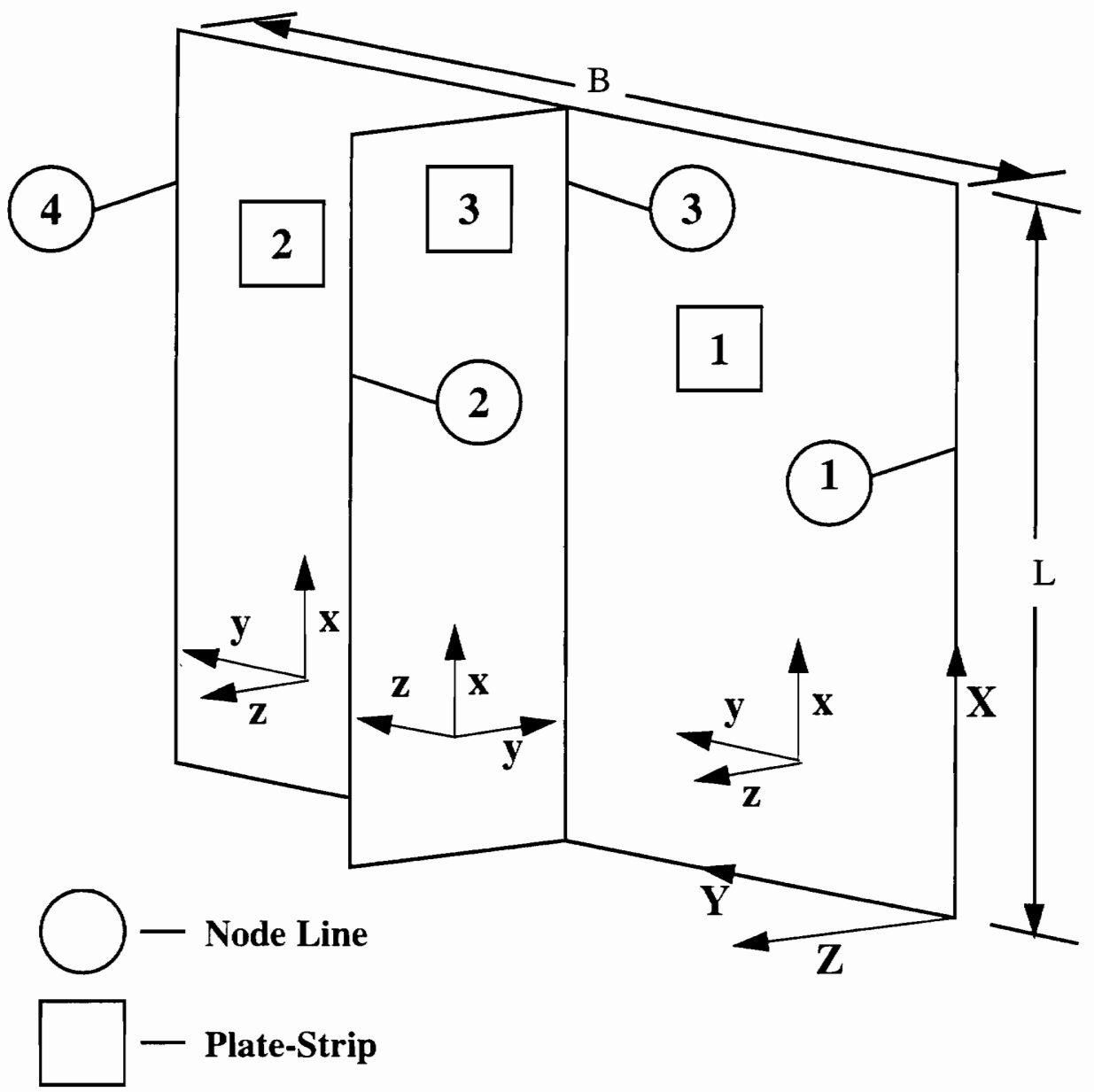

FIGURE 4. Stiffened panel illustrating plate-strip numbers, node line numbers, and labeling conventions: Note that the local plate-strip axes shown do not reflect actual locations of origin. [4] 
Table 1: Options for Controlling the Inplane Loading. [4]

\begin{tabular}{|c|c|c|c|}
\hline Edge & Option & $\begin{array}{c}\text { Displacement } \\
\text { Control }\end{array}$ & Load Control \\
\hline \hline $\mathrm{X}=0, \mathrm{~L}$ & & $\begin{array}{c}u=\lambda u_{L} \\
\left(\Delta u=\lambda \Delta u_{L}\right)\end{array}$ & $\left(\frac{1}{B}\right) \int_{0}^{B} \hat{N}_{x} d y=N_{x G}=\lambda N_{x L}$ \\
\hline $\mathrm{Y}=0, \mathrm{~B}$ & 1 & $\begin{array}{c}v=\lambda v_{L} \\
\left(\Delta v=\lambda \Delta v_{L}\right)\end{array}$ & $v=0$ \\
\hline & 2 & $N_{y}=0$ & $\hat{N}_{y}=\lambda N_{y L}$ \\
\hline & 3 & $\frac{\partial v}{\partial x}=0$ & $\frac{\partial v}{\partial x}=0$ \\
& & $\int_{0}^{L} N_{y} d x=0$ & $\left(\frac{1}{L}\right) \int_{0}^{L} \hat{N}_{y} d x=N_{y G}=\lambda N_{y L}$ \\
\hline
\end{tabular}

$$
\begin{gathered}
N_{x y}=0, \\
w=0, \\
M_{x}=0 .
\end{gathered}
$$

Along each of the external $Y$-normal edges $(Y=0, B)$, in addition to those chosen in Table 1, the free, simple support, symmetry, and clamped boundary conditions can be modelled by selecting from the following options:

$$
\begin{aligned}
u=0 & \text { or } & f_{x}=0, \\
w=0 & \text { or } & f_{z}=0, \\
\frac{\partial w}{\partial y}=0 & \text { or } & M_{y}=0,
\end{aligned}
$$

where the edge force-resultants (force per unit length) $f_{x}$ and $f_{z}$ given by 


$$
\begin{gathered}
f_{x}=n_{y}\left(N_{x y}+N_{y} \partial u / \partial y\right) \\
f_{z}=n_{y}\left(2 \partial M_{x y} / \partial x+\partial M_{y} / \partial y+N_{x y} \partial w / \partial x+N_{y} \partial w / \partial y\right) .
\end{gathered}
$$

Parameter $n_{y}$ is the $y$-component of the unit vector normal to a plate edge and takes the values of \pm 1 depending on whether the right or the left edge is being represented.

The primary global load component applied to the panel is the mean $X$-normal force per unit width, $N_{x G}$, defined as the total load divided by the reference width $B$. A transverse nonzero global load component, $N_{y G}$, may also be applied to plate edges representing the skin of the panel featuring a continuous, initially flat skin. Component $N_{y G}$ is the mean force per unit length (based on length $L$ ) acting in the global $Y$ direction. For this study, an analysis is initiated by specifying a unit load $\hat{N}_{x G}$ or a unit end shortening $\Delta \hat{u}$.

\subsubsection{Expansions of Displacement Functions}

The displacements have the following assumed form on each plate strip:

$$
\{u\}=\lambda\left\{u_{L}\right\}+q_{i}\left\{u_{i}\right\}+q_{i} q_{j}\left\{u_{i j}\right\}, \quad i, j=1,2,3, \ldots
$$

where summation over $i$ and $j$ is implied, and the notation $\{u\}=\{u, v, w\}$ is used to refer to a set of compatible displacement fields. Displacements are represented as the sum of linear, unbuckled contributions, $\left\{u_{L}\right\}$, and a truncated perturbation expansion in terms of modal amplitudes, $q_{i}$, which are the unknown amplitude multipliers for the buckling eigenfunctions, $\left\{u_{i}\right\}$. The magnitudes of $q_{i}$ determine the amount of influence their respective buckling modes have on the response of the panel. Displacement contributions of second order in the modal amplitudes are retained, $\left\{u_{i j}\right\}$. These contributions are deemed important in order to achieve solutions of useful accuracy for a variety of geometric configurations. In equation (14), $\lambda$ is the load parameter controlling end-shortening. For an unloaded panel $(\lambda=0)$, the displacements degenerate to the imperfection shape of 
the panel:

$$
\left\{u^{o}\right\}=q_{i}^{o}\left\{u_{i}\right\}+q_{i}^{o} q_{j}^{o}\left\{u_{i j}\right\}, \quad i, j=1,2,3, \ldots
$$

where $q_{i}^{o}$ are the modal imperfection amplitudes that generate imperfections in the shape of their respective buckling modes.

The assumed form for the displacements and the imperfection shapes are used in the expressions for the midplane mechanical strains and curvatures of equation (7). Using these expansions along with the plate constitutive equations (10), the stress resultants are similarly expanded. In order to obtain the equations which govern the displacement shape functions that appear in equation (14), the plate equilibrium equations (8) are expressed using the expanded forms of the displacements and stress resultants. For the case in which the imperfection amplitudes are zero the following equations are obtained: 


$$
\begin{aligned}
& q_{i}\left[\frac{\partial N_{x_{i}}}{\partial x}+\frac{\partial N}{\partial y}^{x y_{i}}+\lambda N_{y L} \frac{\partial^{2} u_{i}}{\partial y^{2}}\right]+ \\
& q_{i} q_{j}\left[\frac{\partial N_{x_{i j}}}{\partial x}+\frac{\partial N_{x y_{i j}}}{\partial y}+\frac{\partial}{\partial y}\left(N_{y_{j}} \frac{\partial u_{i}}{\partial y}\right)+\lambda N_{y L} \frac{\partial^{2} u_{i j}}{\partial y^{2}}\right]+O\left(q_{i}^{3}\right)=0, \\
& q_{i}\left[\overline{\partial N}_{x y_{i}}+{\frac{\partial N_{y_{i}}}{\partial y}}+\lambda N_{x L} \frac{\partial^{2} v_{i}}{\partial x^{2}}\right]+ \\
& q_{i} q_{j}\left[\frac{\partial N_{x y_{i j}}}{\partial x}+\frac{\partial N_{y_{i j}}}{\partial y}+\frac{\partial}{\partial x}\left(N_{x} \frac{\partial v_{i}}{\partial x}\right)+\lambda N_{x L} \frac{\partial^{2} v_{i j}}{\partial x^{2}}\right]+O\left(q_{i}^{3}\right)=0, \\
& q_{i}\left[\frac{\partial^{2} M_{x_{i}}}{\partial x^{2}}+2 \frac{\partial^{2} M_{x y_{i}}}{\partial x \partial y}+\frac{\partial^{2} M_{y_{i}}}{\partial y^{2}}+\lambda\left(N_{x L} \frac{\partial^{2} w_{i}}{\partial x^{2}}+N_{y L} \frac{\partial^{2} w_{i}}{\partial y^{2}}\right)\right]+
\end{aligned}
$$

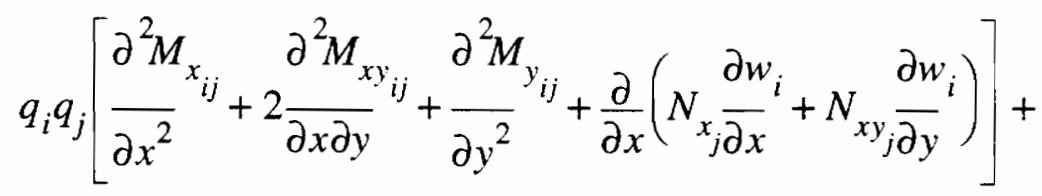

$$
\begin{aligned}
& q_{i} q_{j}\left[\frac{\partial}{\partial y}\left(N_{x y} \frac{\partial w_{i}}{\partial x}+N_{y} \frac{\partial w_{i}}{\partial y}\right)+\lambda\left(N_{x L} \frac{\partial^{2} w_{i j}}{\partial x^{2}}+N_{y L} \frac{\partial^{2} w_{i j}}{\partial y^{2}}\right)\right]+O\left(q_{i}^{3}\right)=0
\end{aligned}
$$

Bracketed expressions with a common order in modal amplitudes, $q_{i}$ in the above expansions set the grounds for determination of the buckling eigensolutions and the secondorder displacement fields. In the following three sections, details are given for the methods of determining the linear unbuckled solutions, the buckling eigensolutions, and the second-order displacement fields.

2.1.3.1 Linear Solutions: The displacement shape function associated with the linear unbuckled response of the perfect structure $\left\{u_{L}\right\}=\left\{u_{L}, v_{L}, 0\right\}$ describes the response to the specified global unit loads $\hat{N}_{x G}$ and $\hat{N}_{y G}$, where the longitudinal strain $\varepsilon_{x}$ is 
required to be uniform throughout the structure, ensuring compatibility of displacements at plate-strip junctions in the $X$-direction. For the stiffened panels in this study, only an axial end load is applied. Thus, the solution of the prebuckling strains and stress resultants is as follows. The global end load is related to the unit end load by

$$
N_{x G}=\lambda \hat{N}_{x G}
$$

With a specified unit end loading, the unit strains are calculated by

$$
\begin{gathered}
\hat{\varepsilon}_{x L}=\frac{\hat{N}_{x G} B}{\sum_{p=1}^{P} b_{p}\left(A_{11}-\frac{A_{12}^{2}}{A_{22}}\right)_{p}}, \\
\hat{\varepsilon}_{y L}=-\frac{\hat{\varepsilon}_{x L} A_{12}^{p}}{A_{22}^{p}},
\end{gathered}
$$

where there is a summation over the plate-strips, $p$, for a total of $P$ plate-strips. The unit stress resultant within each plate strip is then calculated by

$$
\hat{N}_{x L}^{p}=A_{11}^{p} \hat{\varepsilon}_{x L}+A_{12}^{p} \hat{\varepsilon}_{y L}^{p}
$$

2.1.3.2 Buckling Eigensolutions: The equations which determine the buckling eigenfunctions are obtained by setting to zero the terms of the expanded equilibrium equations (16) which are of first order in the modal amplitudes: 


$$
\begin{aligned}
& {\frac{\partial N_{x_{i}}}{\partial x}}+{\frac{\partial N_{x y_{i}}}{\partial y}}+\lambda_{i} N_{y L} \frac{\partial^{2} u_{i}}{\partial y^{2}}=0, \\
& \overline{\partial x}_{x y_{i}}+\frac{\partial N_{y_{i}}}{\partial y}+\lambda_{i} N_{x L} \frac{\partial^{2} v_{i}}{\partial x^{2}}=0,
\end{aligned}
$$

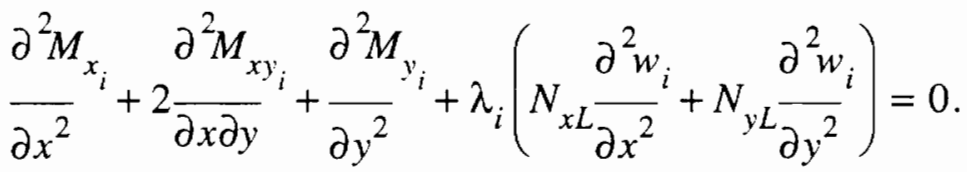

The subscript $i$ is for each eigenfunction chosen to be included in the solution process with $\lambda_{i}$ representing the corresponding eigenvalue.

The above information is calculated by the VIPASA analysis within PASCO. VIPASA is capable of determining the primary buckling eigenvalue for a given longitudinal halfwavelength number, and any desired number of additional eigenvalues at that half-wavelength number. The $i^{\text {th }}$ eigenfunction has the following assumed form on each plate strip (where a phase shift in the $x$-direction has been applied relative to the conventions of VIPASA in order to locate the $x$-domain of the panel in the interval $[0, L])$ :

$$
\left\{u_{i}\right\}=\left\{\begin{array}{c}
u_{i} \\
v_{i} \\
w_{i}
\end{array}\right\}=\left\{\begin{array}{l}
\xi_{i}(y) \cos \frac{m \pi x}{L} \\
\eta_{i}(y) \sin \frac{m \pi x}{L} \\
\phi_{i}(y) \sin \frac{m \pi x}{L}
\end{array}\right\},
$$

where $m$ is the number of half-wavelengths along the length of the panel for the $i^{\text {th }}$ eigensolutions. The $x$-dependent functions in the above equation show the restricted boundary conditions of simple support stated earlier. The $y$-dependent functions are not restricted and thus allow for a number of boundary conditions. These functions are determined by substituting equation (21) into equation (20). Details of the solution can be obtained in [4]. 
2.1.3.3 Second-Order Displacement Fields: The equations used to determine the functions $\left\{u_{i j}\right\}=\left\{u_{i j}, v_{i j}, w_{i j}\right\}$ for each plate strip are obtained by setting to zero the terms of the expanded equilibrium equations (16) which are of second order in the modal amplitudes:

$$
\begin{aligned}
& \overline{\partial x}_{x_{i j}}+\frac{\partial N_{x y_{i j}}}{\partial y}+\frac{1}{2}\left[\frac{\partial}{\partial y}\left(N_{y} \frac{\partial}{\partial y}\left(u_{j}\right)\right)+\frac{\partial}{\partial y}\left(N_{y_{j}} \frac{\partial u_{i}}{\partial y}\right)\right]=0, \\
& \bar{\partial}_{x x}{ }^{\partial j}+\bar{\partial}_{y_{i j}}+\lambda_{b} N_{x L} \frac{\partial^{2} v_{i j}}{\partial x^{2}}+\frac{1}{2}\left[\frac{\partial}{\partial x}\left(N_{x} \frac{\partial v_{j}}{\partial x}\right)+\frac{\partial}{\partial x}\left(N_{x} \frac{\partial v_{i}}{\partial x}\right)\right]=0,
\end{aligned}
$$

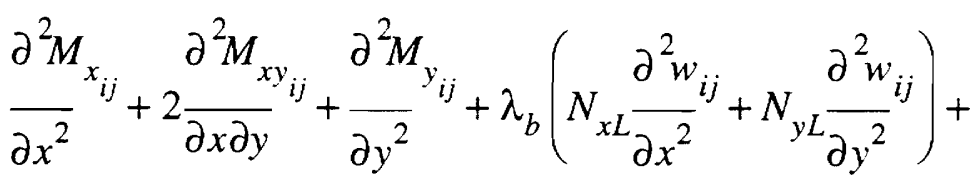

$$
\begin{aligned}
& \frac{1}{2}\left[\frac{\partial}{\partial x}\left(N_{x} \frac{\partial w_{j}}{\partial x}+N_{x y} \frac{\partial w_{j}}{\partial y}\right)+\frac{\partial}{\partial y}\left(N_{x y} \frac{\partial w_{j}}{\partial x}+N_{y_{i}} \frac{\partial w_{j}}{\partial y}\right)+\right. \\
& \left.\frac{\partial}{\partial x}\left(N_{x_{j}} \frac{\partial w_{i}}{\partial x}+N_{x y} \frac{\partial w_{i}}{\partial y}\right)+\frac{\partial}{\partial y}\left(N_{x y} \frac{\partial w_{i}}{\partial x}+N_{y_{j}} \frac{\partial w_{i}}{\partial y}\right)\right]=0 .
\end{aligned}
$$

The parameter $\lambda_{b}$ is a reference value of $\lambda$, not necessarily the exact value of $\lambda$ at which an equilibrium solution is sought. For this study, it is set to zero. The above equations involve known eigenfunctions $\left\{u_{i}\right\}$ and $\left\{u_{j}\right\}$, and the unknown shape functions $\left\{u_{i j}\right\}$. The number of half-wavelengths in the $x$-direction for eigenfunctions $\left\{u_{i}\right\}$ and $\left\{u_{j}\right\}$ are $m$ and $n$, respectively. The assumed form for $\left\{u_{i j}\right\}$ suggested by Sridharan [27]:

$$
\left\{u_{i j}\right\}=\sum_{\alpha=1}^{2}\left\{\begin{array}{l}
\xi_{\alpha_{i j}}(y) \sin \frac{\hat{m} \pi x}{L} \\
\eta_{\alpha_{i j}}(y) \cos \frac{\hat{m} \pi x}{L} \\
\phi_{\alpha_{i j}}(y) \cos \frac{\hat{m} \pi x}{L}
\end{array}\right\},
$$

where 


$$
\hat{m}=\left(\begin{array}{ll}
m+n & \alpha=1 \\
m-n & \alpha=2
\end{array}\right.
$$

Details of the solution of the second-order displacement fields are given in [4].

\section{$\underline{\text { 2.1.4 Solution of Equations }}$}

With the shape functions determined as described in the previous section, it remains to determine the final, approximate equilibrium solutions. The approach involves a direct application of the virtual work statement. A set of $M$ eigensolutions is selected to establish a finite basis for the expression of displacements, generalized strains, stress resultants, and so forth. The virtual work statement is evaluated with the expanded forms for the generalized strains and stress resultants which have been developed, and expressed in the following form:

$$
C_{i}^{o}+q_{j}\left(C_{i j}^{o}+\lambda C_{i j}^{L}\right)+q_{j} q_{k} q_{l} C_{i j k l}=0, \quad i=1,2, \ldots, M
$$

where

$$
\begin{gathered}
C_{i}^{o}=-q_{j} C_{i}^{j}, \\
C_{i j}^{o}=C_{i j}-q_{k}^{o} q_{l}^{o} C_{i j}^{k l} .
\end{gathered}
$$

The sub- and super-scripted coefficients $C$ are constants defined in equation (141) of [4]. Equation (25) governs the equilibrium behavior of a panel subjected to loads governed by the parameter $\lambda$. Solution methods which can navigate limit points and bifurcation points are used. The first method is the arc-length control method popularized by Riks [43] that is generally used to traverse limit points. For branching at simple or multiple bifurcation points, a method developed by Thurston [44] is used. A recent paper by Stoll [40] describes in detail these methods and their implementation into the NLPAN code. 


\subsection{Mode Selection}

As stated in section 2.1.3, the nonlinear analysis requires a set of buckling eigenfunctions provided by VIPASA to represent displacement contributions associated with the nonlinear response. The predetermination of a general set of eigenfunctions that yields an accurate representation of the postbuckling deformation is perhaps the biggest obstacle for automating this design tool.

With NLPAN in an analysis mode, the user has the option of reviewing the accuracy of any number of buckling mode sets before a final set is selected. Since only a single analysis is performed, the modal amplitudes for each buckling mode are available for review after every analysis. Thus, the amount of influence a particular mode has on the postbuckling response can be assessed by the magnitude of its modal amplitude and the mode set may be altered accordingly.

In a design mode, the user does not have the opportunity to analyze a configuration so thoroughly. Moreover, the complexity of modelling the nonlinear characteristic behavior is increased due to the nature of the design process that changes the configuration, thus changing the response of the panel. A set of eigenfunctions chosen to represent the deformations of an initial configuration may not be suitable to represent the deformations of the panel as it is modified by the optimizer. One solution may be to choose many buckling modes over a wide range of half-wavelengths; however, such an approach increases the time and cost of repetitive analyses. Each additional mode almost doubles the computing time for a single analysis. Since it is the computational efficiency of NLPAN that makes it advantageous for the role in this design procedure, a minimum number of modes is preferred. Therefore, a strategy has been established for the automatic selection of a small set of buckling eigensolutions. The steps taken to determine this strategy are now discussed. 
The strategy relies upon the selection of a set of eigenmodes which are observed to lead to an accurate analysis based on extensive numerical testing. Table 2 lists five buckling mode sets investigated. The buckling modes are designated by two numbers, the first standing for the number of half-wavelengths in the $X$-direction and the second standing for the particular eigensolution at that half-wavelength number. For example, the normalized eigenvalues of a panel under an axial compression of $N_{x}=1000 \mathrm{lb} /$ in are shown in Figure 5 as a function of the buckling half-wavelength number. The three points at each half-wavelength number correspond to three distinct eigenvalues with the same number of halfwavelengths along the panel length but with different shapes in the transverse direction. The first eigenvalue is referred to as the primary value for that half-wavelength number and any additional eigenvalues at that half-wavelength number are referred to as secondary values. An asterisk is used in Table 2 to designate a secondary mode that has a similar symmetry feature as that of the primary mode at a particular half-wavelength number. In other words, the secondary mode of interest is determined such that its cross-sectional shape has the same symmetry features as the primary mode. For example, for a half-wavelength number of eight (which is the critical local half-wavelength number for this example), the cross-sectional buckling mode shapes for the three eigenvalues in Figure 5 are plotted at the panel midlength in Figure 6 . The antisymmetry of the first mode must be matched in choosing the secondary mode at this half-wavelength number, thus the third eigenvalue would be chosen. The symmetry of the secondary mode is important for representing possible nonlinear displacements by the addition or subtraction of the secondary displacements with the primary mode. For example, if the second mode was chosen in Figure 6, no additional blade deformations would be added to the analysis.

This research deals with geometries that generally exhibit only two principal modes of buckling: a local mode involving the thin plate elements between the stiffeners, and an 


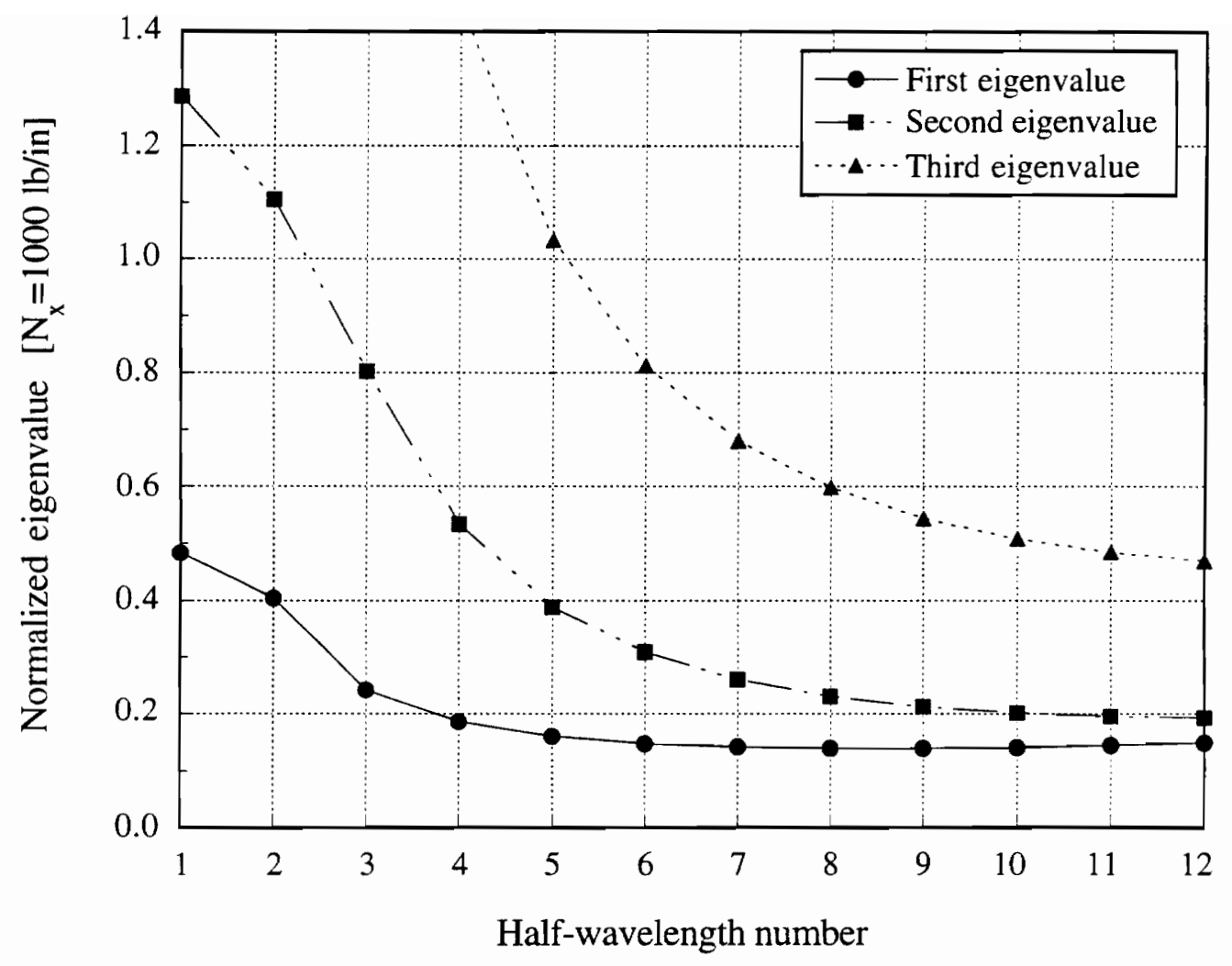

FIGURE 5. Example normalized eigenvalues scanned for selection in NLPANOPT mode sets. 

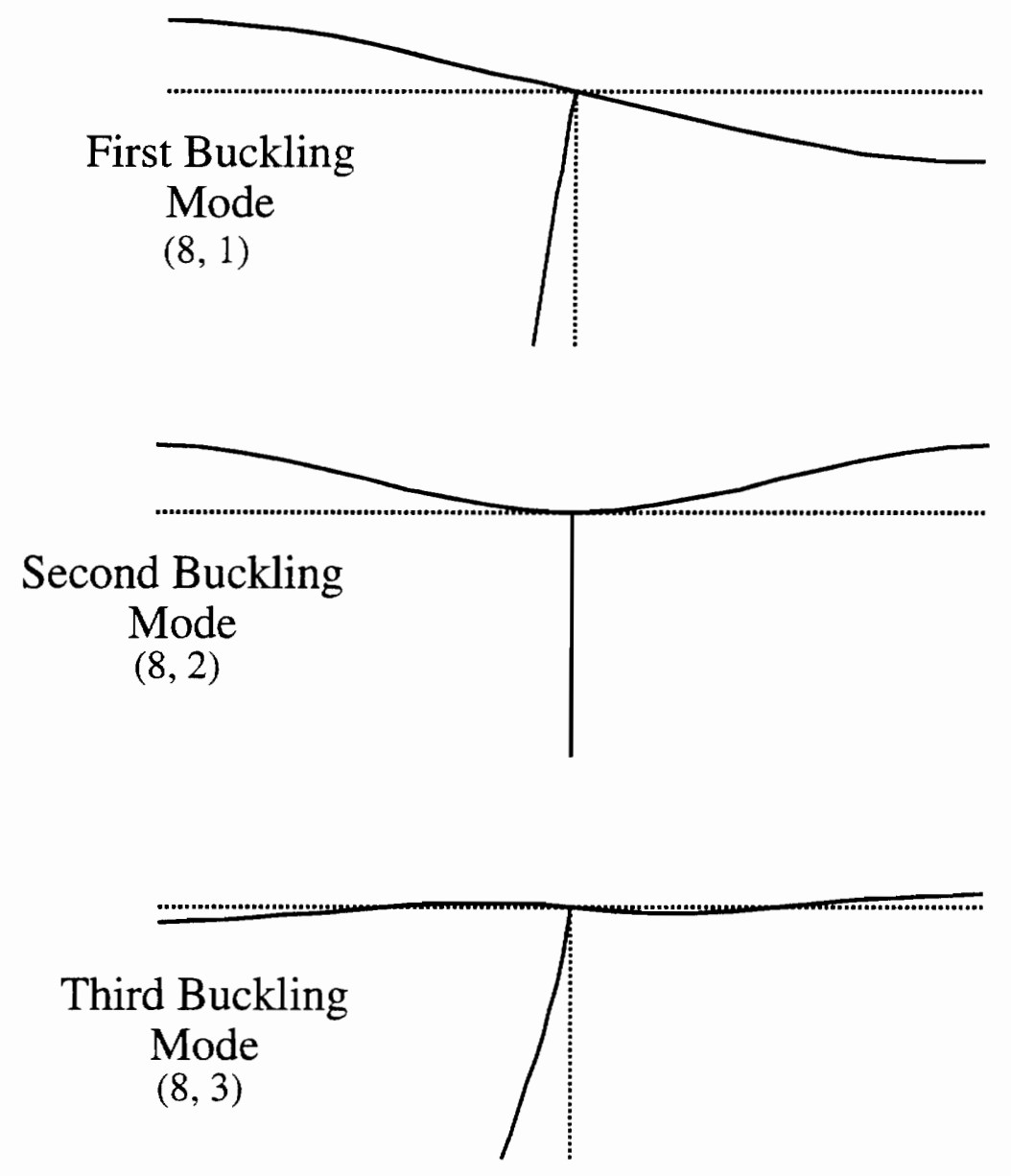

FIGURE 6. Cross-sectional buckling mode shapes at panel midlength for half-wavelength number 8 for example eigenvalues plotted in Figure 5. 
overall or global mode. Thus, the minimum buckling mode set needed is the one denoted Mode Set I in Table2 which includes:

1. a global buckling mode with one half-wavelength along the panel length,

2. a critical local buckling mode with a half-wavelength number denoted by $\mathrm{m}_{\mathrm{cr}}$,

3. a secondary local buckling mode that has the same $\mathrm{m}_{\mathrm{cr}}$ as the critical local buckling mode with a similar symmetry. This mode is included because it has been found to refine the nonlinear deformations in the postbuckling region [4].

Table 2: Summary of Mode Sets ( $\mathrm{X}$ indicates mode included, * indicates number of first secondary mode having the same symmetry as the primary mode)

\begin{tabular}{|c|c|c|c|c|c|}
\hline \multirow{2}{*}{$\begin{array}{c}\text { Buckling } \\
\text { Mode }\end{array}$} & \multicolumn{5}{|c|}{ Mode Set } \\
\cline { 2 - 6 } & $\mathrm{I}$ & $\mathrm{II}$ & $\mathrm{III}$ & $\mathrm{IV}$ & $\mathrm{V}$ \\
\hline \hline$(1,1)$ & $\mathrm{X}$ & $\mathrm{X}$ & $\mathrm{X}$ & $\mathrm{X}$ & $\mathrm{X}$ \\
\hline$(1,2)$ & & $\mathrm{X}$ & $\mathrm{X}$ & $\mathrm{X}$ & $\mathrm{X}$ \\
\hline$\left(\mathrm{m}_{\mathrm{cr}}, 1\right)$ & $\mathrm{X}$ & $\mathrm{X}$ & $\mathrm{X}$ & $\mathrm{X}$ & $\mathrm{X}$ \\
\hline$\left(\mathrm{m}_{\mathrm{cr}}{ }^{*}\right)$ & $\mathrm{X}$ & $\mathrm{X}$ & $\mathrm{X}$ & $\mathrm{X}$ & $\mathrm{X}$ \\
\hline$\left(\mathrm{m}_{\mathrm{cr}}-2,1\right)$ & & & & $\mathrm{X}$ & $\mathrm{X}$ \\
\hline$\left(\mathrm{m}_{\mathrm{cr}}-2,{ }^{*}\right)$ & & & & $\mathrm{X}$ & $\mathrm{X}$ \\
\hline$\left(\mathrm{m}_{\mathrm{cr}}-1,1\right)$ & & & $\mathrm{X}$ & $\mathrm{X}$ & \\
\hline$\left(\mathrm{m}_{\mathrm{cr}}-1,{ }^{*}\right)$ & & & $\mathrm{X}$ & $\mathrm{X}$ & \\
\hline$\left(\mathrm{m}_{\mathrm{cr}}+1,1\right)$ & & & $\mathrm{X}$ & $\mathrm{X}$ & \\
\hline$\left(\mathrm{m}_{\mathrm{cr}}+1,{ }^{*}\right)$ & & & $\mathrm{X}$ & $\mathrm{X}$ & \\
\hline$\left(\mathrm{m}_{\mathrm{cr}}+2,1\right)$ & & & & $\mathrm{X}$ & $\mathrm{X}$ \\
\hline$\left(\mathrm{m}_{\mathrm{cr}}+2,{ }^{*}\right)$ & & & & $\mathrm{X}$ & $\mathrm{X}$ \\
\hline
\end{tabular}

Mode Set I is assumed to be the most approximate representation of the postbuckling deformations, and its ability to predict elastic limit loads is investigated by performing para- 
metric studies on a simple panel configuration. An eight blade-stiffened panel is modelled using a stiffener-unit representation with symmetry conditions applied at the longitudinal edges of the unit cell (see Figure 7):

$$
\begin{aligned}
& f_{x}=0, \\
& \frac{\partial v}{\partial x}=0, \\
& f_{z}=0, \\
& \frac{\partial w}{\partial y}=0 .
\end{aligned}
$$

A unit axial resultant load $\hat{N}_{x G}$ is applied at the panel ends. The material properties appear in Table 3. Both skin and stiffener have a $\left[ \pm 45_{n_{1}} / 90_{n_{2}} / 0_{n_{3}}\right]_{S}$ laminate stacking sequence. The possible parameters include blade height (b) and the thicknesses of the $\pm 45^{\circ}$ plies, $90^{\circ}$ plies, and $0^{\circ}$ plies in the skin and blade laminates $\left(t_{1}, t_{2}, t_{3}, t_{4}, t_{5}, t_{6}\right.$ ) (see Figure 7b). This same panel configuration is used later in Chapter 4 for the design studies.

Table 3: Typical Graphite-Epoxy Lamina Material Values

\begin{tabular}{|cc||cc|}
\hline \multicolumn{2}{|c||}{ Material Properties } & \multicolumn{2}{c|}{ Strain Allowables } \\
\hline Young's Modulus, $\mathrm{E}_{1}$ & $20 \mathrm{Msi}$ & $\varepsilon_{1 \text { crit }}$ (tension) & 0.0105 \\
\hline Young's Modulus, $\mathrm{E}_{2}$ & $1.30 \mathrm{Msi}$ & $\varepsilon_{1 \text { crit }}$ (compression) & 0.009 \\
\hline Shear Modulus, $\mathrm{G}_{12}$ & $1.03 \mathrm{Msi}$ & $\varepsilon_{2 \text { crit }}$ (tension) & 0.00577 \\
\hline Poisson's ratio, $v_{12}$ & 0.3 & $\varepsilon_{2 \text { crit }}$ (compression) & 0.0231 \\
\hline \multicolumn{2}{|c||}{ Density, $\rho$} & $\gamma_{12 \text { crit }}$ & 0.0131 \\
\hline \multicolumn{3}{|c|}{ Ply thickness, $\mathrm{t}_{\mathrm{ply}}=0.006 \mathrm{in}$} \\
\hline
\end{tabular}

In Mode Set I, the first global mode had been assumed to remain symmetric, that is, it buckles into one half-wavelength along the $X$-direction and the $Y$-direction. A parametric 
(a)

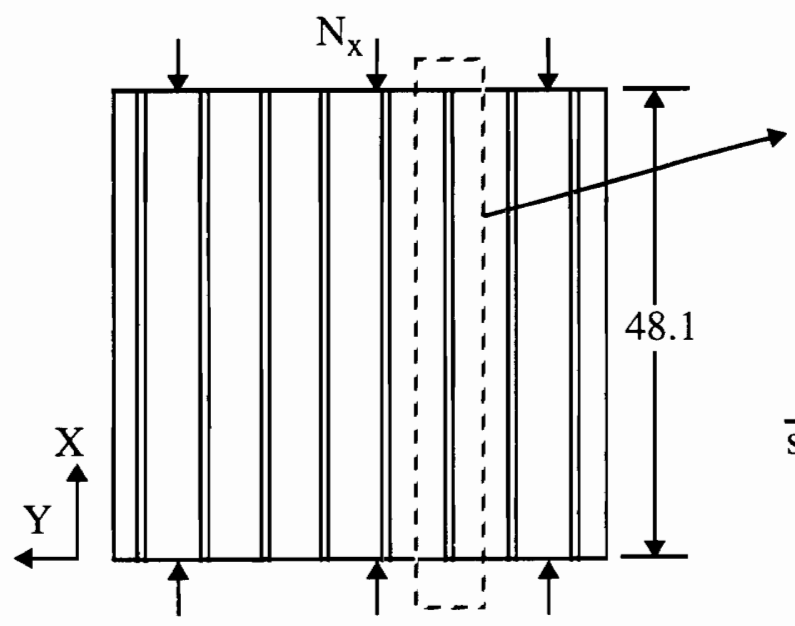

(b) stiffener-unit representation
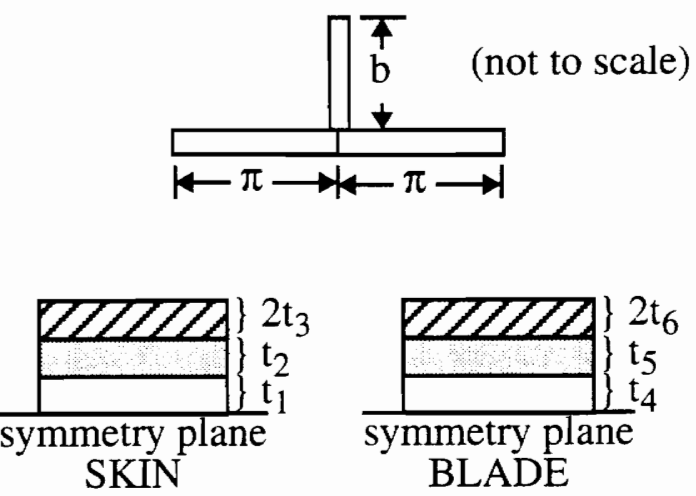

BLADE

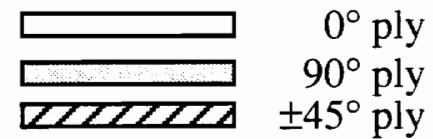

FIGURE 7. Blade-stiffened panel geometry and possible parameters. (a) Eight blade-stiffened composite panel. (b) Stiffener-unit representation with sample parameters. All dimensions are in inches. 
study of the stiffener-unit configuration (see Figure 7) showed that the first global buckling mode can sometimes become antisymmetric. Therefore, in Mode Set II in addition to the primary mode, a secondary mode with a half-wavelength of $\lambda=L$ is included to account for the primary mode becoming antisymmetric. For example, when the blade height, $b$, is varied from 1 in. to 3 in. with the skin and blade stacking sequences held to $\left[ \pm 45_{1} / 0_{1} / 90_{1}\right]$, the primary global mode is observed to become antisymmetric for blade heights greater than $2.5 \mathrm{in}$. The predicted elastic limit load as a function of the blade height for Mode Set I and Mode Set II (see Table 2) are shown in Figure 8. An elastic limit load is not detected (an elastic limit load of $2500 \mathrm{lb} / \mathrm{in}$ is a preset cut-off value in the analysis) with Mode Set I when the primary global mode becomes antisymmetric. The crosssectional buckling mode shapes for the antisymmetric global mode, the symmetric global mode and the critical local mode with a half-wavelength number of 7 are plotted at the panel midlength for a blade height of 2.6 in. in Figure 9. The antisymmetric global mode is a twisting type mode and is not affected by the local bending in the same manner as the symmetric global mode. The amount of participation each buckling mode has in representing the displacements in the nonlinear regime of response is governed by the value of each buckling mode's corresponding modal amplitude. The modal amplitudes for Mode Set I (indicated by open symbols and dashed lines) and Mode Set II (indicated by filled symbols and solid lines) as a function of end shortening for the nonlinear analysis for a blade height of 2.6 in. is shown in Figure 10. For Mode Set I, there is no contribution to the postbuckling shape from the modes other than the local critical mode $(7,1)$ which leads to an incorrect representation. For Mode Set II, on the other hand, all the modes contribute to the postbuckling displacements except for the antisymmetric global mode. Therefore, if the global mode becomes antisymmetric, a second global mode which is symmetric is added to the previous mode set. 


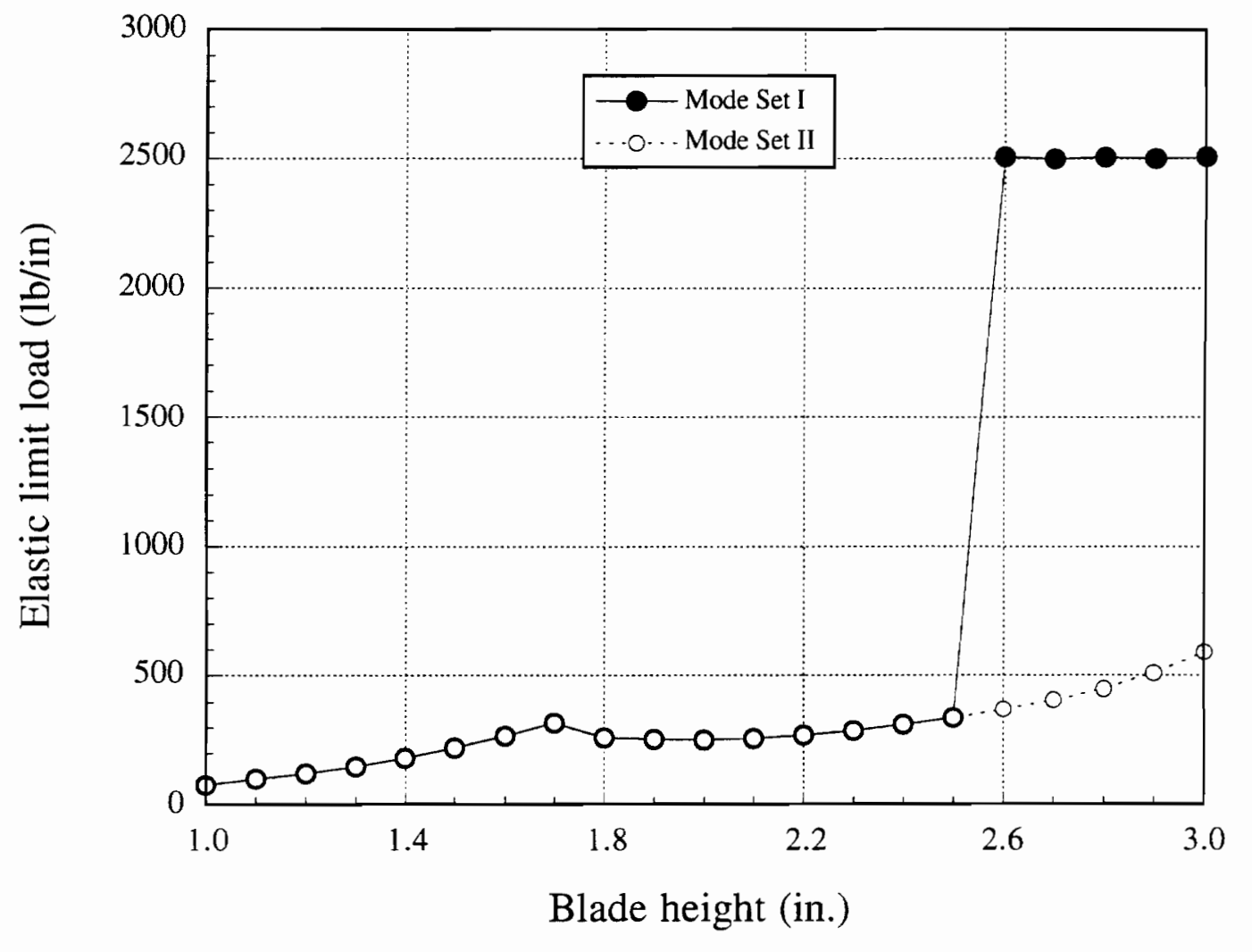

FIGURE 8. Parametric study of elastic limit load with and without symmetric global mode in mode set. 


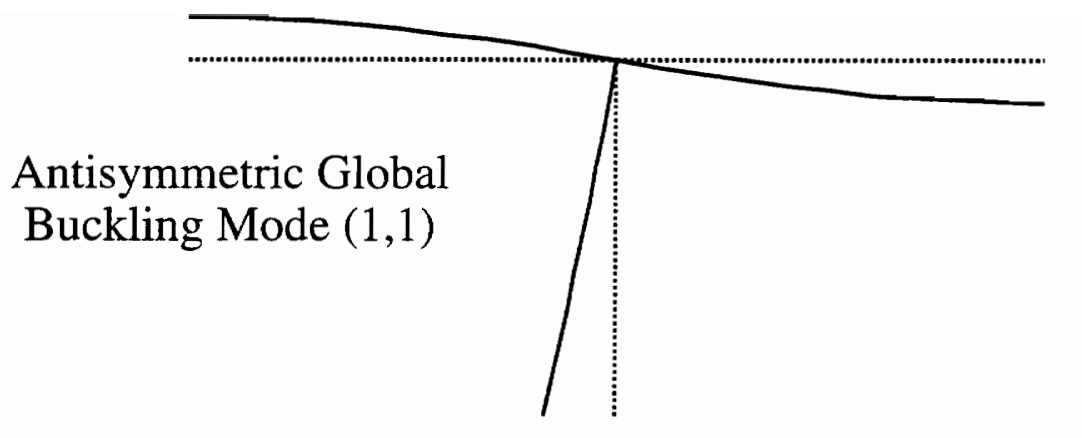

Symmetric Global

Buckling Mode (1,2)
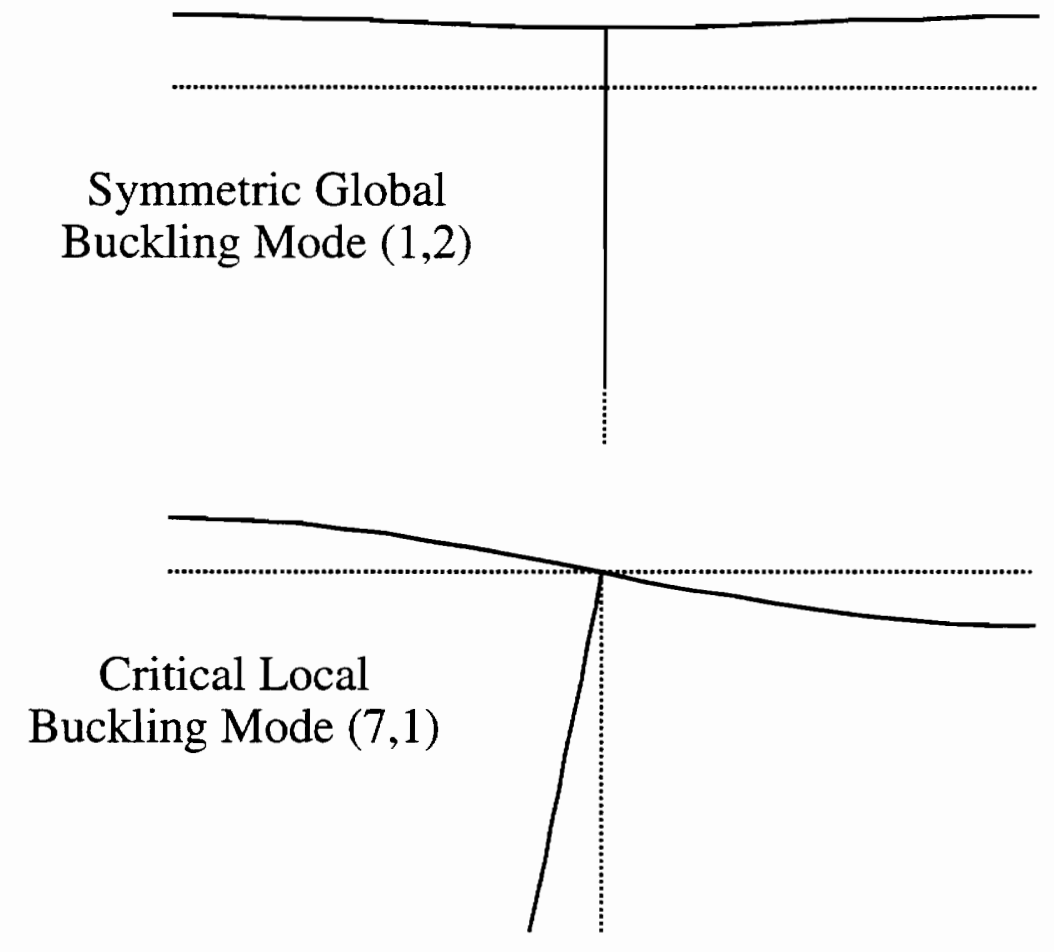

FIGURE 9. Cross-sectional buckling mode shapes at panel midlength for a blade height of 2.6 in. in Figure 8. 


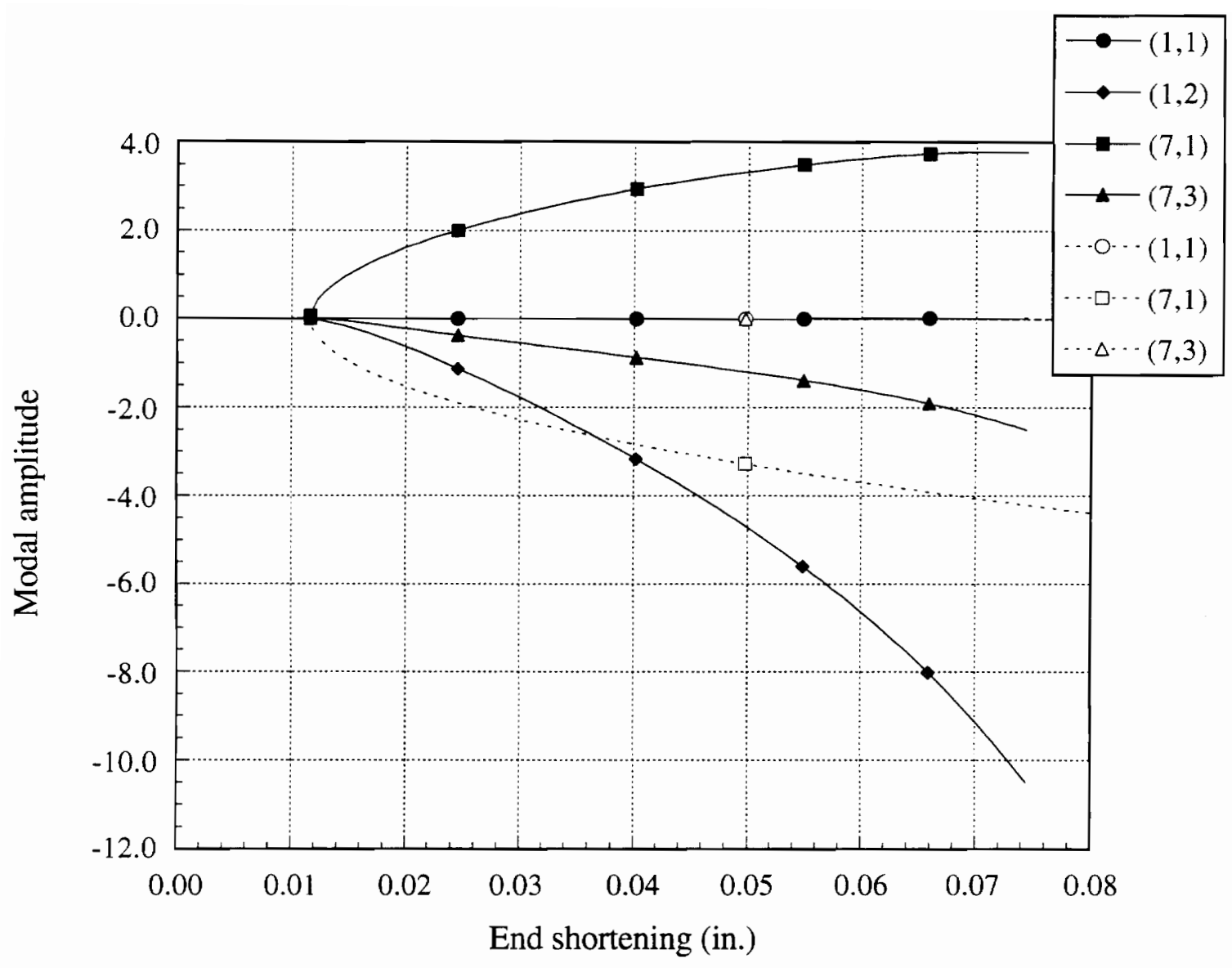

FIGURE 10. Comparison of modal amplitudes for Mode Set I (open symbols) and Mode Set II (filled symbols) at a blade height of 2.6 in. 
Changes in the buckling eigenmodes associated with the critical local mode as the design configuration changed prompted the investigation into other mode sets that included additional local modes. Three additional mode sets, Mode Set III, Mode Set IV, and Mode Set $\mathrm{V}$ in Table 2, were used in a parametric study to observe the effect of including buckling modes that are close to the critical local buckling mode in half-wavelength number. In addition to the modes in Mode Set II, Mode Set III includes the buckling modes with halfwavelength numbers $m_{c r} \pm 1$ and their secondary buckling modes, while Mode Set IV includes the buckling modes with half-wavelength numbers $\mathrm{m}_{\mathrm{cr}} \pm 1$ and $\mathrm{m}_{\mathrm{cr}} \pm 2$ and their secondary buckling modes. Mode Set V includes the buckling modes with half-wavelength numbers $\mathrm{m}_{\mathrm{cr}} \pm 2$ and their secondary buckling modes. The parametric study was performed over a range of $0^{\circ}$ blade ply thicknesses, $t_{4}$. The skin had a $\left[ \pm 45_{2} / 90_{1} / 0_{1}\right]_{S}$ laminate stacking sequence and the blade had a $\left[ \pm 45_{1} / 90_{1} / 0_{x}\right]_{S}$ laminate stacking sequence where $x$ is varied parametrically. The blade height was fixed at $1.5 \mathrm{in}$. The predicted elastic limit load as a function of the $0^{\circ}$ blade ply thickness is plotted in Figure 11 for Mode Set II, Mode Set III, Mode Set IV, and Mode Set V. The critical local half-wavelength number changed from 8 to 9,10 , and 11 as the configuration changed at a $0^{\circ}$ blade ply thickness of 0.048 in., 0.06 in., and 0.078 in., respectively, as listed in bold letters next to each point. Comparing mode set results in Figure 11, the largest difference between Mode Set II and Mode Set IV occurs at a ply thickness of 0.036 in. (see Figure 11b) where the elastic limit load for Mode Set II is 3.1\% greater than that for Mode Set IV. The largest difference between Mode Set III and Mode Set IV also occurs at a ply thickness of 0.036 in. where the elastic limit load for Mode Set III is $1.2 \%$ greater than that for Mode Set IV. No difference, however, occurs between Mode Set IV and Mode Set V. Comparing the difference in computational times, the CPUtime on the CONVEX C240 machine at NASA Langley is 13 seconds/analysis for Mode Set II, 91 seconds/analysis for Mode Set III and Mode Set V, and 381 seconds/analysis for Mode Set IV. 
(a)

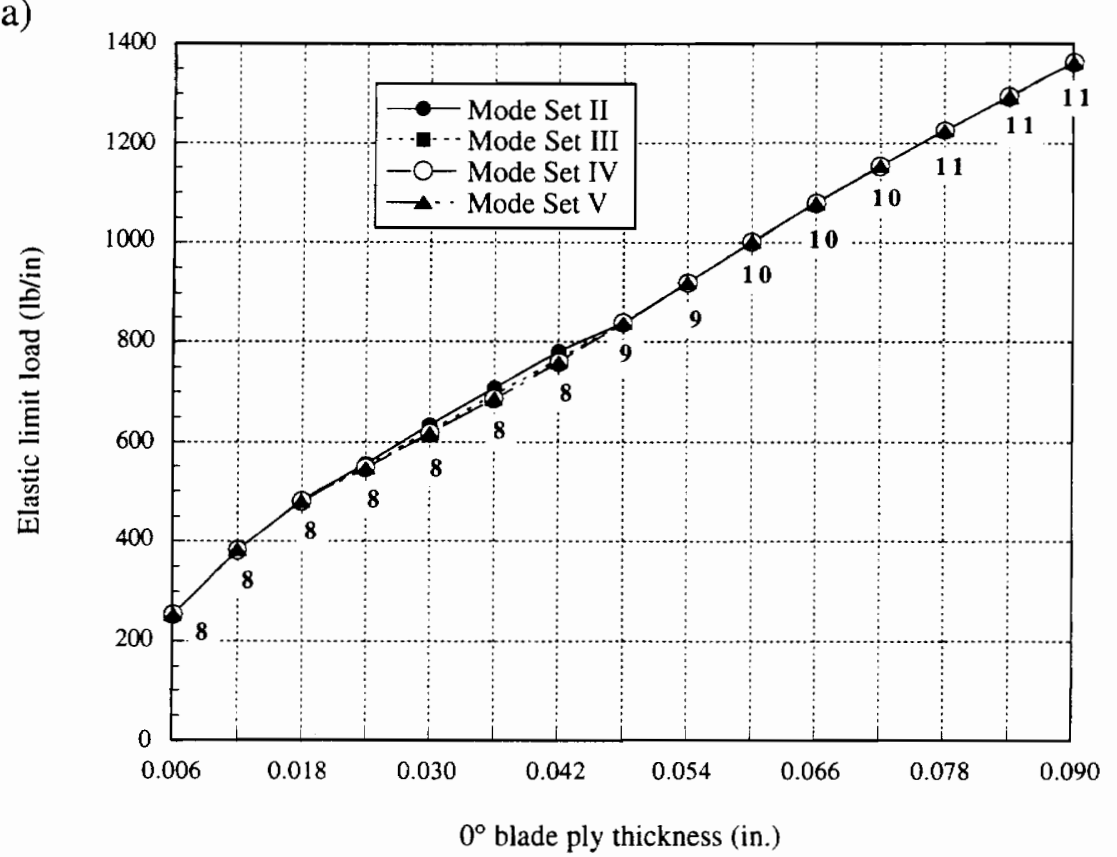

(b)

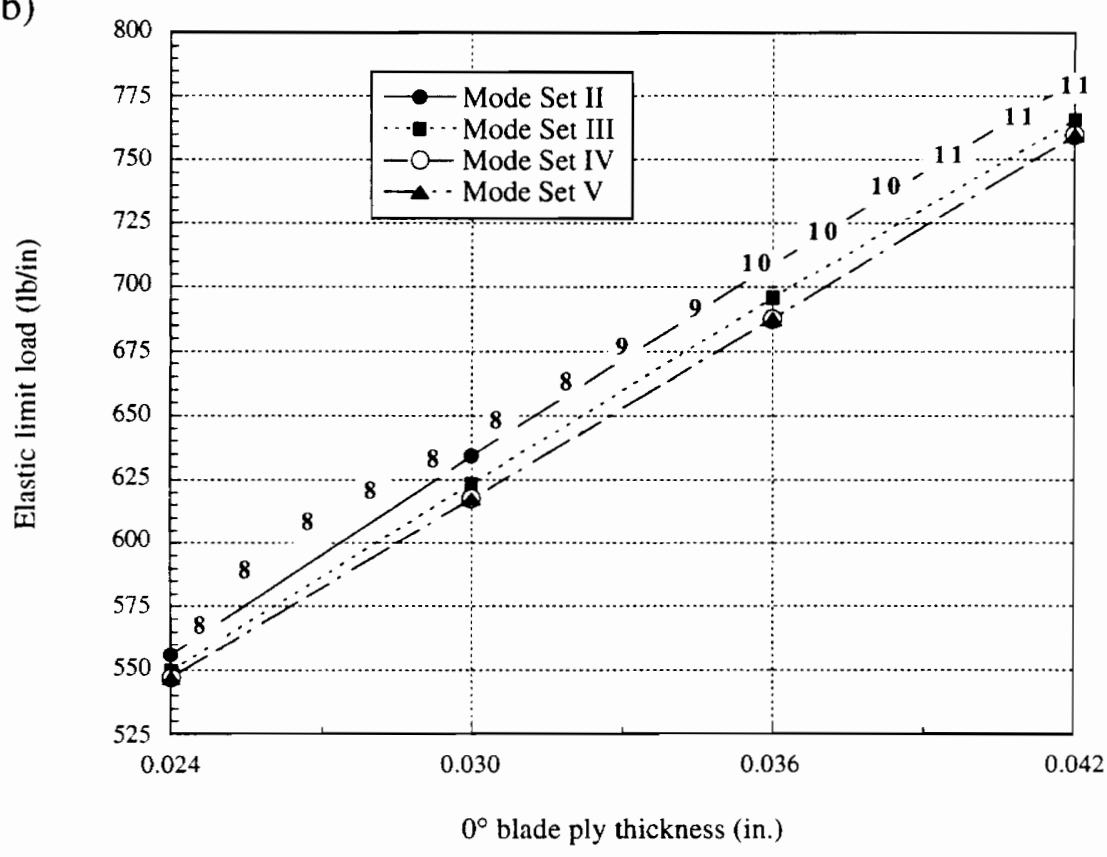

FIGURE 11. (a) Comparison of predicted elastic limit load using Mode Set II, Mode Set III, Mode Set IV, and Mode Set $\mathrm{V}$ for a range of $0^{\circ}$ blade ply thicknesses. (b) Close-up of region with largest discrepancies between mode sets (critical local half-wavelength numbers are shown by bold numbers next to each point). 
With these results, it seems logical to select Mode Set II since it predicted reasonable elastic limit load behavior with the shortest amount of computational time, however the prediction of strains also proves to be important. Figure 12 shows the maximum strain in the panel in the direction of the fibers normalized by the corresponding critical strain as a function of the $0^{\circ}$ blade ply thickness. Even though all the above mode sets gave reasonable predictions for the elastic limit load behavior, the same is not true for predicting maximum strains. Between the $0^{\circ}$ blade ply thickness values of 0.012 in. and 0.054 in., Mode Set II and Mode Set III overpredict the maximum strain in the fiber direction. At a $0^{\circ}$ blade ply thickness of 0.036 in., Mode Set III predicts a maximum strain $103 \%$ greater than Mode Set IV. At a $0^{\circ}$ blade ply thickness of 0.042 in., Mode Set II predicts a maximum strain 67\% greater than Mode Set IV. Mode Set V predicts the same values as Mode Set IV therefore, Mode Set $\mathrm{V}$, which includes additional modes with the same symmetry as the critical local buckling mode along the length of the panel, was implemented as the mode set selection strategy in NLPANOPT for the design studies in Chapter 4.

In the previous parametric study, little difference was seen in the prediction of the elastic limit load behavior for the different mode sets. This agreement between mode sets is not the case for all configurations. For example, another parametric study of the panel shown in Figure 7 was performed for a range of $0^{\circ}$ blade ply thicknesses. The skin had a $\left[ \pm 45_{1} / 90_{1} / 0_{1}\right]_{S}$ laminate stacking sequence and the blade had a $\left[ \pm 45_{1} / 90_{1} / 0_{x}\right]_{S}$ laminate stacking sequence where $x$ was varied parametrically. The blade height was fixed at $2.37519 \mathrm{in}$. The results of the predicted elastic limit load as a function of the $0^{\circ}$ blade ply thickness are plotted in Figure 13. The difference in predicted elastic limit loads between Mode Set II and Mode Set V range from 1.7\% at a ply thickness of 0.12 in. to $40 \%$ at a ply thickness of 0.093 in. The difference in predicted elastic limit loads between Mode Set III and Mode Set V range from $1.4 \%$ at a ply thickness of 0.117 in. to $32 \%$ at a 


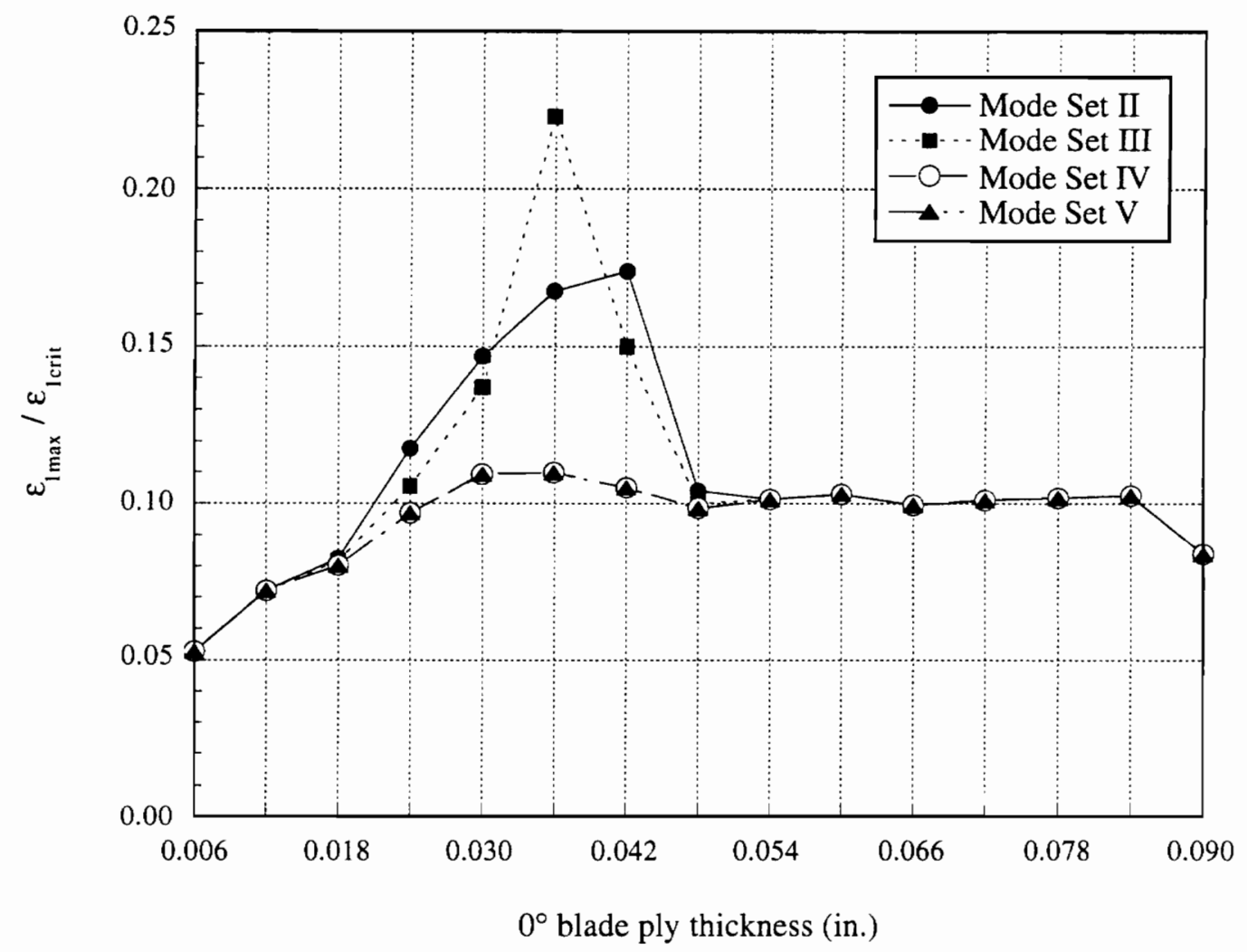

FIGURE 12. Comparison of predicted maximum axial strain in panel using Mode Set II, Mode Set III, Mode Set IV, and Mode Set V for a range of $0^{\circ}$ blade ply thicknesses. 


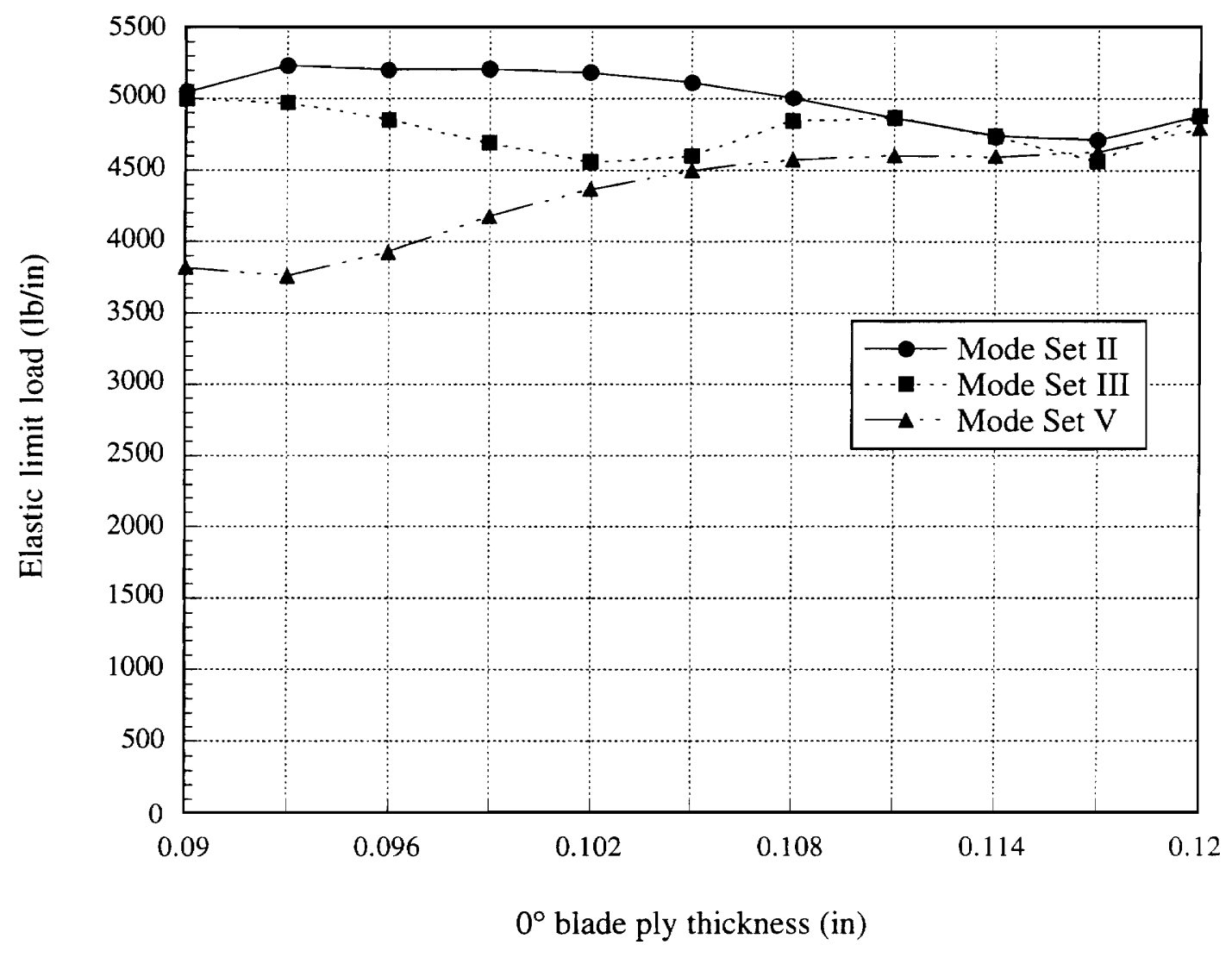

FIGURE 13. Comparison of predicted elastic limit load using Mode Set II, Mode Set III, and Mode Set $\mathrm{V}$ for a range of $0^{\circ}$ blade ply thicknesses. 
ply thickness of 0.093 in. The differences in the elastic limit load values and slopes cannot be neglected and Mode Set $\mathrm{V}$ is required as it forms the lower bound for the mode sets under consideration.

\subsection{Initial Imperfections}

NLPAN accounts for initial imperfections in the shapes of included buckling modes. As stated in Section 2.1.3, for an unloaded panel $(\lambda=0)$, the displacements degenerate to the imperfection shape of the panel:

$$
\left\{u^{o}\right\}=q_{i}^{o}\left\{u_{i}\right\}+q_{i}^{o} q_{j}^{o}\left\{u_{i j}\right\}, \quad i, j=1,2,3, \ldots
$$

where $q_{i}^{o}$ is the modal imperfection amplitude specified by the designer for the $i^{\text {th }}$ buckling mode.

Two areas, regarding initial imperfections, need to be discussed. First, since the mode shapes are normalized within NLPAN by a reference thickness, the imperfection amplitudes are also normalized values and are represented as multiples of this reference thickness. In a design study, however, this reference thickness is changing with the design. Therefore, a constant value or multiple of a constant dimension such as the panel length is preferred for specifying the initial imperfection amplitudes. For this reason, the global modal imperfection amplitude is entered as a percentage of the panel length and the local modal imperfection amplitudes are entered as constant values. Second, the shape of the local imperfection is changing throughout the design space with the change in the critical local buckling mode and its half-wavelength number. This limitation is not considered a major problem since it is assumed that the imperfections in the major modes are most important, so a worst case design is always found.

The fact that NLPAN includes initial imperfections is beneficial not only for imperfection 
sensitivity in modal interaction behavior, but also for simplifying the branching between equilibrium paths. This aspect of the nonlinear analysis is discussed further by Stoll [5]. It should be stated that too small an imperfection causes branching problems, but too large an imperfection leads to inaccuracies. A study by Stoll [1] found that NLPAN fails to predict an asymptotic elastic limit load for an increasing global imperfection amplitude. For this study, a zero imperfection amplitude is taken to be $0.0001 \%$ of the panel length for the global mode and 0.0001 times the initial reference thickness for the local mode and a maximum imperfection amplitude is taken to be $0.1 \%$ of the panel length for the global mode and 0.1 times the initial reference thickness for the local mode.

Both positive and negative imperfection amplitudes in the shape of the global mode are checked for each analysis. Thus, the design is not driving the panel to either direction when it is not known which way the panel will be driven physically. The effect of accounting for both positive and negative global imperfection shapes is shown in Figure 14. An elastic limit load is predicted over a range of $\pm 45^{\circ}$ ply thicknesses, $\mathrm{t}_{6}$, in the blade. The skin has a $\left[ \pm 45_{1} / 90_{1} / 0_{1}\right]_{S}$ laminate stacking sequence and the blade has a $\left[ \pm 45_{x} / 90_{1.5} / 0_{1}\right]_{S}$ laminate stacking sequence where $x$ is varied parametrically. The blade height is $1.9 \mathrm{in}$. The predicted elastic limit load is plotted as a function of the $0^{\circ}$ blade ply thickness. The results for a positive global imperfection amplitude of $0.0481 \mathrm{in}$. ( $0.1 \%$ of the panel length) are shown by the filled circle solid line. The results for the same magnitude but opposite direction of the global imperfection amplitude are shown by the filled square dashed line. For this configuration, the elastic limit load predicted for the negative imperfection is used until a ply thickness of $0.01 \mathrm{in}$., then the positive imperfection result is used. 


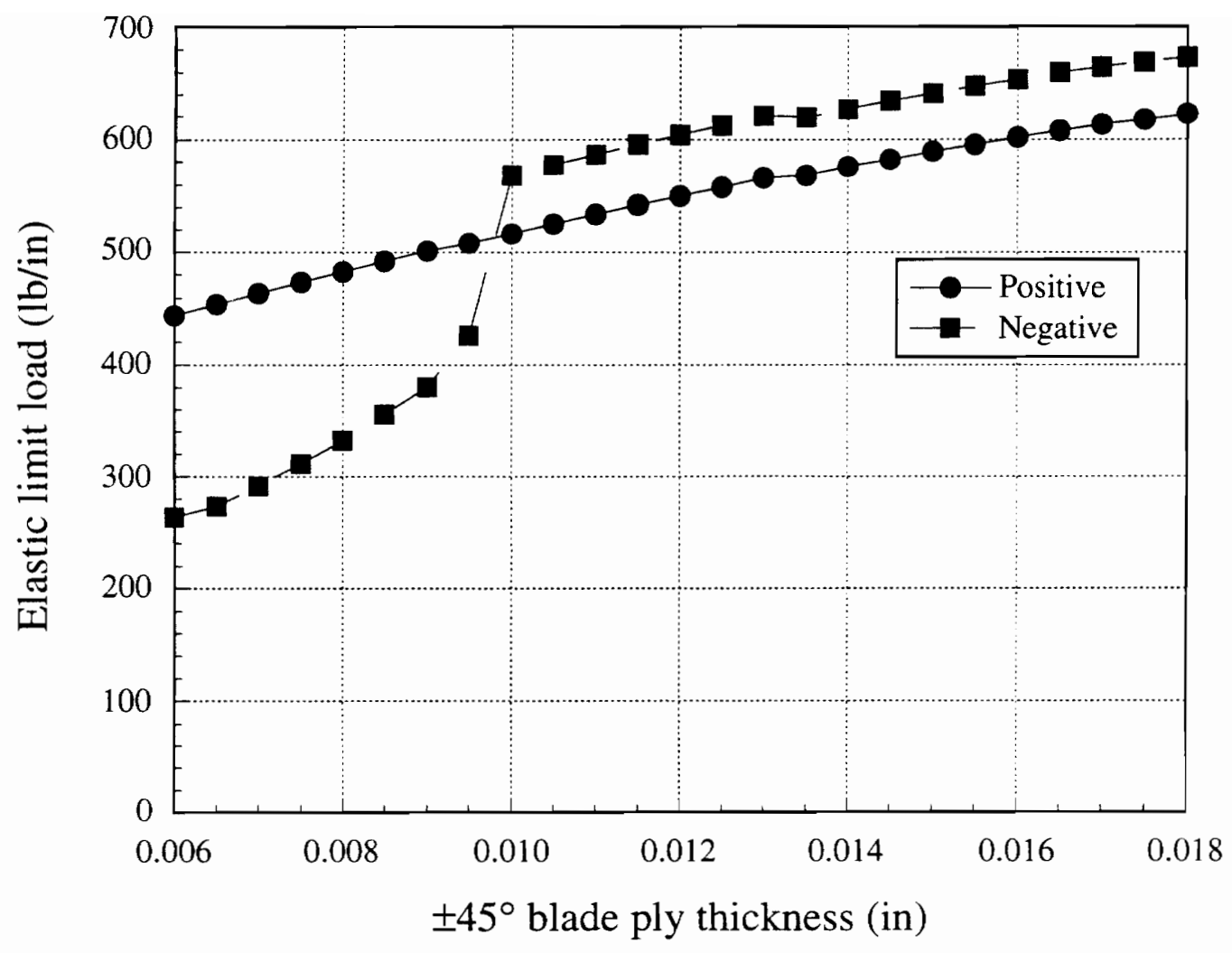

FIGURE 14. Parametric study of elastic limit load using Mode Set I with a positive and negative global imperfection shape. 


\subsection{Design Formulation}

With the method of analysis defined, the development of the design procedure is described in this chapter. First, the program organization is described followed by a brief discussion of the optimization program used. Finally, a statement of the minimization problem is presented with a discussion of code refinements necessary for automated design.

\subsection{Program Organization}

The design procedure, NLPANOPT, is an assembly of an integrated analysis and an optimization algorithm. A schematic of the program organization is shown in Figure 15. The integrated analysis contains two codes: NLPAN and PASCO. NLPAN is the nonlinear analysis code described in the previous chapter (see [4]). This nonlinear analysis method uses buckling eigenfunction information from PASCO, determined within its analysis section, VIPASA. The input file for PASCO is generated with a Macintosh-based, interactive graphic preprocessor, MacPASCO [45]. Information about the panel weight and response from the integrated analysis is relayed through the controlling program, NLPANOPT, to the optimization program, ADS. This information is then used to move through the design space until an optimum panel geometry is found. Many cycles are performed relaying information from the integrated analysis to the optimization algorithm and vice-versa. 


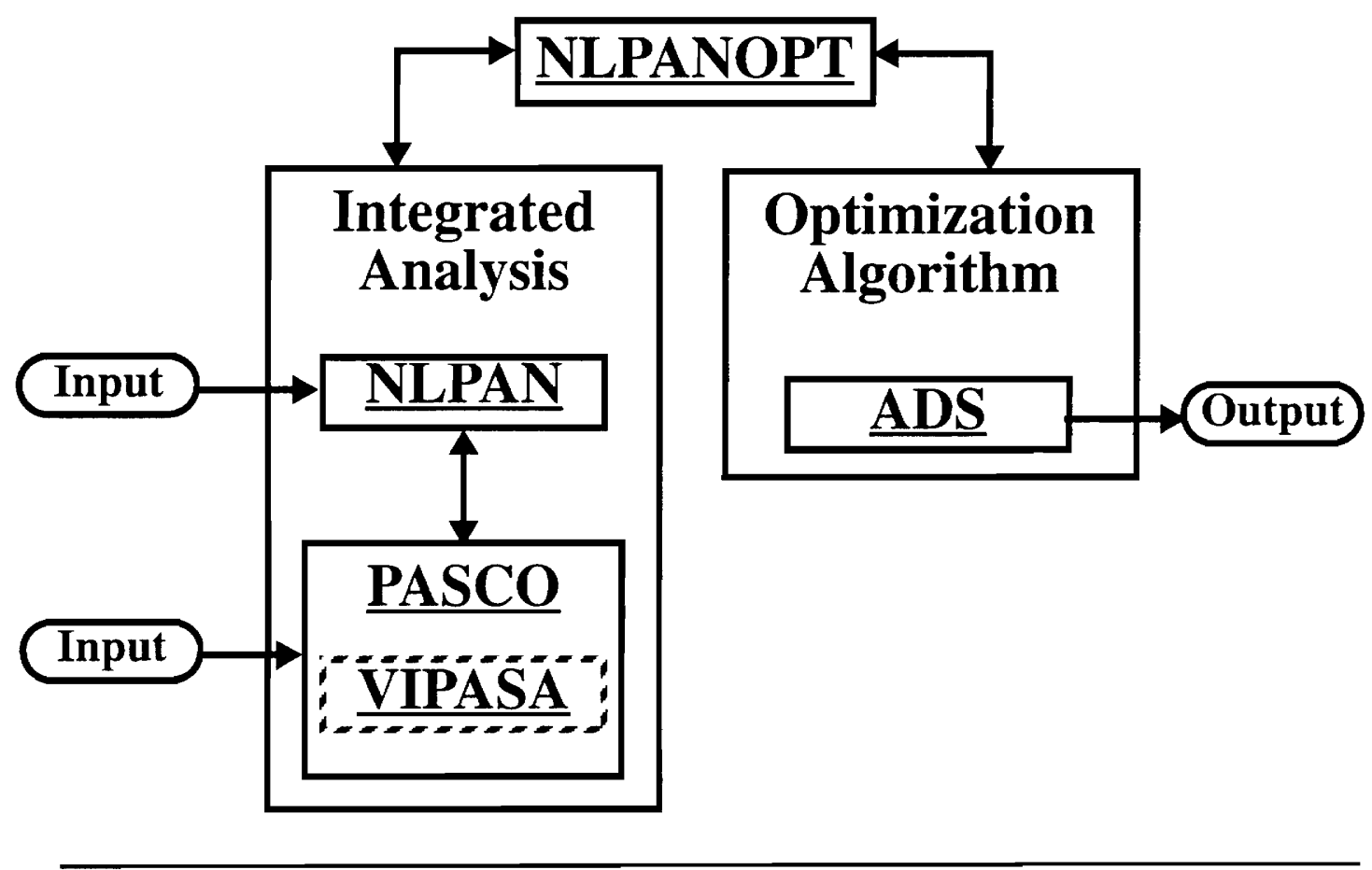

FIGURE 15. NLPANOPT program organization. 


\subsection{Optimization Program - ADS}

ADS is a general purpose numerical optimization program for engineering design developed by G.N. Vanderplaats [9]. The optimization problem is posed in the following form:

$$
\begin{aligned}
& \text { Minimize } \quad F(\mathbf{X}), \\
& \text { Subject to } \quad g_{j}(\mathbf{X}) \leq 0 \quad j=1, \mathrm{~m}, \\
& \quad \mathrm{X}_{i}^{\text {min }} \leq \mathrm{X}_{i} \leq \mathrm{X}_{i}^{\text {max }} \quad i=1, \mathrm{n},
\end{aligned}
$$

where $\mathbf{X}$ is the vector of design variables, $F$ is the objective function to be minimized, $g_{j} \leq 0$ are the constraints on the design space, and $X_{i}^{\text {min }}$ and $X_{i}^{m a x}$ are the bound constraints on a particular design variable.

ADS is segmented into three levels: strategy, optimizer, and one-dimensional search, with several options available at each level. For this study, the constraints are treated directly without the need for a strategy, the optimizer is the Method of Feasible Directions, and the one-dimensional search is polynomial interpolation with bounds found first. The design procedure begins at a specified initial set of design variables, $\boldsymbol{X}^{\circ}$; the design is updated iteratively in the following form:

$$
\mathbf{X}^{q+1}=\mathbf{X}^{q}+\alpha^{*} \mathbf{S}^{q}
$$

where $q$ is the iteration number, $\mathbf{S}^{q}$ is the search direction vector, and $\alpha^{*}$ is a scalar defining the distance to move along $\mathbf{S}^{q}$. The search direction is found first using the optimizer, then the scalar distance is found using the one-dimensional search.

\subsection{Statement of Minimization Problem}

In this study, the design problem is to minimize the (weight / area) of a linked-plate panel such that it will sustain a specified design load, $N_{D}$, without failure due to exceeding the 
elastic limit load, $N_{L}$, or local material failure. For a general configuration, the design variables include the plate-strip widths, $b_{i}$, and the ply thicknesses, $\left(t_{j}\right)_{i}$. For a panel of constant length, $L$, composed of $P$ plates, each having an independent number of plies, $N_{i}$, the minimum weight design problem is stated in equation form (similar to Eqn. 29) as

$$
\begin{aligned}
& \text { Minimize } \quad F(\mathbf{X})=\frac{W}{A L}=\frac{\sum_{i=1}^{P} b_{i}\left(\sum_{j=1}^{N_{i}} \rho_{j} t_{j}\right)}{B L^{2}}, \\
& \text { Subject to } \quad g_{1}(\mathbf{X})=1.0-\frac{N_{L}}{N_{D}} \leq 0.0, \\
& g_{2}(\mathbf{X})=1.0-\frac{\varepsilon_{1 \text { crit }}}{\varepsilon_{1 \max }} \leq 0.0, \\
& g_{3}(\mathbf{X})=1.0-\frac{\varepsilon_{2 c r i t}}{\varepsilon_{2 \max }} \leq 0.0, \\
& g_{4}(\mathbf{X})=1.0-\frac{\gamma_{12 c r i t}}{\gamma_{12 \max }} \leq 0.0, \\
&\left\{\mathbf{X}^{\min }\right\} \leq\{\mathbf{X}\} \leq\left\{\mathbf{X}^{\max }\right\},
\end{aligned}
$$

where the design variables are $\{\mathbf{X}\}=\left(b, t_{j}\right)_{i},\left(\rho_{j}\right)_{i}$ is the density of each ply, $\varepsilon_{1 \text { max }}$ is the maximum axial strain found in the panel, $\varepsilon_{1 \text { crit }}$ is the allowable axial strain, $\varepsilon_{2 \max }$ is the maximum transverse strain found in the panel, $\varepsilon_{2 c r i t}$ is the allowable transverse strain, $\gamma_{12 \max }$ is the maximum shear strain found in the panel, and $\gamma_{12 \text { crit }}$ is the allowable shear strain.

The nonlinear analysis detects elastic limit loads, thus this constraint information is readily available. For material failure constraint information, a failure criterion during the post-processing of NLPAN is necessary. Experimental studies show that failure of stiff- 
ened panels generally occurs through stiffener debonding due to interlaminar stresses. NLPAN, with the classical laminated plate theory at its foundation, neglects interlaminar strains, thus this failure mode cannot be predicted directly. For this study, material failure is predicted by a maximum strain failure criterion. Failure is assumed to occur if one of the following conditions is satisfied in any ply within a laminate:

$$
\begin{gathered}
\varepsilon_{1}>\varepsilon_{1 c r i t}^{t}, \quad \varepsilon_{1}<\varepsilon_{1 c r i t}^{c}, \\
\varepsilon_{2}>\varepsilon_{2 c r i t}^{t}, \quad \varepsilon_{2}<\varepsilon_{2 c r i t}^{c}, \\
\left|\gamma_{12}\right|>\gamma_{12 \text { crit }},
\end{gathered}
$$

where $\varepsilon_{1 \text { crit }}^{t}$ and $\varepsilon_{1 \text { crit }}^{c}$ are the allowable tensile and compressive strain values in the fiber direction of a laminate, respectively, while $\varepsilon_{2 c r i t}^{t}$ and $\varepsilon_{2 c r i t}^{c}$ are the allowable tensile and compressive strain values in the direction perpendicular to the fiber direction, respectively, and $\gamma_{12 \text { crit }}$ is the allowable shearing strain.

\subsection{NLPAN Refinements}

In order to develop an automated design tool, refinements or additions to the nonlinear analysis program are necessary. This section starts with a discussion of material failure constraint calculations, followed by gradient information through finite difference calculations.

\subsubsection{Material Failure Constraint}

From Eqn. (31), the calculation of the material failure constraints, $g_{2}(\mathbf{X}), \mathrm{g}_{3}(\mathbf{X})$, and $\mathrm{g}_{4}(\mathbf{X})$, requires maximum strain values. During an analysis, the strain calculations are made at a set of points within the plate-strip laminates which are specified as input information to the program NLPAN. These strain values are then scanned at one load for each analysis (see following paragraph) to find the maximum strain values in the plate for each 
principal material direction. The constraint equation is then solved for each direction. For the strains in the fiber direction and perpendicular to the fiber direction, the constraint equation is evaluated for both tensile and compressive maximum values, since the allowables are different. Then the most active constraint (that which is most positive) is selected for each direction.

Three types of panel response are possible in a single nonlinear analysis within a design run (see Figure 16). The first type is when an elastic limit load occurs before the specified design load. The maximum strain in the panel is determined at this limit load. The second type is when an elastic limit load occurs after the specified design load. The maximum strain in the panel is determined at the specified design load, then the analysis continues until the limit load is found. The third type of response is when no elastic limit load is calculated in the panel before a set cut-off load value (multiple of the specified design load). Again, the maximum strain is determined at the specified design load and the elastic limit load is taken to be this cut-off value. Gradient information is not affected by this bogus elastic limit load value since only active or violated constraints are included for a specific iteration.

\section{$\underline{\text { 3.4.2 Gradient Calculations }}$}

The gradient information necessary for the Method of Feasible Directions is found within ADS using the first-order forward-difference approximation. Given a function $u(x)$ of a design variable $x$, the forward-difference approximation $\Delta u / \Delta x$ to the derivative $d u / d x$ is given as

$$
\frac{\Delta u}{\Delta x}=\frac{u(x+\Delta x)-u(x)}{\Delta x}
$$




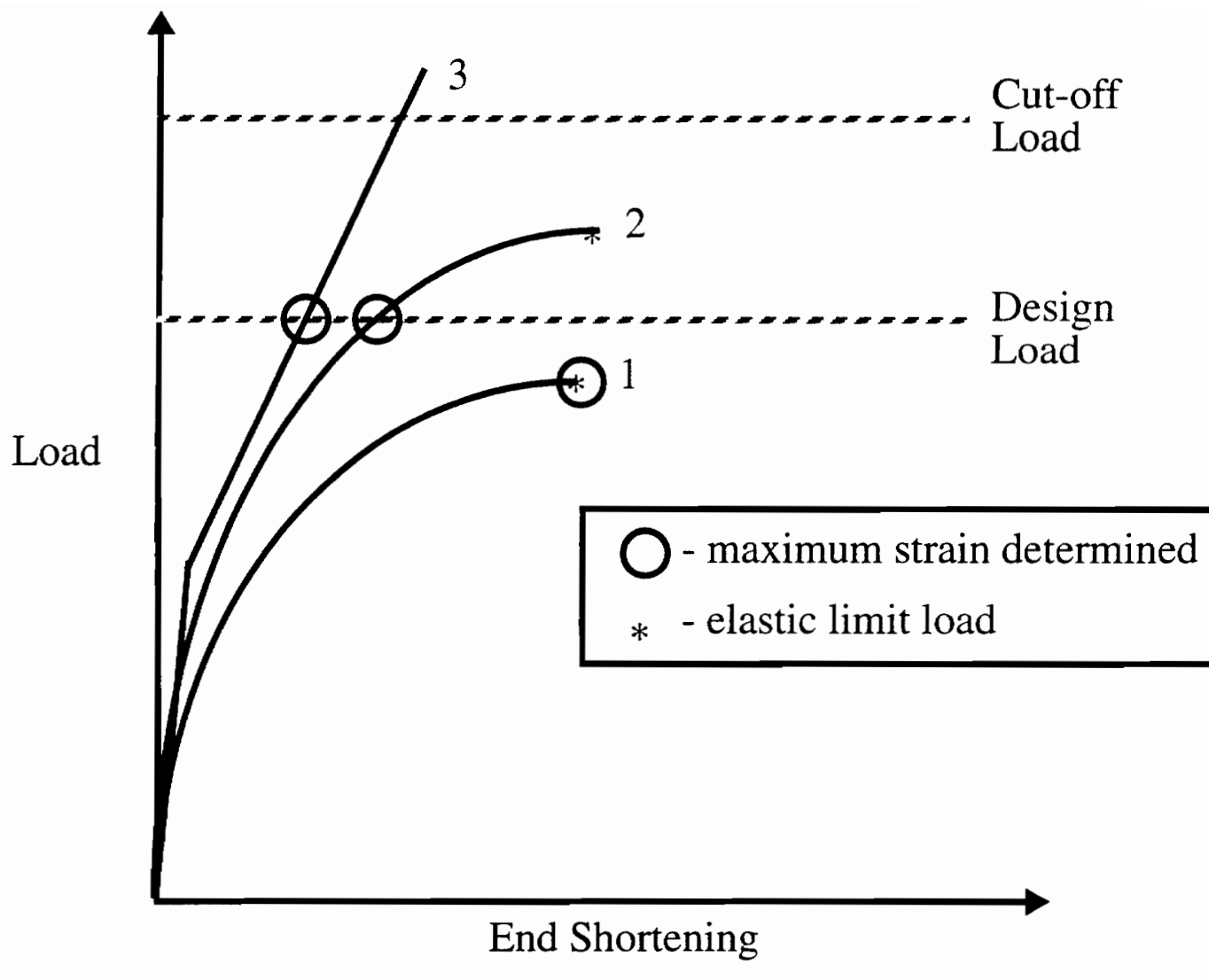

FIGURE 16. Possible panel responses for constraint value determination. 
Each design variable $X_{k}$ is perturbed separately and the nonlinear analysis rerun to obtain the gradient of the objective function and active or violated constraint functions. An important decision in the finite difference calculation is the step-size $\Delta x$ to use. Since the design variables may differ by magnitudes (i.e., a ply thickness compared to a plate width), the step-size is taken as a percentage change in the design variables as opposed to a direct change. Through extensive studies during this work, comparing first-order forward-difference calculations with second-order central-difference calculations over a wide range of step-sizes, a step-size of $0.01 \%$ of the design variable is used. 


\subsection{Design Study}

This chapter includes results from studies on two stiffened panel configurations. Comparisons of weight and imperfection sensitivity between the panels designed with buckling constraints and the panels designed to operate in postbuckling load regimes are made. The first configuration is the blade-stiffened panel modelled in Section 2.2 (see Figure 7). The majority of research time was spent using this simplified model. The panel is designed for four levels of axial compressive loads: $\mathrm{N}_{\mathrm{x}}=100 \mathrm{lb} / \mathrm{in}, 1000 \mathrm{lb} / \mathrm{in}, 5000 \mathrm{lb} / \mathrm{in}$, and 10000 $\mathrm{lb} / \mathrm{in}$. Then, for the $1000 \mathrm{lb} /$ in axial compressive load case, the effect of the skin laminate stacking sequence is investigated. The second configuration is similar to the first, but with added stiffener flanges. It is designed for an axial compressive load of $\mathrm{N}_{\mathrm{x}}=1000 \mathrm{lb} / \mathrm{in}$.

\subsection{Design of Blade-Stiffened Panel for Four Levels of Axial Compressive Loads}

An eight blade-stiffened graphite-epoxy panel subject to an axial compressive loading is modelled using a stiffener-unit representation with symmetry conditions applied at the longitudinal edges $(Y=0, B)$ of the unit cell. The conditions at the cell boundaries are 


$$
\begin{aligned}
& f_{x}=0, \\
& \frac{\partial v}{\partial x}=0, \\
& f_{z}=0, \\
& \frac{\partial w}{\partial y}=0^{\prime} .
\end{aligned}
$$

This is the panel discussed in Section 2.2 (see geometry in Figure 7 and material properties in Table 3). Designs are obtained for four levels of axial compressive loads of $\mathrm{N}_{\mathrm{x}}=$ $100 \mathrm{lb} / \mathrm{in}, 1000 \mathrm{lb} / \mathrm{in}, 5000 \mathrm{lb} / \mathrm{in}$, and $10000 \mathrm{lb} / \mathrm{in}$. For each loading, the panel is designed using: 1) PASCO with no imperfections, 2) PASCO with an initial bowing imperfection of 0.0481 in. (0.1\% of the panel length), 3) NLPANOPT with no imperfections, and 4) NLPANOPT with an initial imperfection in the shape of the global mode and an amplitude of 0.0481 in. (0.1\% of the panel length). The parameters discussed in Section 2.2 and shown in Figure 7 are the design variables: the blade height, b, the skin ply thicknesses, $\mathrm{t}_{1}$, $t_{2}$, and $t_{3}$ corresponding to the ply orientations of $0^{\circ}, 90^{\circ}$, and $\pm 45^{\circ}$, and the blade ply thicknesses, $\mathrm{t}_{4}, \mathrm{t}_{5}$, and $\mathrm{t}_{6}$ corresponding to the ply orientations of $0^{\circ}, 90^{\circ}$, and $\pm 45^{\circ}$, respectively. The optimization programs within PASCO and NLPANOPT use continuous design variables. Therefore, the minimum-weight designs contain ply thicknesses which are not multiples of the minimum ply thickness, $\mathrm{t}_{\mathrm{ply}}$. For practical designs, these continuous ply thicknesses are rounded-off to obtain the nearest minimum-weight feasible design. The following sections discuss only the results for the rounded-off designs, except for the $1000 \mathrm{lb} / \mathrm{in}$ and $10000 \mathrm{lb} / \mathrm{in}$ axial compressive load cases where continuous results are also presented. 


\subsubsection{Case 1: $100 \mathrm{lb} /$ in Design Load}

The design results for the $100 \mathrm{lb} / \mathrm{in}$ design load are listed in Table 4 . The first two columns represent the PASCO designs, with and without the initial bowing imperfection, and the last two columns represent the NLPANOPT designs, with and without the initial global imperfection. The design variables, $b$ and $t_{1}$ through $t_{6}$, along with the final stiffener-unit

mass are listed for each column. The global eigenvalue, $\lambda_{\text {global }}$, critical local eigenvalue, $\lambda_{\text {local }}$, and critical local half-wavelength number, $\mathrm{m}_{\text {local }}$, are also listed. Note that the ply thickness are listed as multiples of the minimum ply thickness, $\mathrm{t}_{\mathrm{ply}}=0.006$ in. (for example, $\mathrm{t}_{4}$ for the NLPANOPT design with an imperfection is $0.012 \mathrm{in}$.).

Comparing the results for panels with no initial imperfections, the buckling constrained design and the design obtained with the nonlinear analysis capability are approximately the same and are governed by the global buckling of the panel. That is, although the panel is allowed to operate in the postbuckling load regime, the optimal configuration for this low axial compressive load level is that which is buckling critical with the minimum thickness skin and minimum thickness blade plate-strips.

With the addition of initial imperfections, PASCO increases the blade height by $15 \%$, while NLPANOPT increases the blade height by less than $1 \%$ but adds a $0^{\circ}$ ply to the blade. The increase in the blade thickness in the NLPANOPT design causes a slight increase in the overall panel weight compared to the PASCO design.

By taking into account the postbuckling strength of the panel, as well as its imperfection sensitivity, the nonlinear designs were expected to be lighter and less imperfection sensitive compared to the buckling critical designs. With this light loading and design variable selection, however, the panel does not have the opportunity to reach its postbuckled state. 
Although it is not investigated in this study, more interesting results may occur if stiffener spacing is also included as a design variable.

Table 4: Summary of panel designs for $N_{x}=100 \mathrm{lb} / \mathrm{in}$.

\begin{tabular}{|c|c|c|c|c|}
\hline & PASCO & $\begin{array}{l}\text { PASCO w/ } \\
\text { imperfection }\end{array}$ & NLPANOPT & $\begin{array}{c}\text { NLPANOPT } \\
\text { w/ } \\
\text { imperfection }\end{array}$ \\
\hline b (in.) & 1.09473 & 1.26017 & 1.09499 & 1.10144 \\
\hline$t_{1}\left(0^{\circ}{ }_{\text {skin }}\right)$ & 1 & 1 & 1 & 1 \\
\hline $\mathrm{t}_{2}\left(90^{\circ}{ }_{\text {skin }}\right)$ & 1 & 1 & 1 & 1 \\
\hline $\mathrm{t}_{3}\left( \pm 45^{\circ}\right.$ skin $)$ & 1 & 1 & 1 & 1 \\
\hline $\mathrm{t}_{4}\left(0_{\text {blade }}^{\circ}\right)$ & 1 & 1 & 1 & 2 \\
\hline $\mathrm{t}_{5}\left(90^{\circ}\right.$ blade $)$ & 1 & 1 & 1 & 1 \\
\hline $\mathrm{t}_{6}\left( \pm 45^{\circ}\right.$ blade $)$ & 1 & 1 & 1 & 1 \\
\hline Unit Weight (lbs.) & 0.97094 & 0.99272 & 0.97098 & 1.00800 \\
\hline$\lambda_{\text {global }}$ & 1.0 & 1.5 & 1.0 & 1.6 \\
\hline$\lambda_{\text {local }}$ & 1.21 & 1.0 & 1.2 & 1.4 \\
\hline$\overline{\mathrm{m}_{\text {local }}}$ & 8 & 9 & 8 & 9 \\
\hline
\end{tabular}

\subsubsection{Case 2: $1000 \mathrm{lb} / \mathrm{in}$ Design Load}

The design results for the $1000 \mathrm{lb} / \mathrm{in}$ axial compressive load are listed in Table 5 with the continuous ply thicknesses before rounding-off and the associated buckling eigenvalues and weight of the panel shown in parentheses. Comparing the rounded-off results for the panels designed with no initial imperfections, the NLPANOPT design weighs approximately $28 \%$ less than the PASCO design. This weight savings stems from the reduction of $\pm 45^{\circ}$ plies in the skin and $0^{\circ}$ plies in the blade even though the blade height is increased by $26 \%$. The continuous NLPANOPT design is limit load critical with the panel in a locally postbuckled state, buckling into 10 half-wavelengths at $27 \%$ of the design load $\left(\lambda_{\text {local }}=\right.$ 
0.269). The continuous PASCO design, on the other hand, is driven to simultaneous global and local buckling at the design load.

Table 5: Summary of panel designs for $\mathbf{N}_{x}=1000 \mathrm{lb} / \mathrm{in}$.

\begin{tabular}{|c|c|c|c|c|}
\hline & PASCO & $\begin{array}{c}\text { PASCO } w / \\
\text { imperfection }\end{array}$ & NLPANOPT & $\begin{array}{c}\text { NLPANOPT } \\
\text { w/ } \\
\text { imperfection }\end{array}$ \\
\hline \hline $\mathrm{b}$ (in.) & 1.42189 & 1.82324 & 1.78738 & 1.79196 \\
\hline $\mathrm{t}_{1}\left(0^{\circ}{ }_{\text {skin }}\right)$ & $1(1)$ & $1(1)$ & $1(1)$ & $1(1)$ \\
\hline $\mathrm{t}_{2}\left(90^{\circ}{ }_{\text {skin }}\right)$ & $1(1)$ & $1(1)$ & $1(1)$ & $1(1)$ \\
\hline $\mathrm{t}_{3}\left( \pm 45^{\circ}{ }_{\text {skin }}\right)$ & $2(2.03)$ & $3(2.3)$ & $1(1)$ & $1(1)$ \\
\hline $\mathrm{t}_{4}\left(0^{\circ}{ }_{\text {blade }}\right)$ & $11(10.04)$ & $6(8.69)$ & $6(5.277)$ & $6(5.816)$ \\
\hline $\mathrm{t}_{5}\left(90^{\circ}{ }_{\text {blade }}\right)$ & $1(1)$ & $1(1)$ & $1(1)$ & $1(1)$ \\
\hline $\mathrm{t}_{6}\left( \pm 45^{\circ}{ }_{\text {blade }}\right)$ & $1(1)$ & $1(1)$ & $1(1)$ & $1(1)$ \\
\hline Unit Weight $_{(\text {lbs. })}$ & 1.895 & 2.1936 & 1.3562 & 1.3575 \\
\hline$\lambda_{\text {global }}$ & $1.8628)$ & $(2.0657)$ & $(1.3136)$ & $(1.3468)$ \\
\hline$\lambda_{\text {local }}$ & $1.05(1.0)$ & $1.11(1.0)$ & $0.293(0.269)$ & $0.293(0.287)$ \\
\hline $\mathrm{m}_{\text {local }}$ & $10(9,10)$ & $8(8,9)$ & $11(10)$ & $11(11)$ \\
\hline
\end{tabular}

Considering the above designs, one possessing simultaneous buckling modes and the other displaying local postbuckling behavior, the sensitivity of each design to imperfections was of interest. Both designs were subsequently analyzed for a range of imperfection amplitudes using the linear analysis VIPASA to determine the panel buckling load, and the nonlinear analysis NLPAN to determine the panel elastic limit load. For VIPASA analyses, a range of bowing imperfections from $-0.1 \%$ of the panel length to $+0.1 \%$ of the panel length were included. For NLPAN analyses, in addition to the same range of global imperfections, a range of local imperfections from zero to 0.1 times the skin thickness, denoted as $t$, and a combination of both global and local imperfections with the same amplitude 
were included. The results of this imperfection sensitivity study are shown (for the rounded-off designs in Table 5) in Figure 17 for the rounded-off PASCO design and Figure 18 for the NLPANOPT design. For these figures and all subsequent imperfection sensitivity figures, the buckling load for the VIPASA results and the elastic limit load for the NLPAN results are plotted as a function of the global imperfection amplitudes, $q_{g}^{o}$, and/or local imperfection amplitudes, $q_{l}^{o}$. The line with open circles denotes the buckling load from VIPASA and the line with solid circles denotes the elastic limit load from NLPAN for the range of global imperfections. The line with solid triangles denotes the predicted elastic limit load from NLPAN for the range of local imperfections and the line with solid squares denotes the predicted elastic limit load from NLPAN for the combination of global and local imperfections.

For the rounded-off PASCO design (Figure 17), the sensitivity of the panel to positive global imperfections results in buckling of the panel before the design load is reached. For a bowing imperfection amplitude of $+0.1 \%$ of the panel length, the panel buckles at a load of approximately $720 \mathrm{lb} / \mathrm{in}$. NLPAN also predicts the PASCO design to be incapable of carrying the design load for positive global imperfections. The local imperfections also cause the panel to buckle below the design load. With both global and local imperfections, the panel remains overly sensitive to the positive imperfection amplitudes.

For the rounded-off NLPANOPT design (Figure 18), the panel carries the required load for all imperfection amplitudes. An increase in the limit load for negative global imperfection amplitudes is even seen. Note that the VIPASA analysis predicts the panel to buckle between $250 \mathrm{lb} / \mathrm{in}$ and $350 \mathrm{lb} / \mathrm{in}$ indicating that the postbuckling strength of the panel is being accounted for in the nonlinear design. It is also important to notice that the locally postbuckled panel is insensitive to the local imperfections. Adding both global and local 


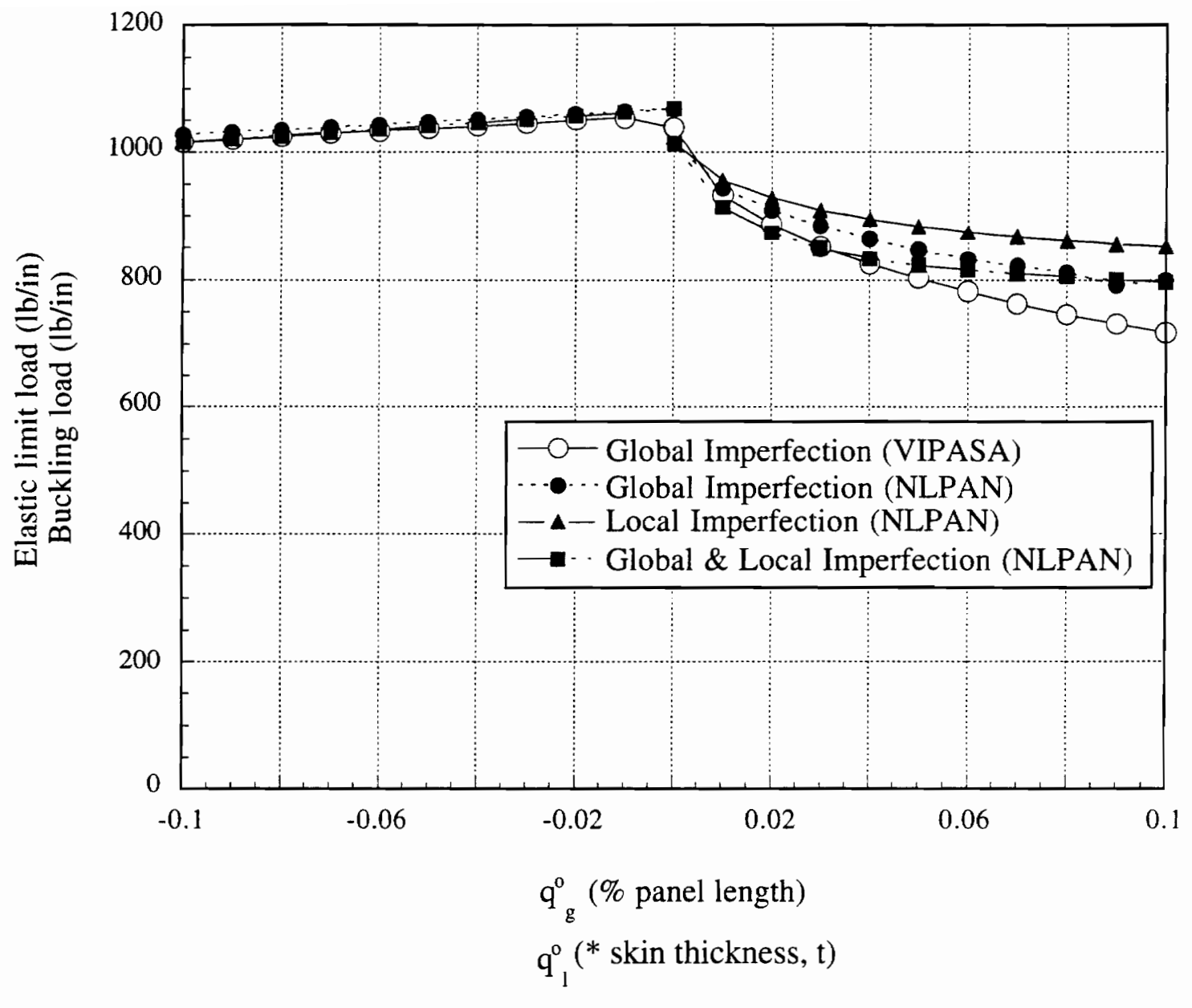

FIGURE 17. Imperfection sensitivity of the rounded-off PASCO design with no initial imperfections (Design load $=1000 \mathrm{lb} / \mathrm{in}$ ). 


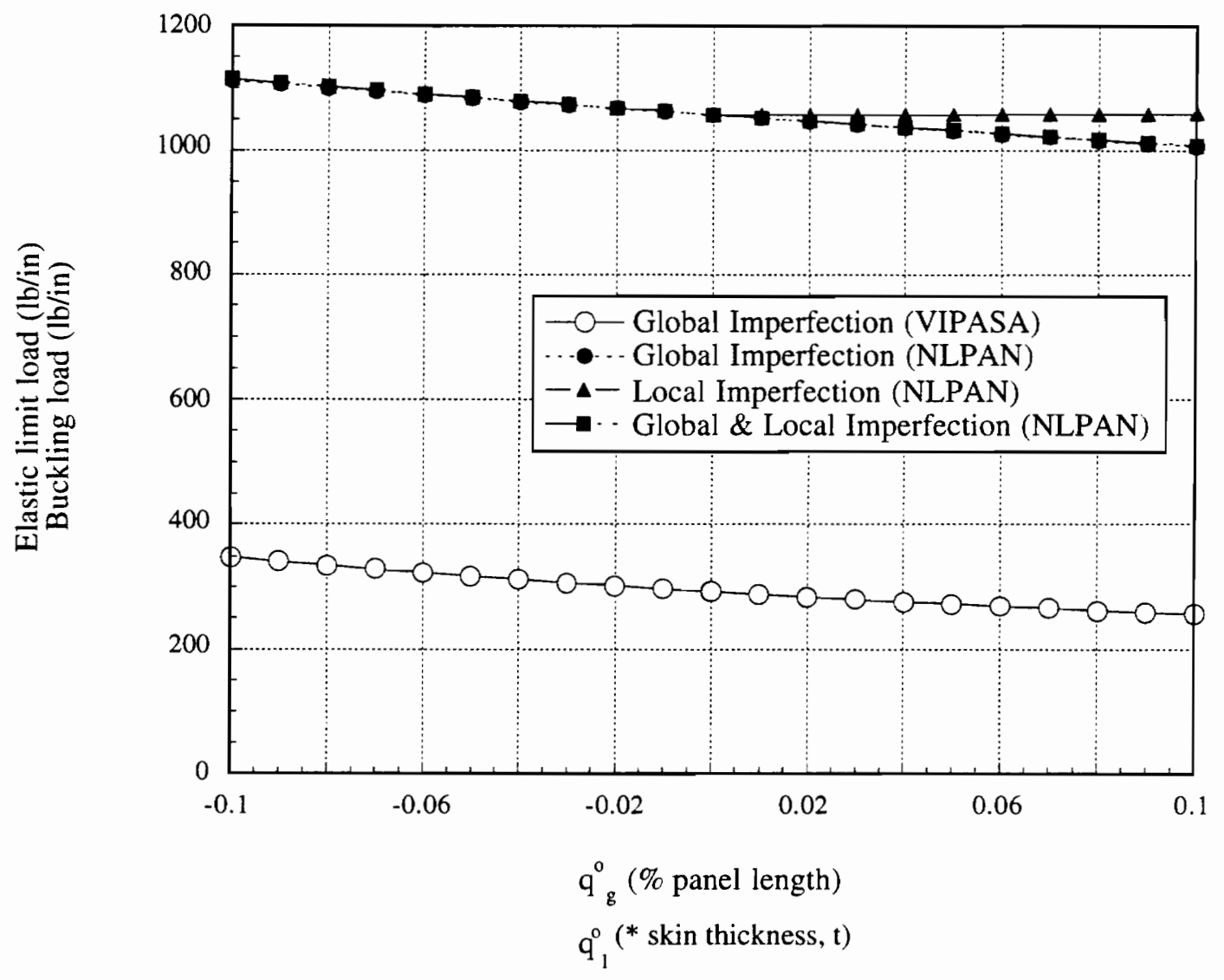

FIGURE 18. Imperfection sensitivity of the rounded-off NLPANOPT design with no initial imperfections (Design load $=1000 \mathrm{lb} / \mathrm{in}$ ). 
imperfections does not increase the panel's sensitivity but gives identical results to the case with only global imperfections.

It should be stated, however, that the previous designs carry the required load for the various imperfection amplitudes because they are rounded-off. The imperfection sensitivity of the continuous PASCO design with no initial imperfections (see Table 5) to global imperfection amplitudes is plotted in Figure 19. Both the VIPASA and NLPAN analysis predict the continuous PASCO design to be unable to carry the required load except for the imperfection it was designed and small negative amplitudes.

The imperfection sensitivity of the continuous and rounded-off NLPANOPT designs (see Table 5) to global imperfection amplitudes predicted by NLPAN is plotted in Figure 20. For the continuous design, the panel carries about $95 \%$ of the design load for the maximum positive imperfection amplitude, but for negative imperfection amplitudes greater than $0.02 \%$ of the panel length, the maximum load carried by the panel drops between $20 \%$ to $35 \%$ below the design load. At the maximum negative imperfection amplitude, the elastic limit load drops to $65 \%$ of the design load.

Next, we investigate the nature of the optimized designs that have built-in imperfections. Comparing the rounded-off designs obtained with an initial global imperfection of 0.0481 in. (see columns 3 and 5 of Table 5), the NLPANOPT design weighs $38 \%$ less than the PASCO design. The continuous PASCO design with an initial bowing imperfection is buckling load critical with the global eigenvalue increased by $48 \%$ above the critical value and the local eigenvalue held at the critical value. The sensitivity of the maximum load carried by the panel to a range of initial imperfections is shown in Figure 21 for the rounded-off PASCO design. The VIPASA analysis predicts the PASCO design to remain above the design load for all bowing imperfections. The NLPAN analyses predict the 


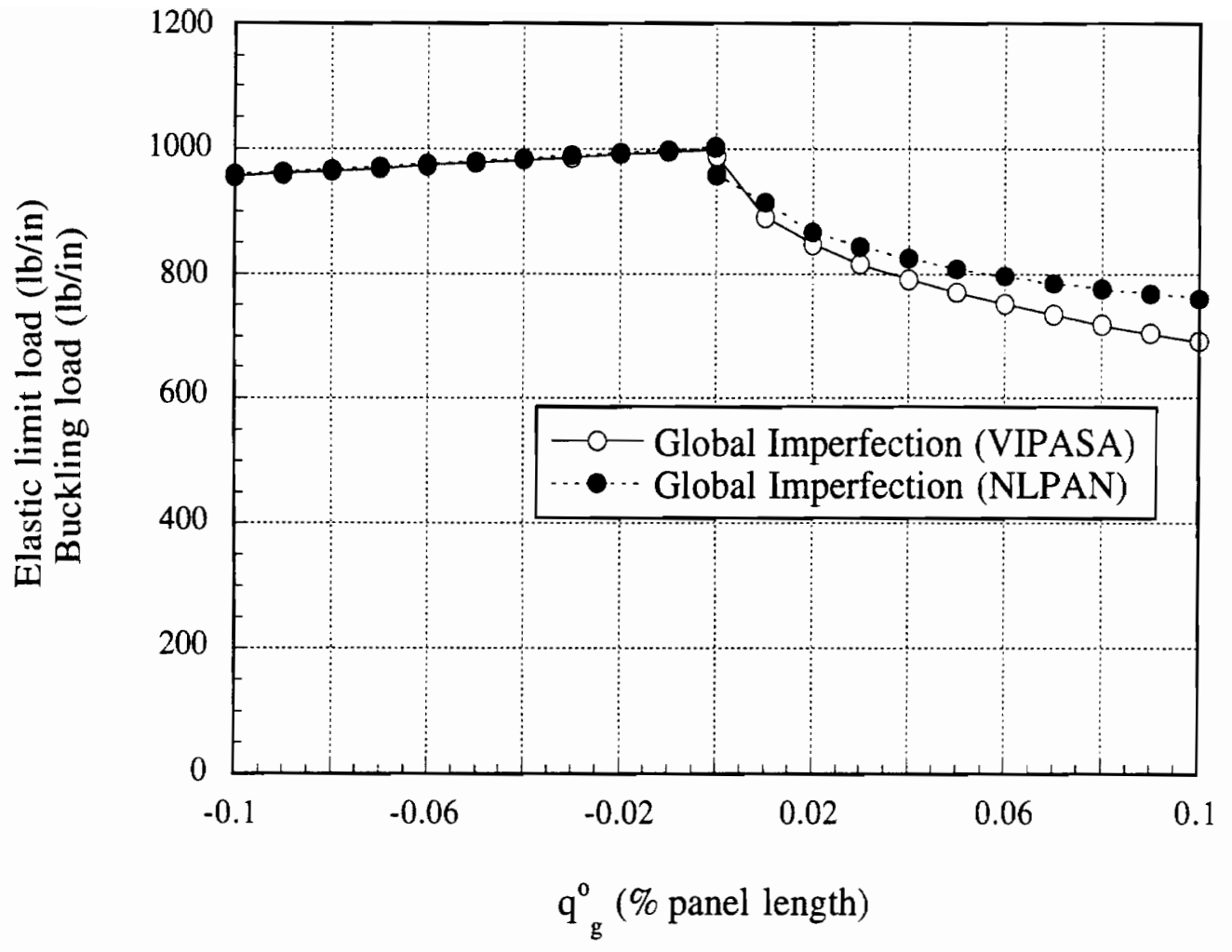

FIGURE 19. Imperfection sensitivity of the continuous PASCO design with no initial imperfections to global imperfection shapes (Design load = $1000 \mathrm{lb} / \mathrm{in})$. 


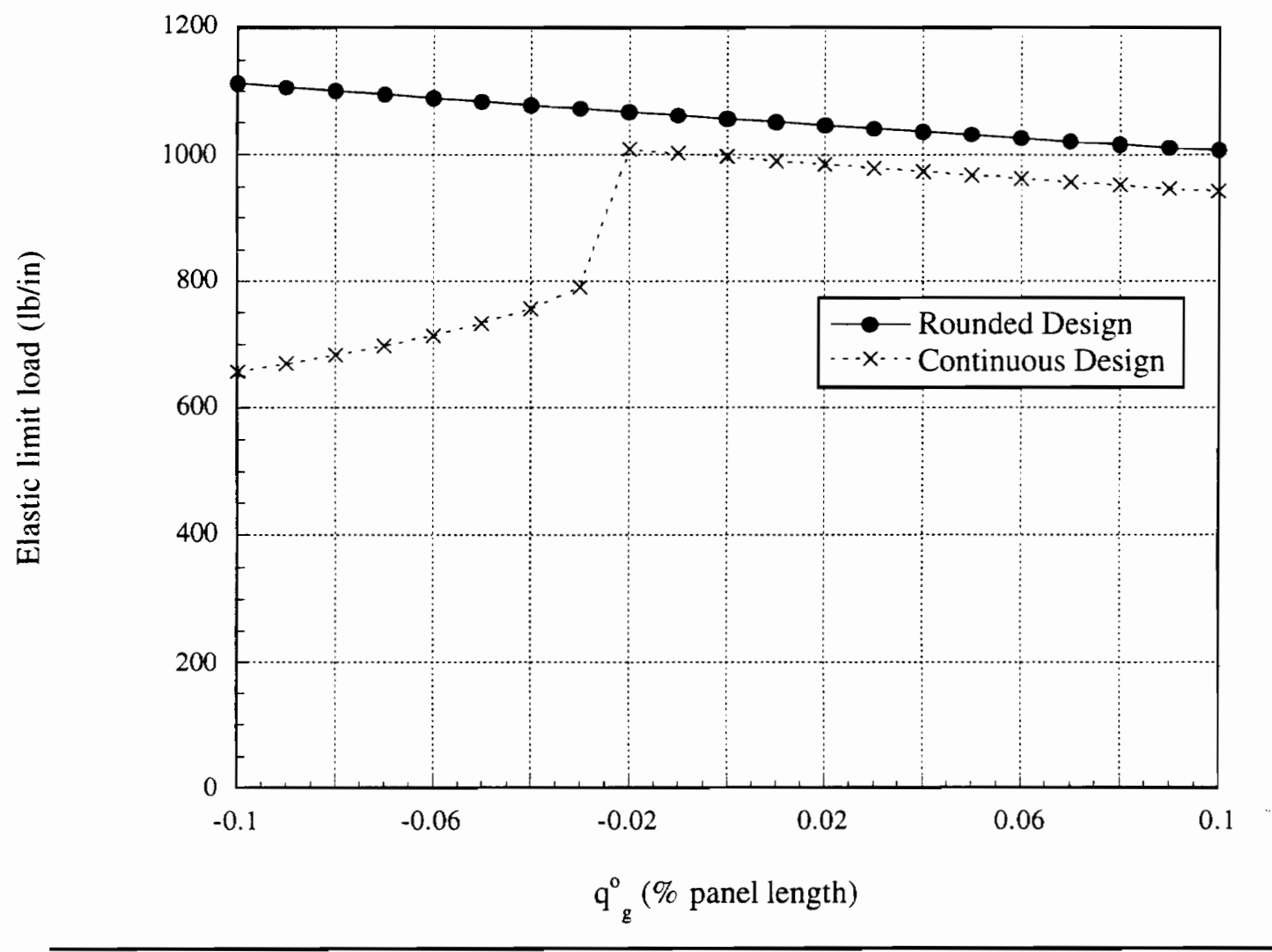

FIGURE 20. Comparison of imperfection sensitivity between the continuous and rounded-off NLPANOPT design with no imperfections using NLPAN (Design load $=1000 \mathrm{lb} / \mathrm{in}$ ). 


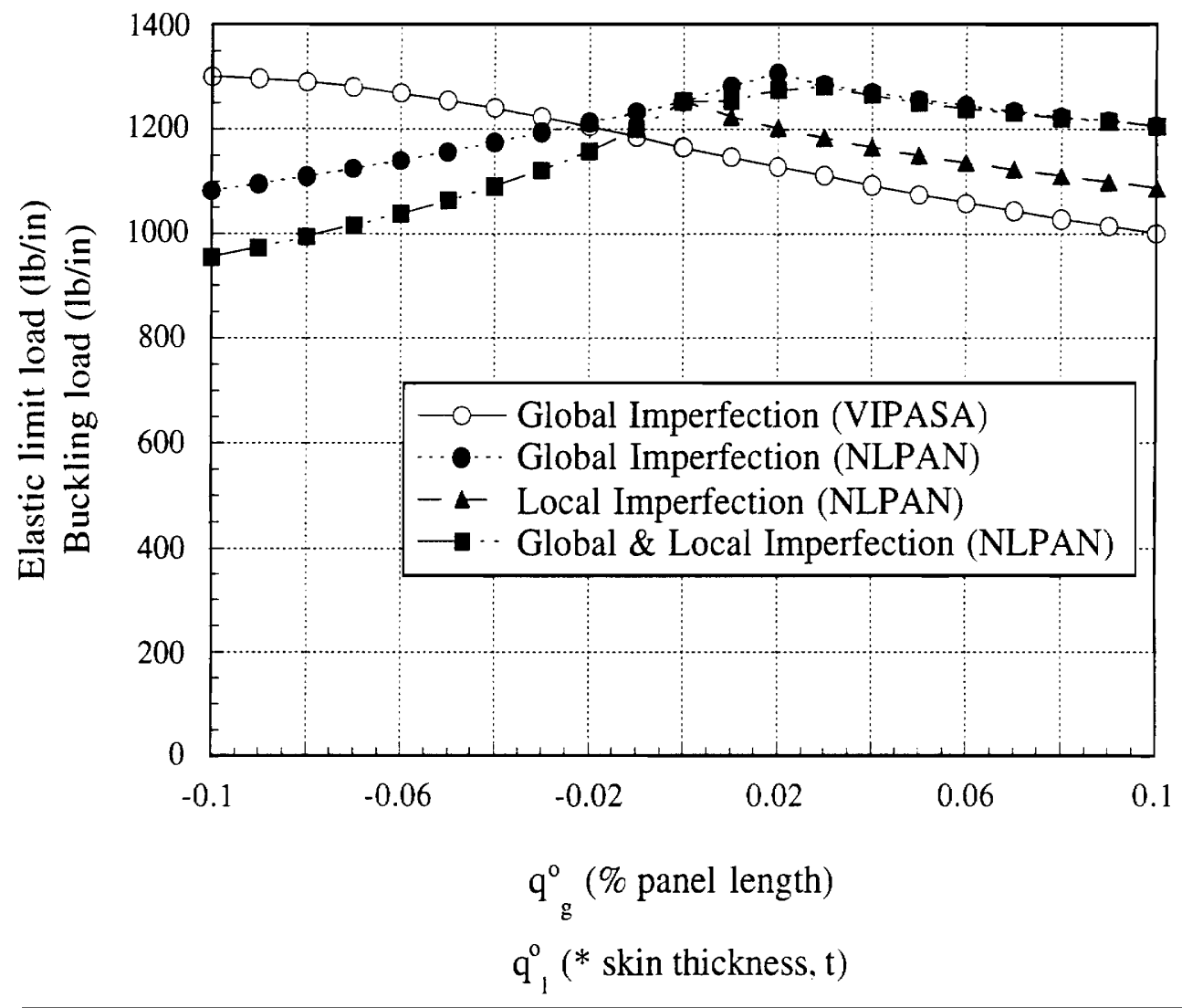

FIGURE 21. Imperfection sensitivity of the rounded-off PASCO design with initial bowing imperfection (Design load $=1000 \mathrm{lb} / \mathrm{in}$ ). 
panel to carry the required load for all global and local imperfection amplitudes separately, but for the combined imperfections greater than $-0.08 \%$ the panel cannot carry design load.

The continuous NLPANOPT design with an initial global imperfection is limit load critical and employs the postbuckling strength of the stiffened panel. The imperfection sensitivity of the rounded-off NLPANOPT design is shown in Figure 22. Note that since the rounded-off NLPANOPT designs with and without imperfections are almost the same, Figure 22 is almost identical to Figure 18. Both designs can carry the design load for all imperfection amplitudes and shapes. The imperfection sensitivity of the continuous NLPANOPT design with an initial global imperfection is shown in Figure 23. Unlike the results for the NLPANOPT design with no imperfections (see Figure 20), the continuous design carries the design load for all global imperfection amplitudes. Examining the continuous NLPANOPT panel designs in Table 5 , an increase in blade height and $0^{\circ}$ blade ply thickness decrease the imperfection sensitivity.

Another aspect about the final designs worth noting is the manner in which the design procedure handles the ply orientations. The stacking sequence is prescribed with lower bounds on the ply thickness design variables, $t_{\text {ply }}$. This approach prevents the elimination of specified ply orientations. Moreover, even if the lower bound was specified to be zero to eliminate a given orientation, the sequence of nonvanishing plies is still preassigned. Therefore, the designs may not be truly optimal. Also, no precautions were taken for restricting a large number of plies of the same orientation from being next to each other. For manufacturing, a restriction is usually implemented so no more than four plies of the same orientation may be next to each other. These shortcomings could be resolved easily by employing an optimizer such as a genetic algorithm capable of designing the stacking 


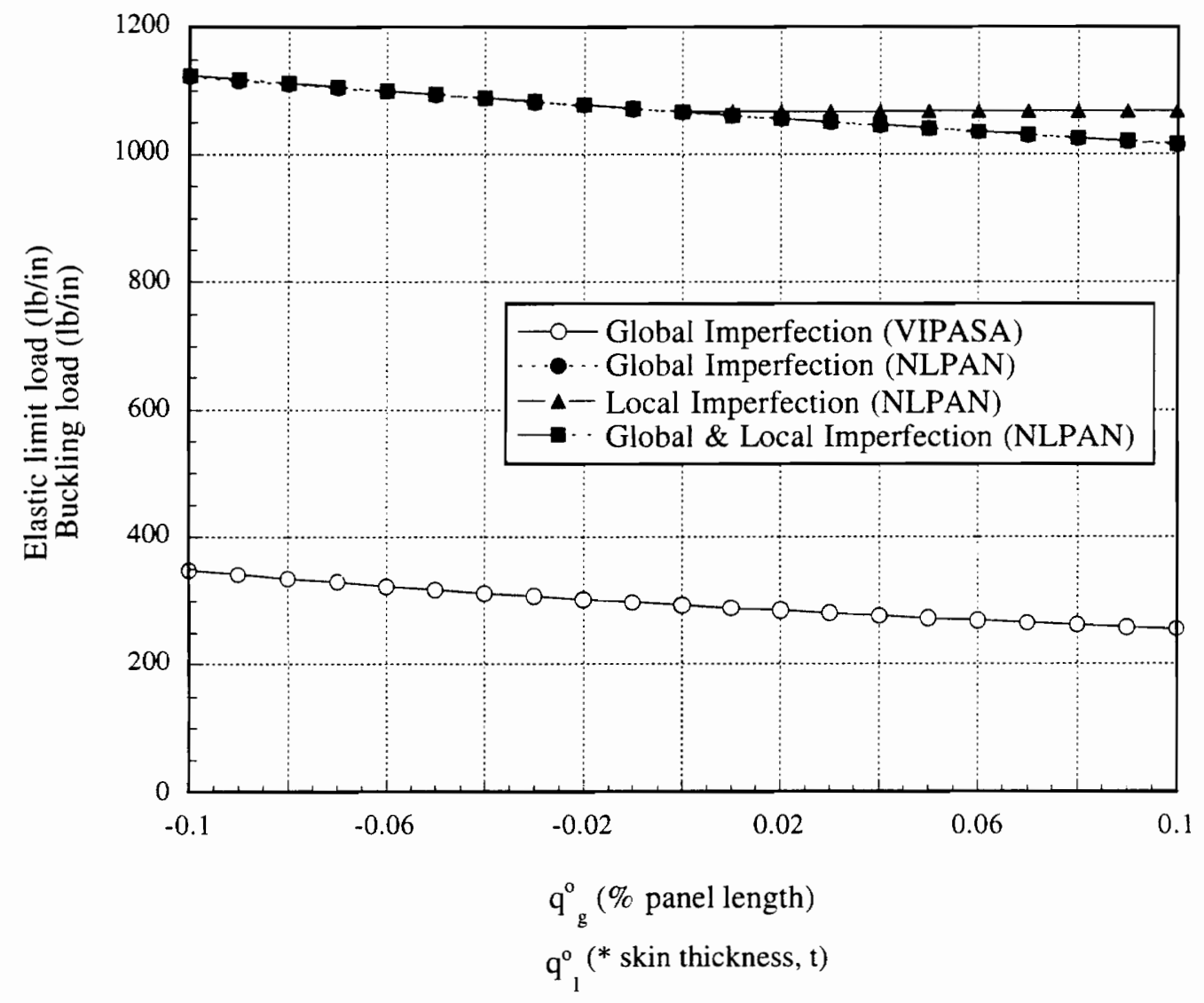

FIGURE 22. Imperfection sensitivity of the rounded-off NLPANOPT design with initial global imperfection shape $($ Design load $=1000 \mathrm{lb} / \mathrm{in})$. 


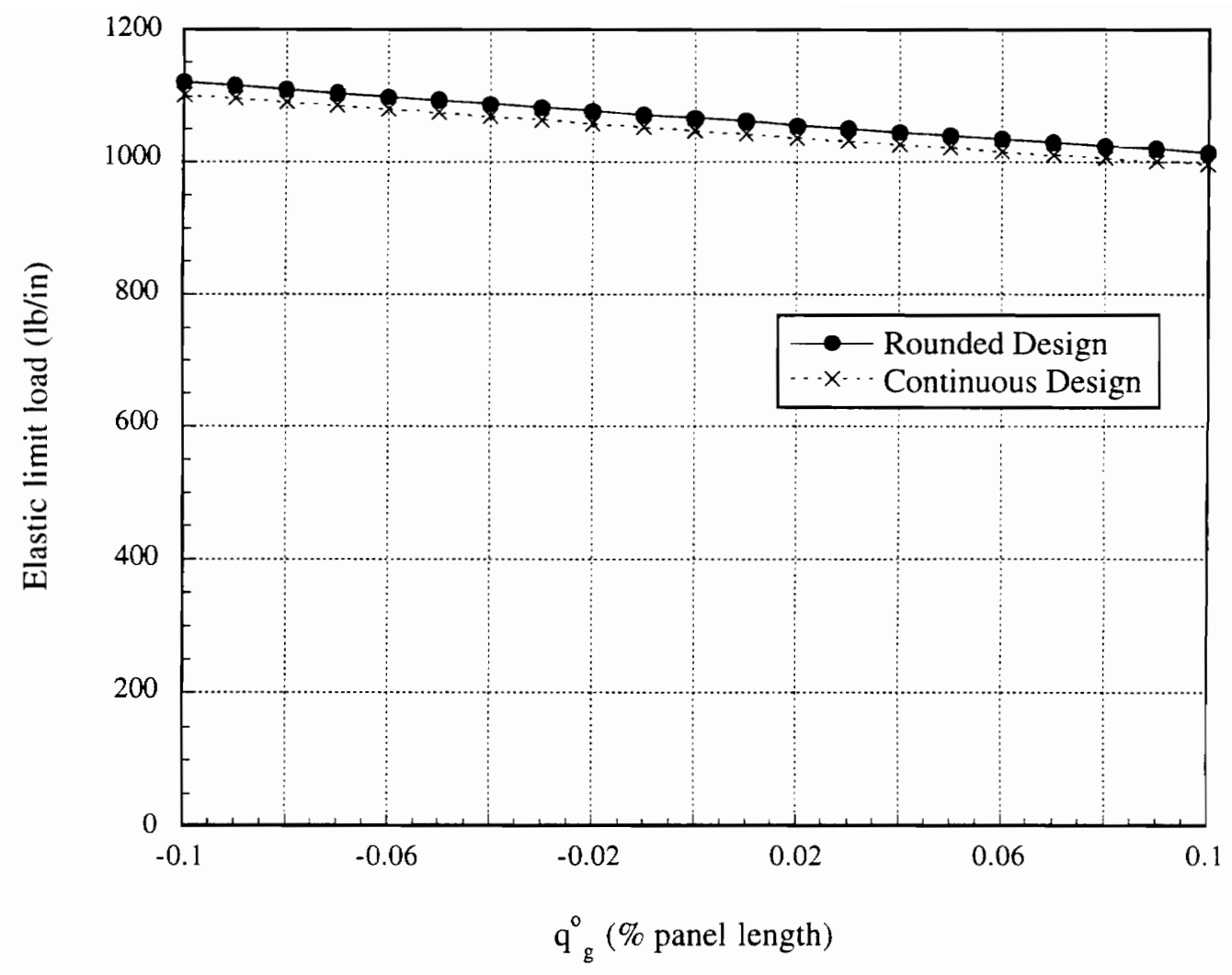

FIGURE 23. Comparison of imperfection sensitivity between the continuous and rounded-off NLPANOPT design with initial global imperfection shape using NLPAN (Design load $=1000 \mathrm{lb} / \mathrm{in}$ ). 
sequence [46]. Investigation of the effect of varying the skin laminate stacking sequence on the minimum-weight design will be discussed in Section 4.2 for this axial compressive load.

\subsubsection{Case 3: $5000 \mathrm{lb} /$ in Design Load}

The rounded-off design results for the $5000 \mathrm{lb} / \mathrm{in}$ axial compressive load are listed in Table

6 . The numbers in parentheses for the $\lambda_{\text {global }}$ row represent the global eigenvalues for the symmetric global mode (see Section 2.2 for a discussion of symmetric vs. antisymmetric global mode). The NLPANOPT designs contain an antisymmetric primary global mode.

Table 6: Summary of panel designs for $N_{x}=5000 \mathrm{lb} / \mathrm{in}$.

\begin{tabular}{|c|c|c|c|c|}
\hline & PASCO & $\begin{array}{c}\text { PASCO } / \\
\text { imperfection }\end{array}$ & NLPANOPT & $\begin{array}{c}\text { NLPANOPT } \\
\text { w/ } \\
\text { imperfection }\end{array}$ \\
\hline \hline $\mathrm{b}$ (in.) & 2.16810 & 2.50539 & 2.78078 & 2.74207 \\
\hline $\mathrm{t}_{1}\left(0^{\circ}{ }_{\text {skin }}\right)$ & 1 & 1 & 1 & 1 \\
\hline $\mathrm{t}_{2}\left(90^{\circ}{ }_{\text {skin }}\right)$ & 1 & 1 & 1 & 1 \\
\hline $\mathrm{t}_{3}\left( \pm 45^{\circ}{ }_{\text {skin }}\right)$ & 4 & 4 & 1 & 1 \\
\hline $\mathrm{t}_{4}\left(0^{\circ}{ }_{\text {blade }}\right)$ & 19 & 16 & 10 & 13 \\
\hline $\mathrm{t}_{5}\left(90^{\circ}{ }_{\text {blade }}\right)$ & 1 & 1 & 3 & 1 \\
\hline $\mathrm{t}_{6}\left( \pm 45^{\circ}{ }_{\text {blade }}\right)$ & 1 & 2 & 2 & 3 \\
\hline \begin{tabular}{c} 
Unit Weight $_{(\text {lbs. })}$ \\
\hline$\lambda_{\text {global }}$
\end{tabular} & 3.6365 & 3.7982 & 2.4737 & 2.6312 \\
\hline$\lambda_{\text {local }}$ & 1.05 & 1.41 & $0.665(1.09)$ & $0.817(1.23)$ \\
\hline $\mathrm{m}_{\text {local }}$ & 5 & 1.0 & 0.124 & 0.145 \\
\hline
\end{tabular}

For the designs with no imperfections, the rounded-off NLPANOPT design weighs $32 \%$ less than the rounded-off PASCO design. The continuous PASCO design is buckling load 
critical with simultaneous global and local buckling modes. The continuous NLPANOPT design is limit load critical and is in a locally postbuckled state at the design load. The imperfection sensitivity of the rounded-off PASCO and NLPANOPT designs, evaluated using both linear and nonlinear analyses, is shown in Figure 24 and Figure 25, respectively.

In Figure 24, imperfection amplitudes greater than $0.02 \%$ of the panel length for both positive and negative directions reduce the buckling load of the PASCO design below the design load. The nonlinear analysis predicts the panel to be incapable of carrying the design load for the entire range of imperfection amplitudes and shapes with the largest decrease in load of $20 \%$ for a global and local amplitude of -0.1 .

The NLPANOPT design (Figure 25) cannot carry the required load for an initial global imperfection amplitude above $0.06 \%$ of the panel length for either direction. The largest decrease in load is $5 \%$ for a global imperfection amplitude of $0.1 \%$ of the panel length. Local imperfections do not effect the response of the panel and the combined imperfections give identical results to the global imperfection case. The VIPASA analysis supplies the buckling load of the NLPANOPT design proving the postbuckled state of the panel.

The NLPANOPT design listed in Table 6 is the minimum-weight design out of a number of designs obtained from various initial design configurations. A number of local optima, all limit load critical, were detected and are listed in Table 7 (including the design listed in Table 6 as Design 1).The weight trends for these three designs are shown graphically in Figure 26. As the total weight increases from Design 1 to Design 3, the majority of the weight switches from the blade to the skin, thus switching from local to global postbuckling behavior. This is also seen in the load versus end shortening response for each of the three designs shown in Figure 27. The initial axial stiffness increases from Design 1 to 


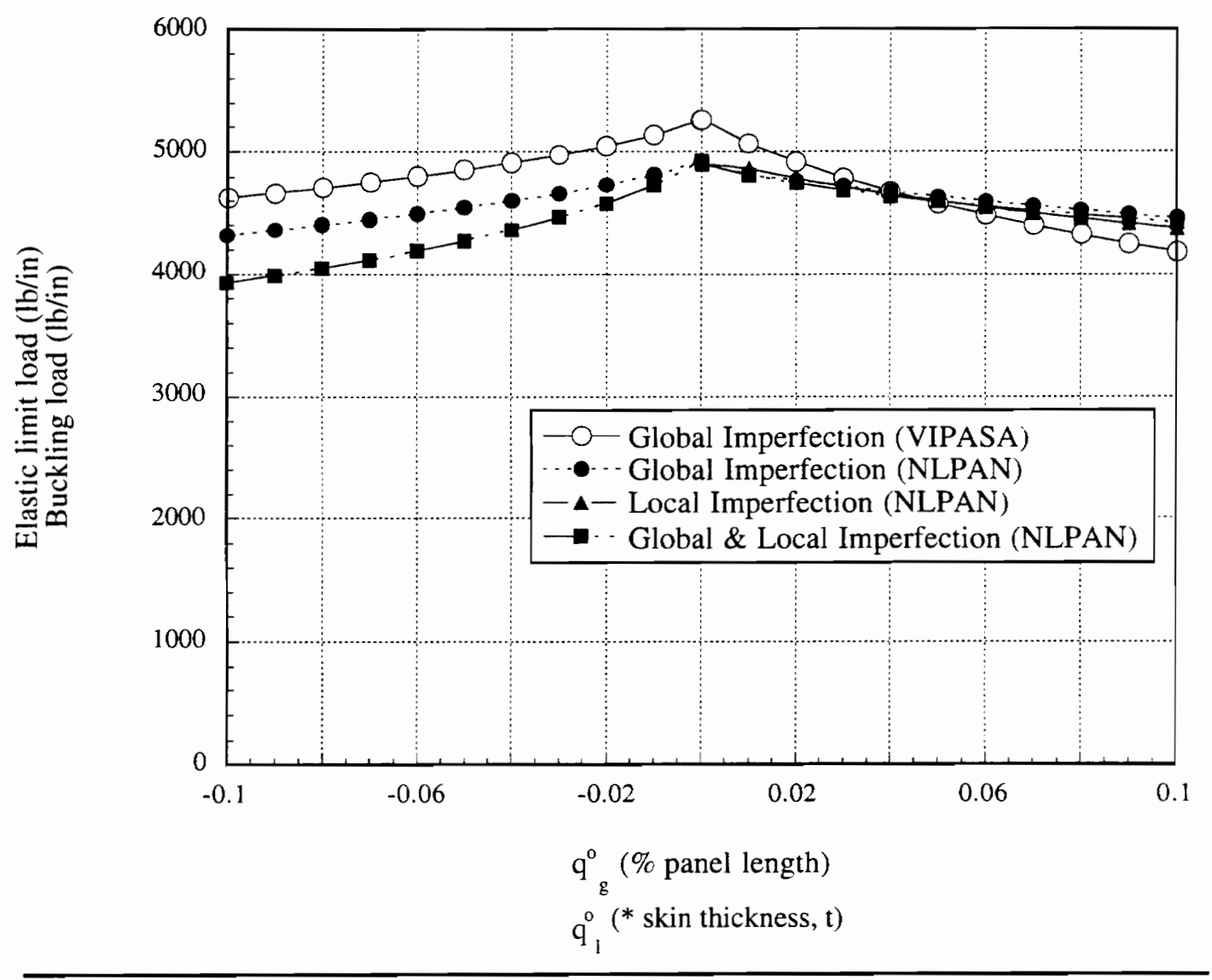

FIGURE 24. Imperfection sensitivity of PASCO design with no initial imperfections $($ Design load $=5000 \mathrm{lb} / \mathrm{in})$. 


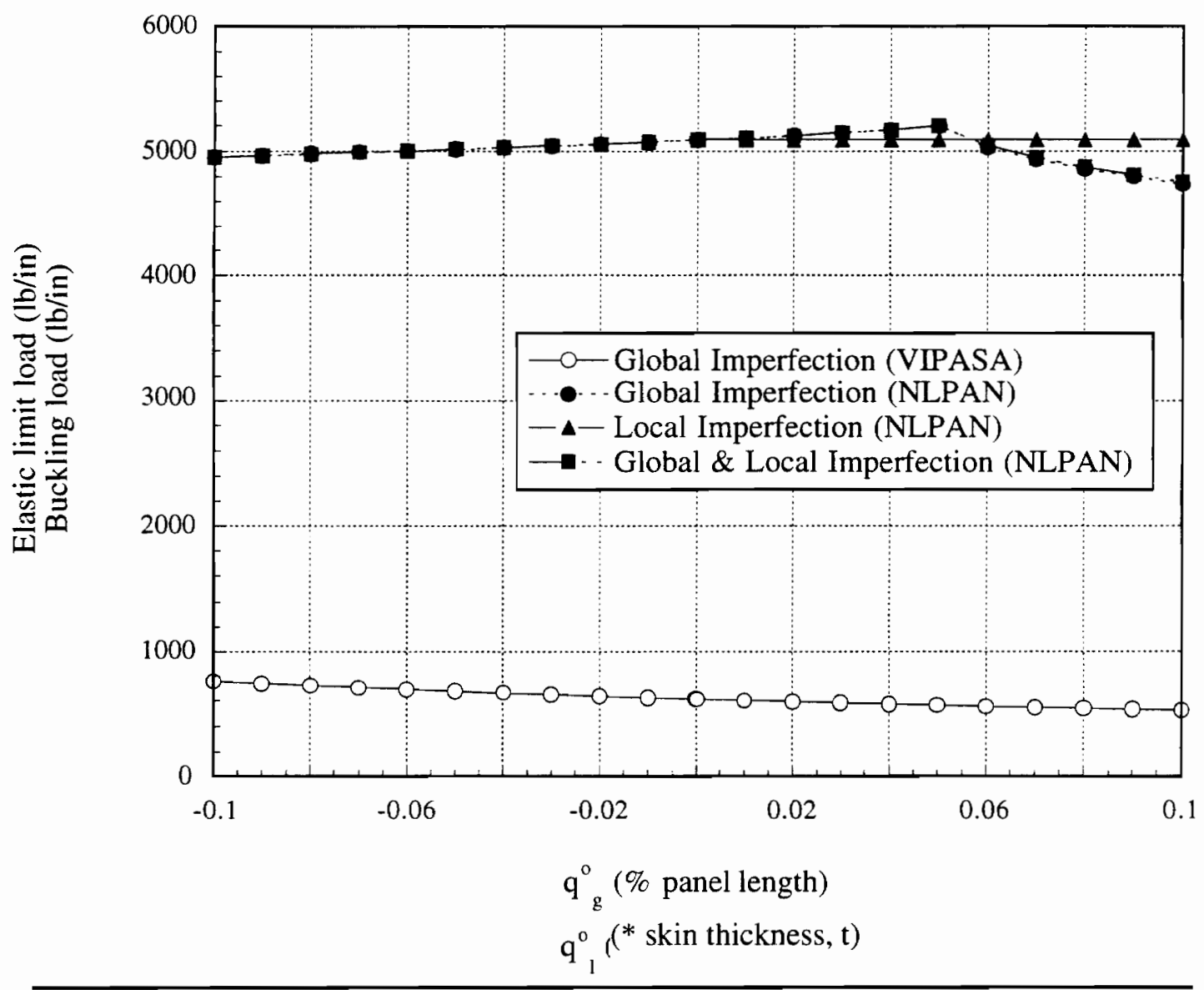

FIGURE 25. Imperfection sensitivity of NLPANOPT design with no initial imperfections (Design load $=\mathbf{5 0 0 0} \mathrm{lb} / \mathrm{in})$. 


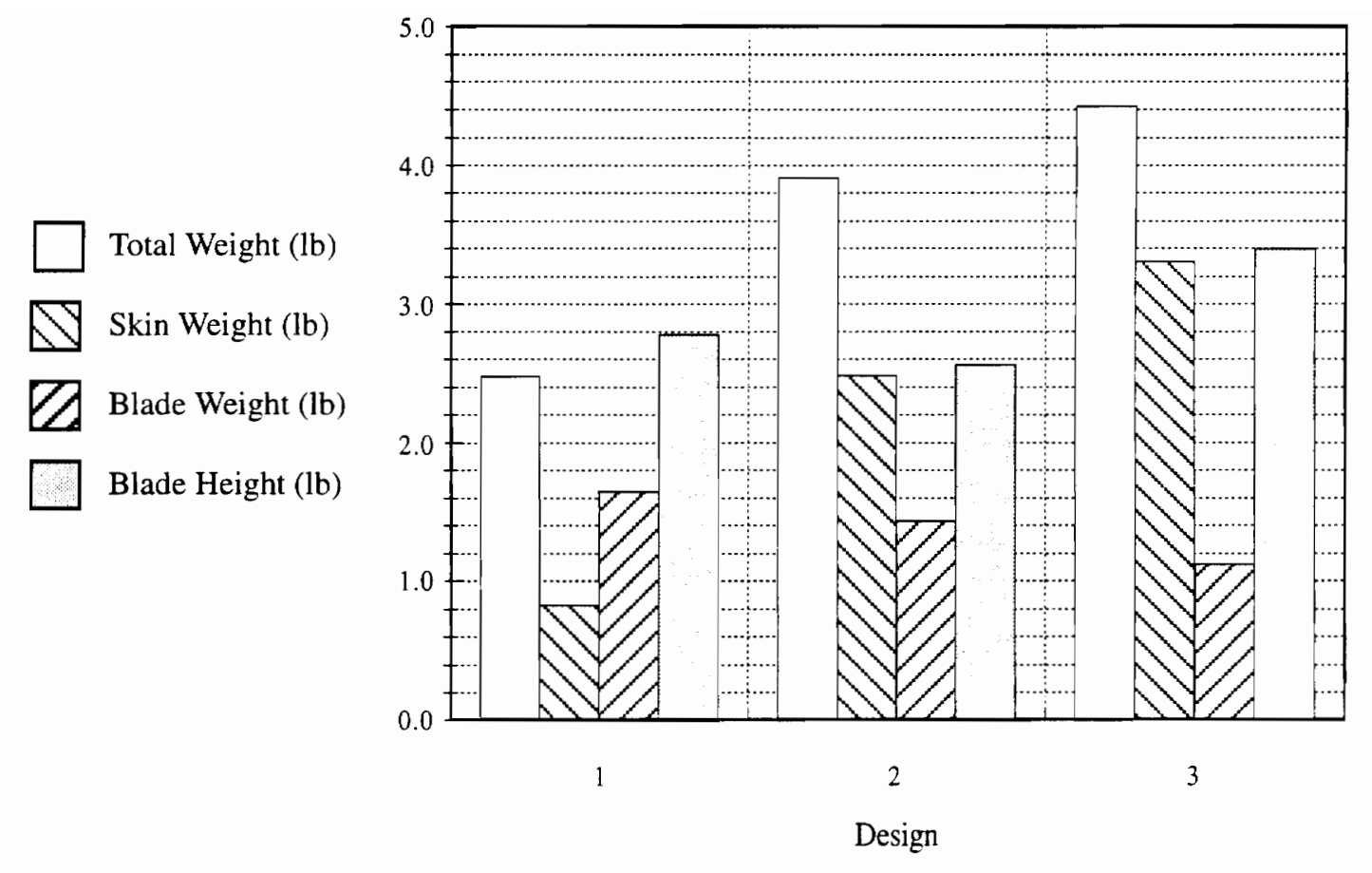

FIGURE 26. Weight and blade height trends of multiple optima for NLPANOPT designs with no initial imperfections (Design load $=5000 \mathrm{lb} / \mathrm{in})$. 


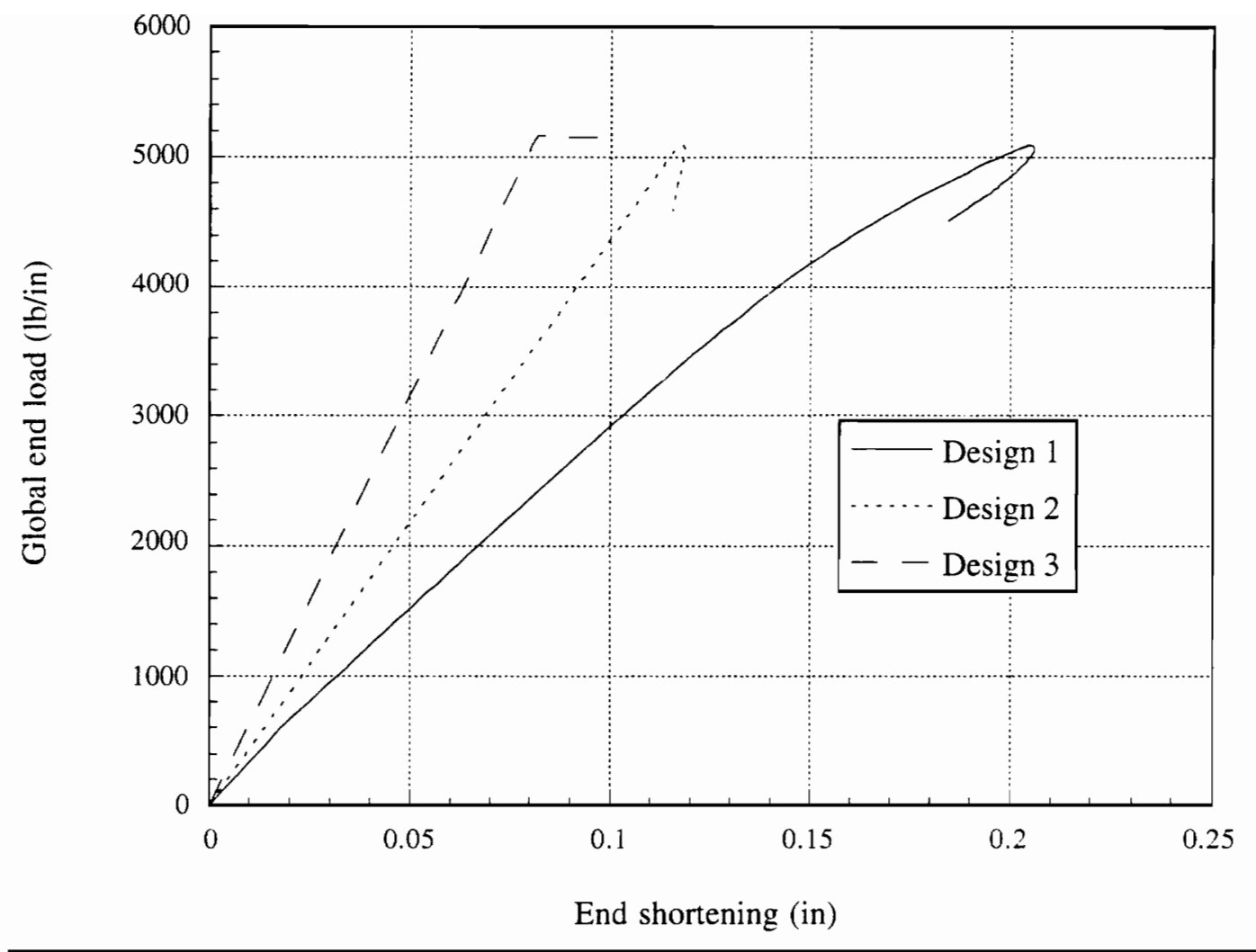

FIGURE 27. Panel response of multiple optima for NLPANOPT designs with no initial imperfections (Design load $=\mathbf{5 0 0 0} \mathrm{lb} / \mathrm{in}$ ). 
Design 3. Design 1 buckles locally around $600 \mathrm{lb} /$ in followed by a decrease in axial stiffness. With the arrival of the symmetric global buckling load, the panel loses it load-carrying capabilities. Design 2 buckles locally around $5100 \mathrm{lb} /$ in followed by a dramatic decrease in load-carrying capability. Design 3 buckles globally around $5200 \mathrm{lb} / \mathrm{in}$ followed by a neutral load-carrying capability. Referring back to Figure 26, the blade height is reduced by $8 \%$ for Design 2 compared to Design 1 and is increased by $22 \%$ for Design 3 compared to Design 1.

Table 7: Summary of NLPANOPT designs with no initial imperfections for $\mathrm{N}_{x}=\mathbf{5 0 0 0}$ lb/in.

\begin{tabular}{|c|c|c|c|}
\hline & Design 1 & Design 2 & Design 3 \\
\hline \hline $\mathrm{b}$ (in.) & 2.78078 & 2.55935 & 3.39899 \\
\hline $\mathrm{t}_{1}\left(0^{\circ}{ }_{\text {skin }}\right)$ & 1 & 3 & 10 \\
\hline $\mathrm{t}_{2}\left(90^{\circ}{ }_{\text {skin }}\right)$ & 1 & 3 & 2 \\
\hline $\mathrm{t}_{3}\left( \pm 45^{\circ}\right.$ skin $)$ & 1 & 3 & 2 \\
\hline $\mathrm{t}_{4}\left(0^{\circ}{ }_{\text {blade }}\right)$ & 10 & 8 & 1 \\
\hline $\mathrm{t}_{5}\left(90^{\circ}{ }_{\text {blade }}\right)$ & 3 & 3 & 3 \\
\hline $\mathrm{t}_{6}\left( \pm 45^{\circ}{ }_{\text {blade }}\right)$ & 2 & 3 & 3 \\
\hline Unit Weight $(\mathrm{lbs})$. & 2.47368 & 3.91209 & 4.42579 \\
\hline$\lambda_{\text {global }}$ & $0.665(1.09)$ & 1.14 & 1.04 \\
\hline$\lambda_{\text {local }}$ & 0.124 & 1.02 & 1.09 \\
\hline $\mathrm{m}_{\text {local }}$ & 12 & 7 & 7 \\
\hline
\end{tabular}

The imperfection sensitivity of Design 2 and Design 3 is shown in Figure 28 and Figure 29 , respectively. The imperfection sensitivity of Design 2 is similar to the response of the PASCO design (see Figure 24) for this design load. The buckling load is reduced below the design load for positive bowing imperfections greater than $0.01 \%$ of the panel length and for negative bowing imperfections greater than $-0.03 \%$ of the panel length. The 


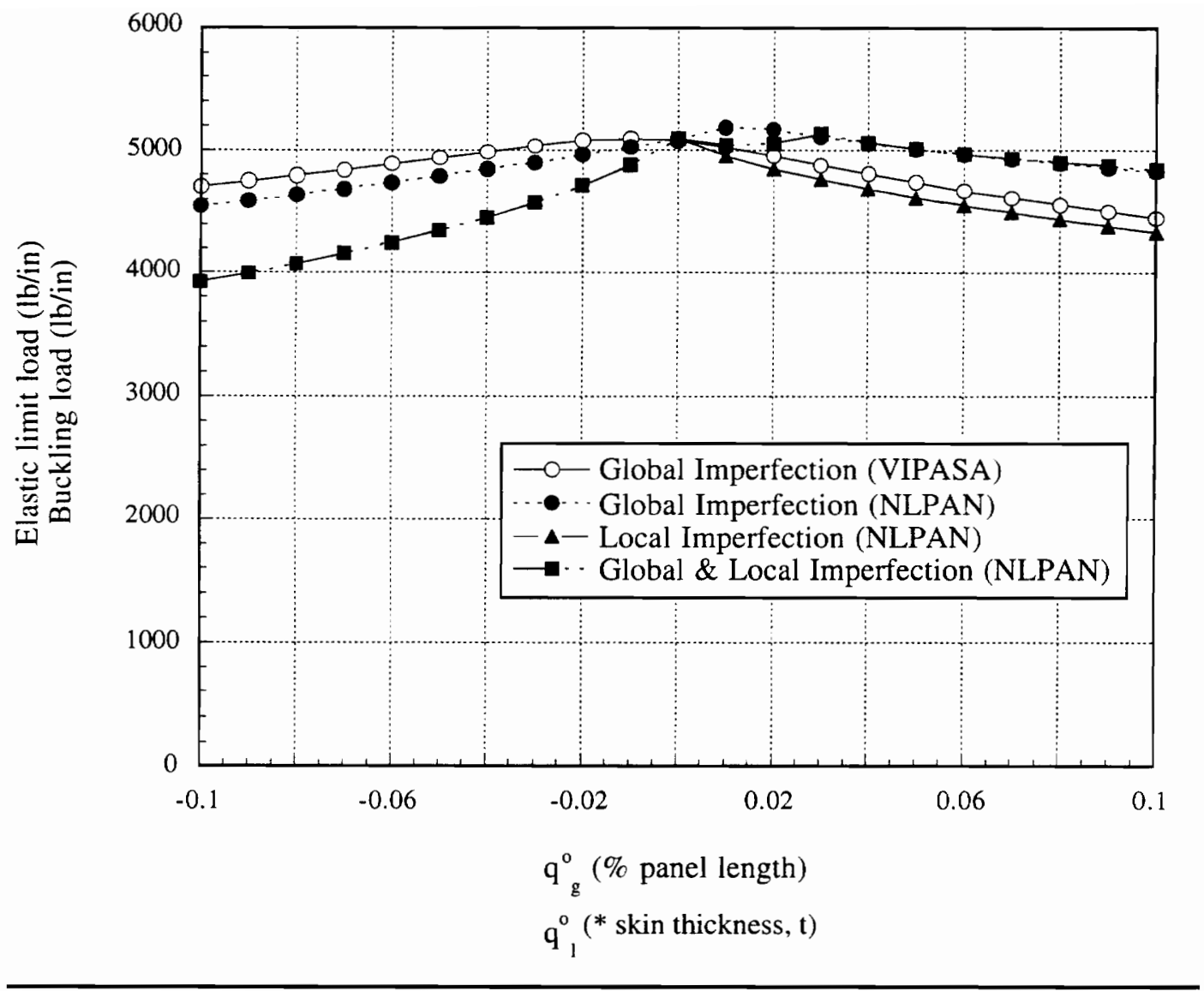

FIGURE 28. Imperfection sensitivity of Design 2 obtained from NLPANOPT design with no initial imperfections $($ Design load $=5000 \mathrm{lb} / \mathrm{in})$. 


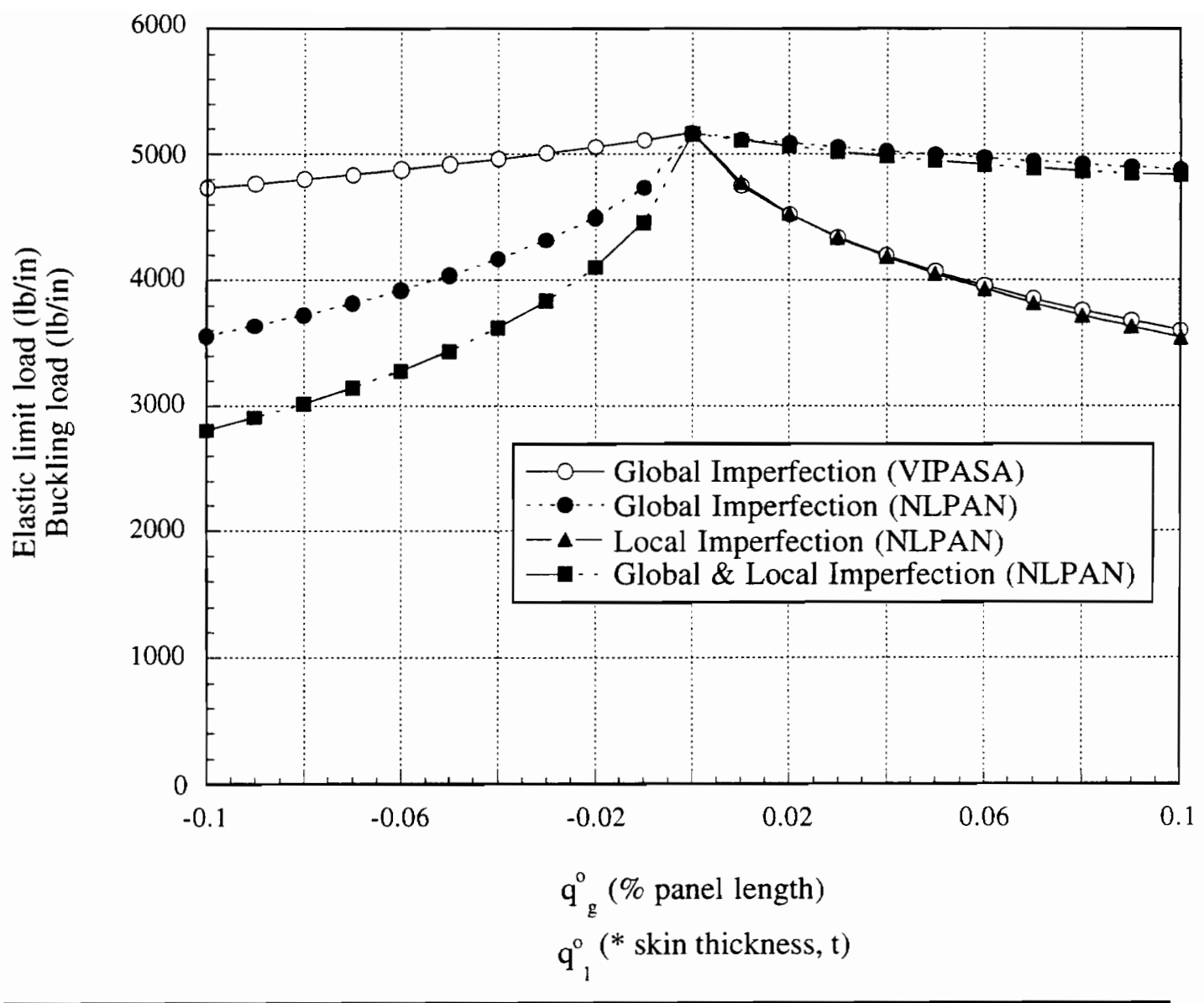

FIGURE 29. Imperfection sensitivity of Design 3 obtained from NLPANOPT design with no initial imperfections (Design load $=5000 \mathrm{lb} / \mathrm{in}$ ). 
NLPAN analysis predicts small positive global imperfections to increase the maximum load carried by the panel above the load predicted for no imperfections. As the positive global imperfections increase to $0.03 \%$ of the panel length, however, the maximum load begins to decrease until it drops below the design load for a global imperfection amplitude of $.06 \%$ of the panel length. For negative global imperfection amplitudes, the maximum load drops immediately until a value of $4550 \mathrm{lb} / \mathrm{in}$ is reached at an amplitude of $-0.1 \%$ of the panel length. The panel is sensitive to local imperfections with the maximum load decreasing as the amplitude increases. For the combined imperfections, the local imperfections reduce the positive effect seen for the global amplitudes at $+0.01 \%$ and $+0.02 \%$ of the panel length. As the amplitude increases, though, the global imperfection dominates the behavior and the results are identical to the global imperfection case. For negative global imperfection amplitudes, the local imperfections reduce the maximum load compared to the global imperfection case.

For Design 3 (Figure 29) the buckling load drops quickly with the addition of positive bowing imperfection amplitudes. The same occurs for the negative bowing imperfection amplitudes, but not as quickly. The opposite effect is seen for the nonlinear analysis with global imperfection amplitudes. The panel becomes more sensitive to the negative global imperfection amplitudes. The panel is sensitive to local imperfection amplitudes and reaches its maximum load at or very near the buckling load. This sensitivity reduces the maximum load slightly with the addition of positive global imperfection amplitudes and even more with the addition of negative global imperfection amplitudes.

Based on these observations, it can be concluded that even though a number of local optimum were detected by NLPANOPT, the minimum-weight design was the least sensitive (see Figure 25) to imperfections due to its local postbuckling behavior. 
Comparing the rounded-off designs obtained by including an initial global imperfection (third and fifth columns of Table 6), the NLPANOPT design weighs $31 \%$ less than the PASCO design. The continuous PASCO design is buckling load critical, while the continuous NLPANOPT design is critical for the shear strain material constraint. The imperfection sensitivity of the rounded-off PASCO design is shown in Figure 30 and the roundedoff NLPANOPT design is shown in Figure 31. With a $4.5 \%$ increase in weight compared to the PASCO design with no imperfections, the PASCO design buckles above the required load for all bowing imperfections. The NLPAN analyses predict the panel to be incapable of carrying the design load for large local imperfections, for large negative global imperfection, and for all combined local imperfection and negative global imperfection. The NLPANOPT design is $6 \%$ heavier than the NLPANOPT design without initial imperfections. The panel carries the design load for all imperfection amplitudes and shapes with local postbuckling behavior.

Similar to the NLPANOPT design with no initial imperfections, more than one optimum was detected for the NLPANOPT design with an initial global imperfection. Table 8 lists the two designs detected that displayed different panel responses (Design 1 is the design listed in Table 6). The continuous Design 2 is also critical for the shear strain material constraint. The load versus end shortening panel response for each of the two designs is shown in Figure 32. Design 1 buckles locally and has a slight loss of axial stiffness around $700 \mathrm{lb} / \mathrm{in}$, while the axial stiffness loss for Design 2 does not occur until $4500 \mathrm{lb} / \mathrm{in}$ where it buckles locally. Both designs have local postbuckling behavior, but Design 1 reaches much further into the postbuckling region and has a 50\% weight savings compared to Design 2. The imperfection sensitivity of Design 2 is shown in Figure 33. Comparing Figure 31 and Figure 33, both designs remain above the design load for the entire range of imperfections. The total change in elastic limit load from the negative to positive imper- 


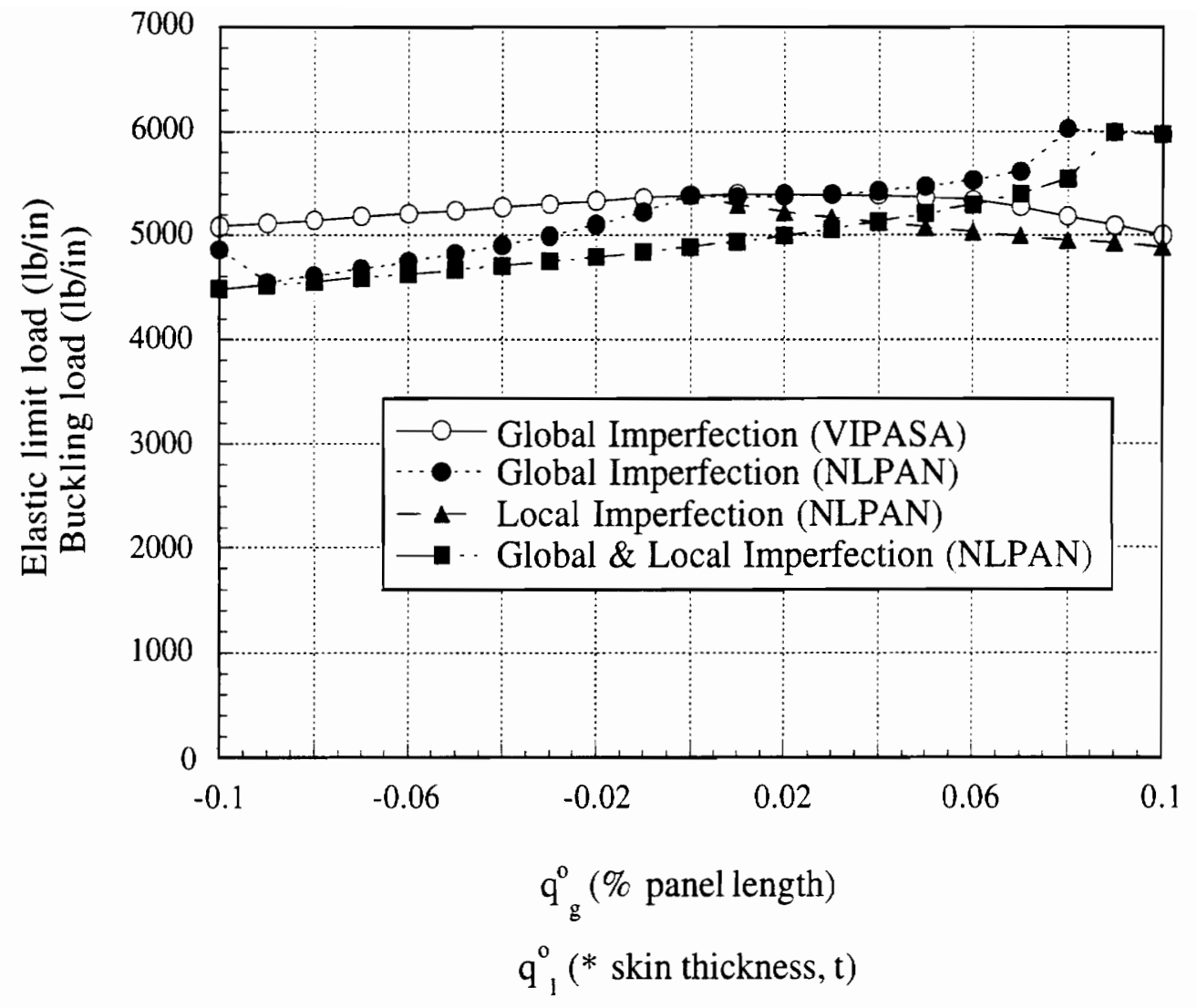

FIGURE 30. Imperfection sensitivity of PASCO design with initial bowing imperfection (Design load $=5000 \mathrm{lb} / \mathrm{in})$. 


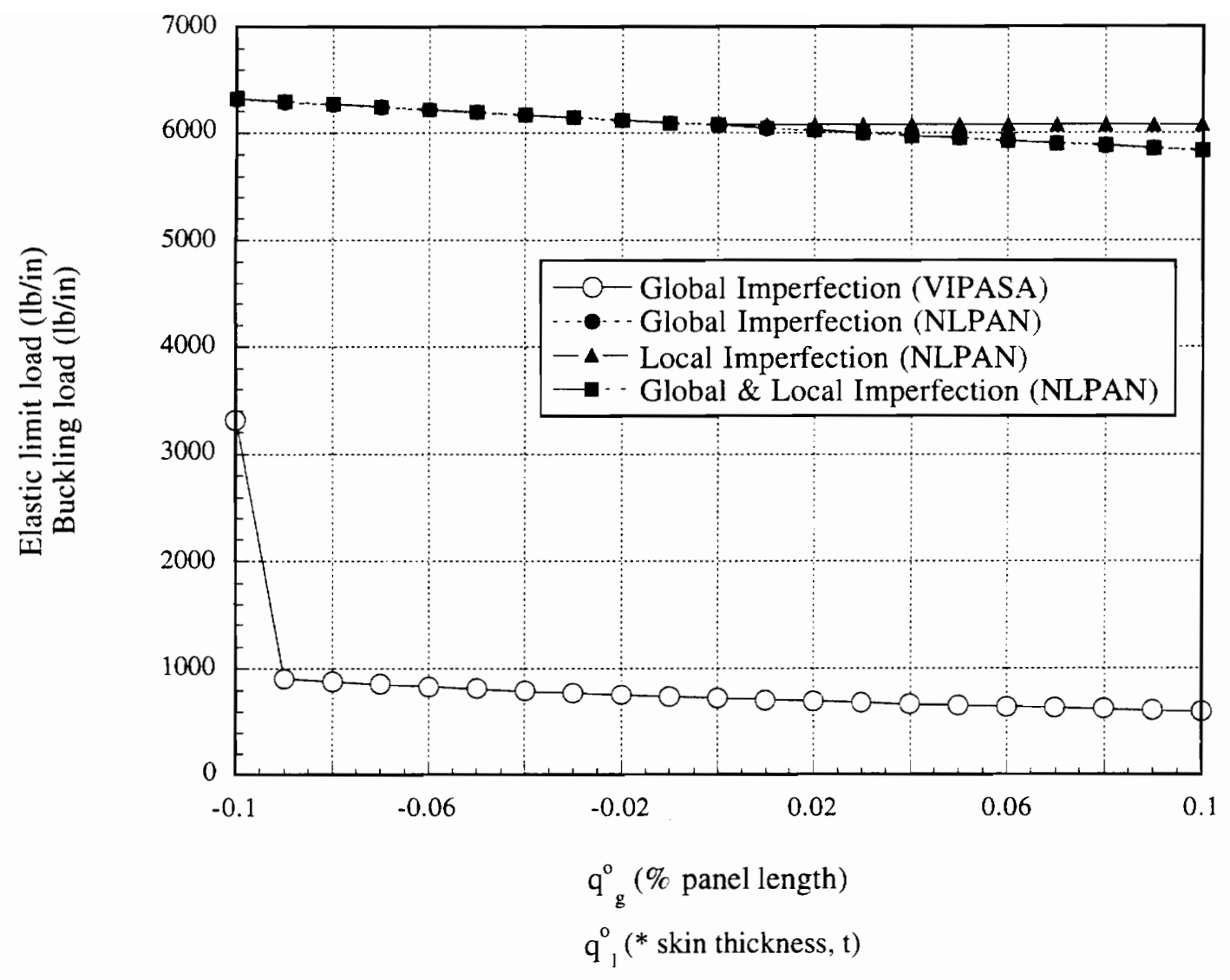

FIGURE 31. Imperfection sensitivity of NLPANOPT design with initial global imperfection shape (Design load $=5000 \mathrm{lb} / \mathrm{in})$. 


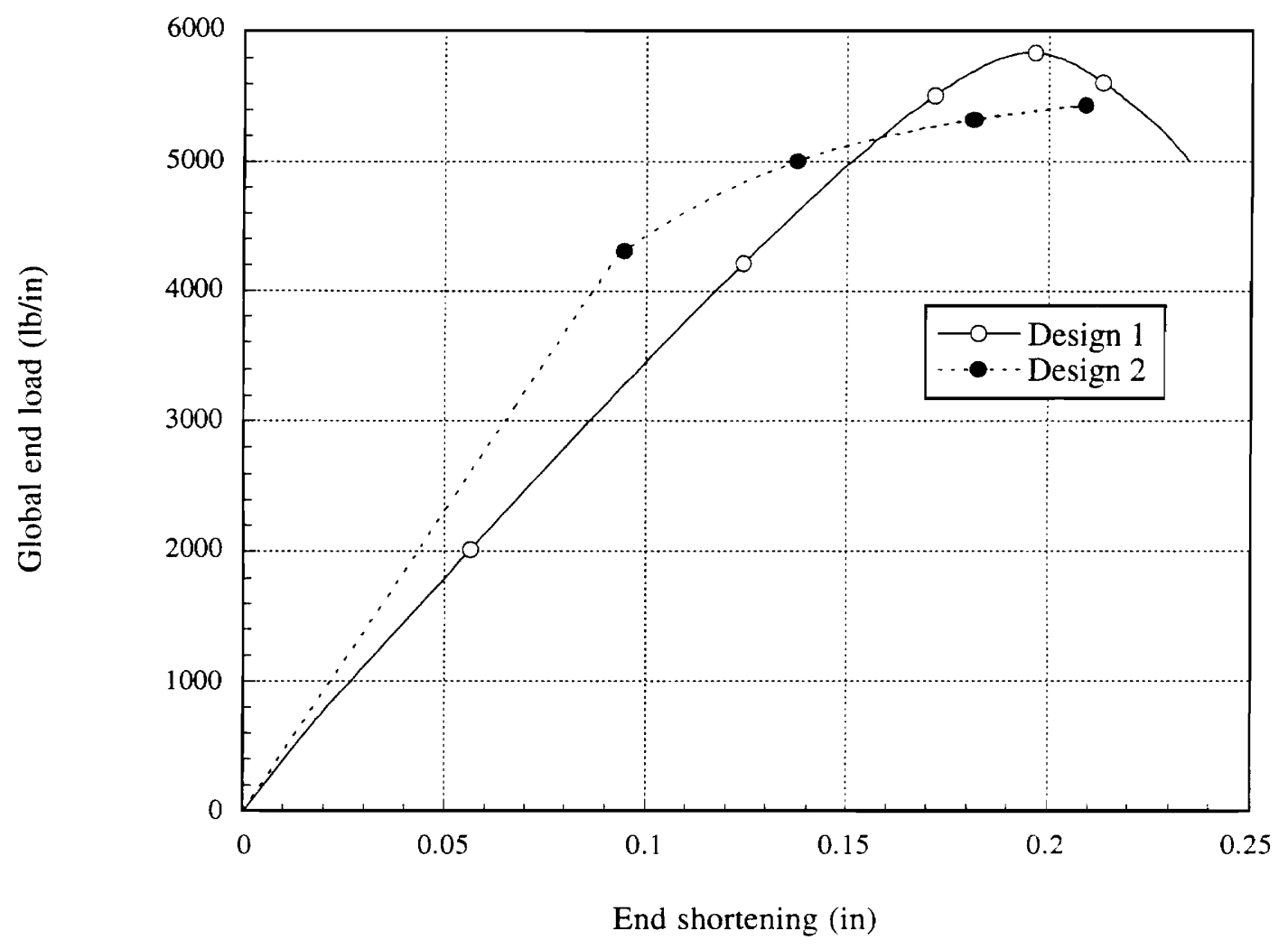

FIGURE 32. Panel response for multiple optima of NLPANOPT designs with initial global imperfection shape (Design load $=5000 \mathrm{lb} / \mathrm{in}$ ). 


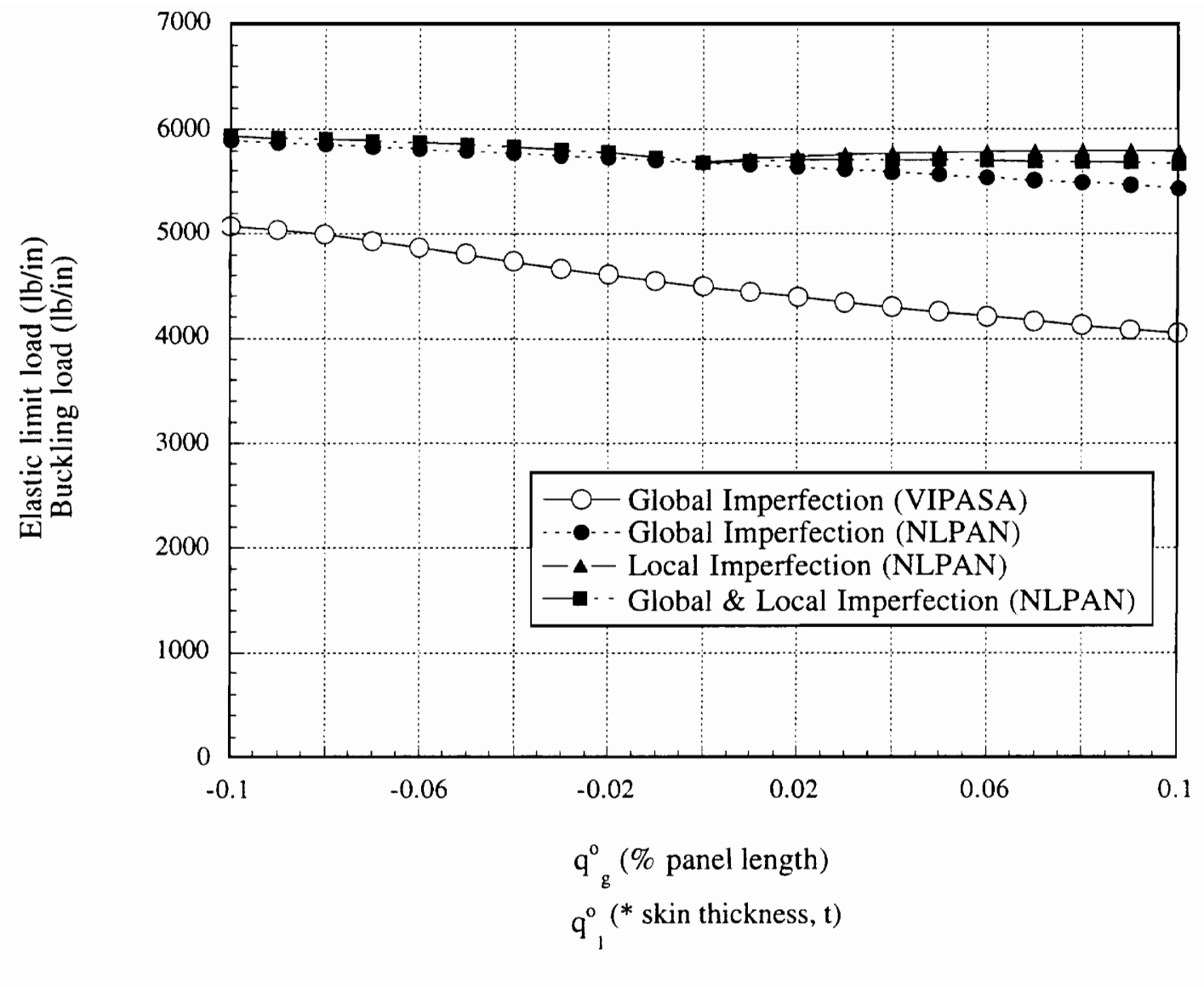

FIGURE 33. Imperfection sensitivity of Design 2 for NLPANOPT design with an initial global imperfection shape $($ Design load $=5000 \mathrm{lb} / \mathrm{in})$. 
fections is about $400 \mathrm{lb} / \mathrm{in}$ in both cases. The difference in postbuckling behavior is shown by the VIPASA results where Design 1 buckles between 600-1000 lb/in, except for a negative bow of 0.0481 in., and Design 2 buckles between 4000-5200 lb/in. The increase in buckling load for Design 1 at a negative bowing imperfection of $0.0481 \mathrm{in}$. is due to a switch from a local buckling load to a global buckling load.

Table 8: Summary of NLPANOPT designs with initial global imperfection shape for

\begin{tabular}{|c|c|c|}
\hline & Design 1 & Design 2 \\
\hline \hline $\mathrm{b}$ (in.) & 2.74207 & 2.88014 \\
\hline $\mathrm{t}_{1}\left(0^{\circ}{ }_{\text {skin }}\right)$ & 1 & 4 \\
\hline $\mathrm{t}_{2}\left(90^{\circ}{ }_{\text {skin }}\right)$ & 1 & 1 \\
\hline $\mathrm{t}_{3}\left( \pm 45^{\circ}{ }_{\text {skin }}\right)$ & 1 & 3 \\
\hline $\mathrm{t}_{4}\left(0^{\circ}{ }_{\text {blade }}\right)$ & 13 & 6 \\
\hline $\mathrm{t}_{5}\left(90^{\circ}{ }_{\text {blade }}\right)$ & 1 & 2 \\
\hline $\mathrm{t}_{6}\left( \pm 45^{\circ}{ }_{\text {blade }}\right)$ & 3 & 5 \\
\hline \begin{tabular}{c} 
Unit Weight $_{(\text {lbs. })}$ \\
\hline$\lambda_{\text {global }}$
\end{tabular} & 2.6312 & 3.9796 \\
\hline$\lambda_{\text {local }}$ & $0.817(1.23)$ & 1.47 \\
\hline $\mathrm{m}_{\text {local }}$ & 12 & 0.899 \\
\hline
\end{tabular}

Overall, for the axial compressive load of $5000 \mathrm{lb} / \mathrm{in}$, the nonlinear analysis with the inclusion of initial imperfections allows designs to be found that are lower in weight and less imperfection sensitive than designs found with a linear analysis. 


\subsubsection{Case 4: $10000 \mathrm{lb} /$ in Design Load}

The rounded-off design results for the $10000 \mathrm{lb} / \mathrm{in}$ axial compressive load are listed in Table 9. The continuous design results are listed in parentheses for the NLPANOPT design with an initial global imperfection shape.

Table 9: Summary of panel designs for $\mathbf{N}_{x}=10000 \mathrm{lb} / \mathrm{in}$.

\begin{tabular}{|c|c|c|c|c|}
\hline & PASCO & $\begin{array}{c}\text { PASCO } w / \\
\text { imperfection }\end{array}$ & NLPANOPT & $\begin{array}{c}\text { NLPANOPT } \\
\text { w/ } \\
\text { imperfection }\end{array}$ \\
\hline \hline $\mathrm{b}$ (in.) & 2.37195 & 2.92334 & 2.39695 & 3.43458 \\
\hline $\mathrm{t}_{1}\left(0^{\circ}{ }_{\text {skin }}\right)$ & 8 & 1 & 6 & $3(1.97)$ \\
\hline $\mathrm{t}_{2}\left(90^{\circ}{ }_{\text {skin }}\right)$ & 2 & 1 & 1 & $2(1.25)$ \\
\hline $\mathrm{t}_{3}\left( \pm 45^{\circ}{ }_{\text {skin }}\right)$ & 2 & 5 & 3 & $1(1.01)$ \\
\hline $\mathrm{t}_{4}\left(0^{\circ}{ }_{\text {blade }}\right)$ & 23 & 21 & 26 & $22(19.66)$ \\
\hline $\mathrm{t}_{5}\left(90^{\circ}{ }_{\text {blade }}\right)$ & 1 & 1 & 1 & $1(1)$ \\
\hline $\mathrm{t}_{6}\left( \pm 45^{\circ}{ }_{\text {blade }}\right)$ & 2 & 4 & 1 & $2(1.12)$ \\
\hline $\begin{array}{c}\left.\mathrm{Unit}_{\text {nit }} \text { (lbs. }\right) \\
(\text { lbht }\end{array}$ & 5.0791 & 5.3660 & 4.9743 & 4.4980 \\
\hline$\lambda_{\text {global }}$ & 1.01 & 1.37 & 1.05 & $0.851(1.91)$ \\
\hline$\lambda_{\text {local }}$ & 1.04 & 1.05 & 1.06 & 0.322 \\
\hline $\mathrm{m}_{\text {local }}$ & 9 & 11 & 5 & 13 \\
\hline
\end{tabular}

For the rounded-off designs with no imperfections, the NLPANOPT design weighs about $2 \%$ less than the PASCO design. The continuous PASCO design is buckling load critical and the continuous NLPANOPT design is limit load critical. The load versus end shortening behavior for these two designs is shown in Figure 34. The PASCO design is slightly stiffer than the NLPANOPT design. The imperfection sensitivity is shown in Figure 35 for the PASCO design and Figure 36 for the NLPANOPT design. Both designs display sensi- 


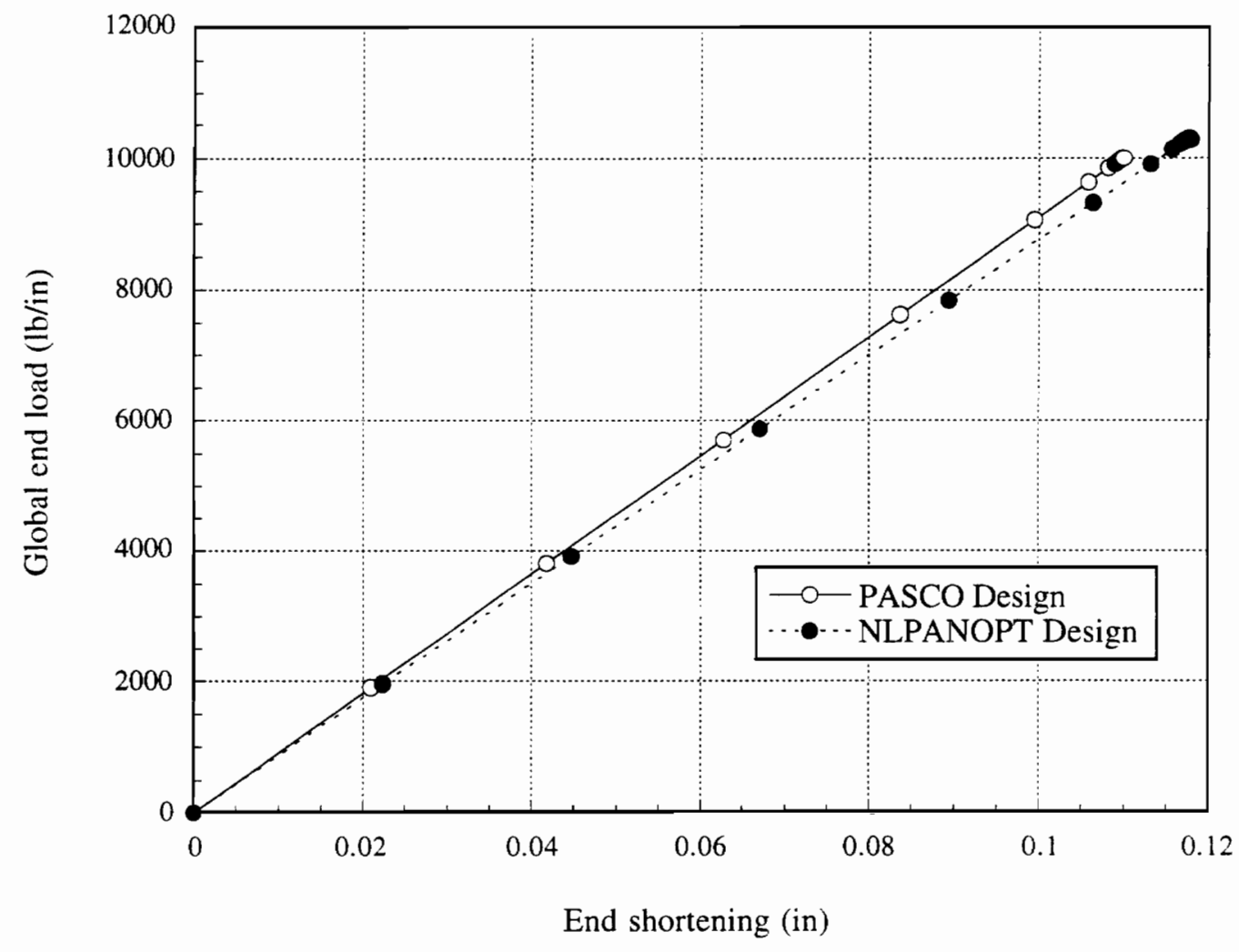

FIGURE 34. Load vs. end shortening for PASCO and NLPANOPT designs with no initial imperfections (Design load $=10000 \mathrm{lb} / \mathrm{in}$ ). 


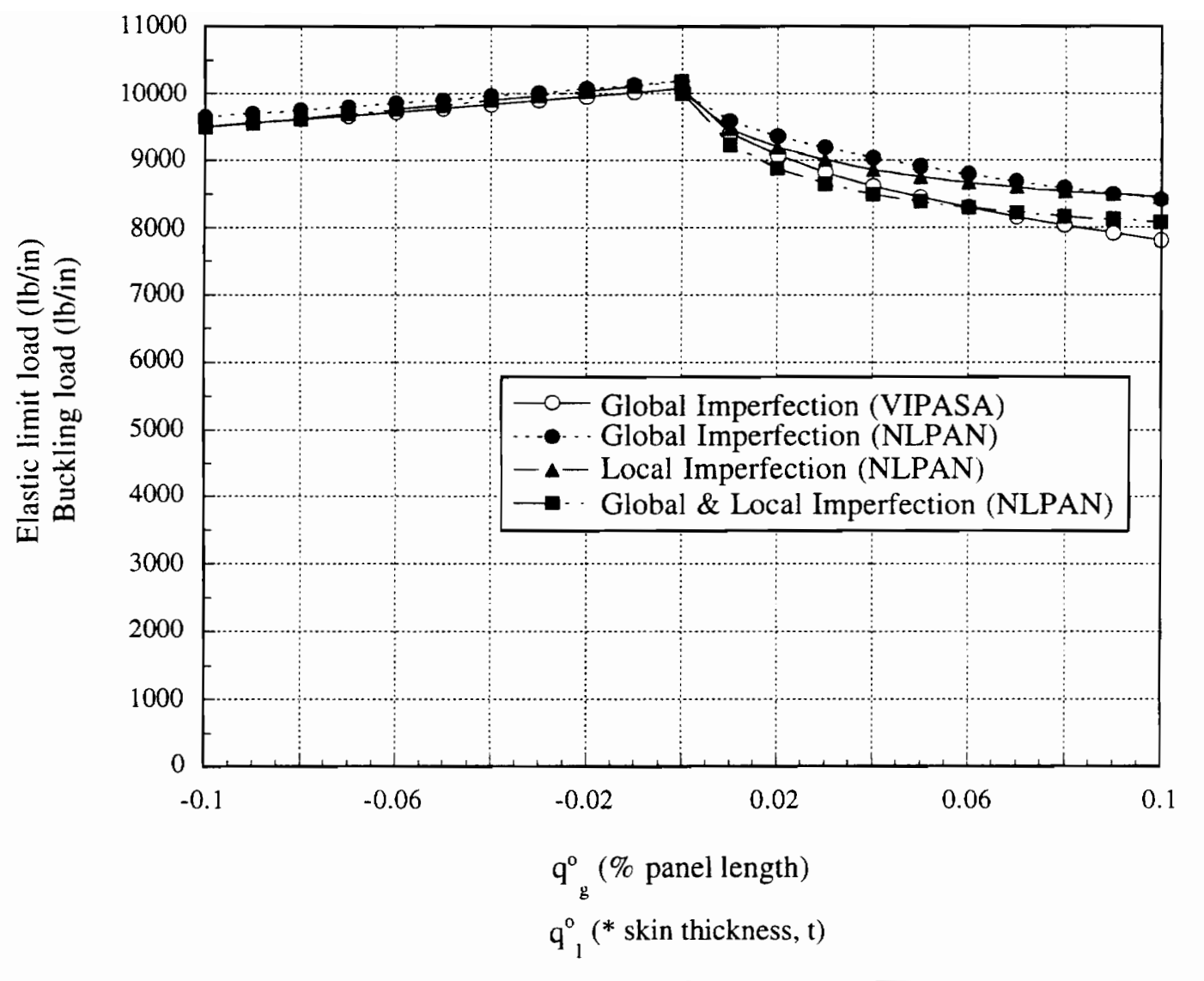

FIGURE 35. Imperfection sensitivity of PASCO design with no initial imperfections (Design load $=10000 \mathrm{lb} / \mathrm{in}$ ). 


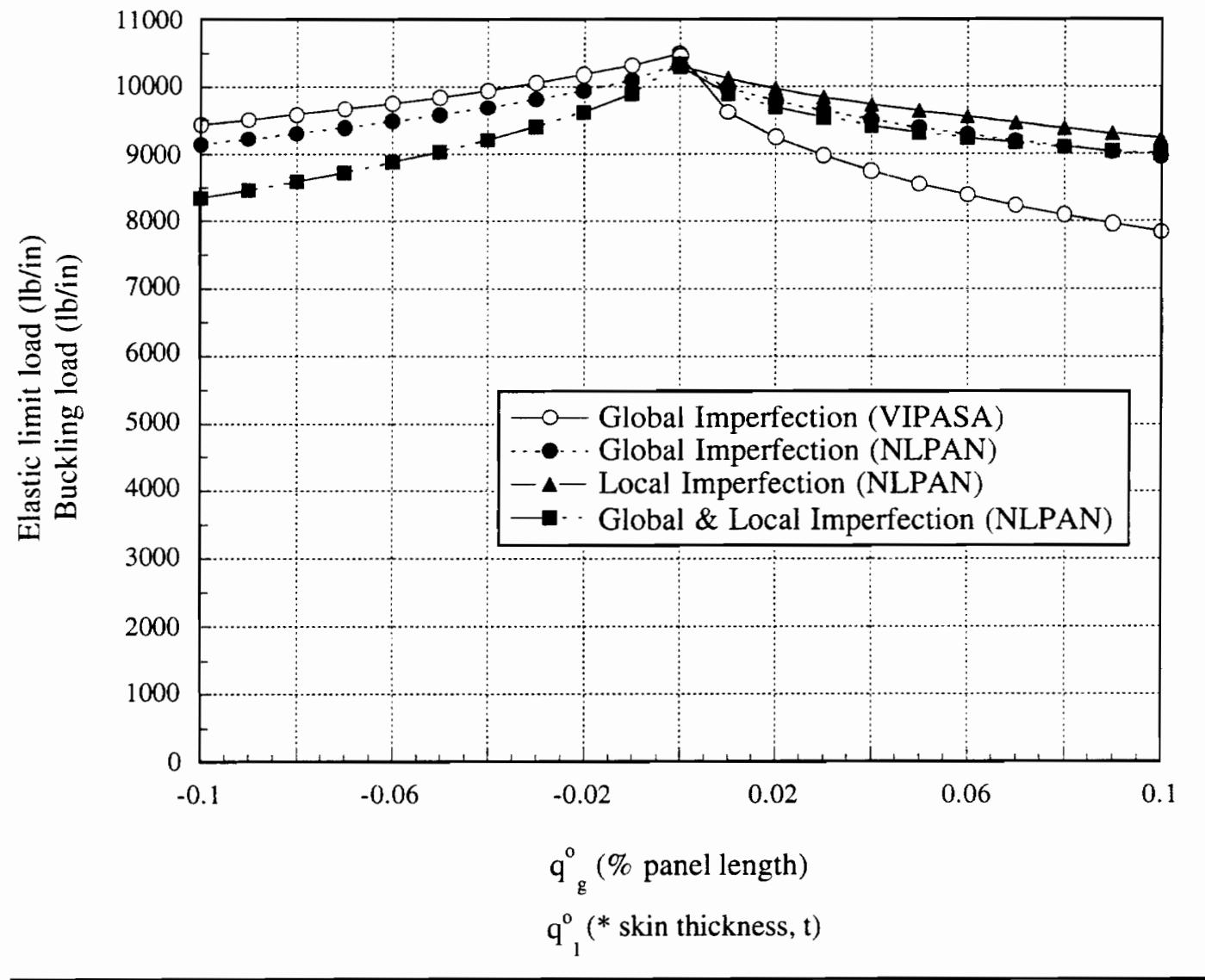

FIGURE 36. Imperfection sensitivity of NLPANOPT design with no initial imperfections (Design load $=10000 \mathrm{lb} / \mathrm{in}$ ). 
tivity behavior seen previously for designs that have nearly simultaneous buckling modes. The postbuckling strength is not taken advantage of for this NLPANOPT design.

With the addition of initial global imperfection shapes, the rounded-off PASCO design increases in weight by $5.6 \%$, but the rounded-off NLPANOPT design decreases in weight by $9.6 \%$. The imperfection sensitivity of these designs obtained by including initial imperfections is shown in Figure 37 for the PASCO design and Figure 38 for the NLPANOPT design. The PASCO design buckles above the design load for all bowing imperfections. NLPAN predicts the panel to carry the required load for only positive global imperfections or small local, negative global, and combined imperfections.

The rounded-off NLPANOPT design (see Figure 38 ) analyzed by NLPAN remains well above the design load for all imperfection amplitudes. Note that the panel is insensitive to local imperfection amplitudes, a result seen for previous locally postbuckled designs. Considering the amount that the design remains above the design load, the continuous design (shown in parentheses in Table 9) was also analyzed for its imperfection sensitivity to global imperfection amplitudes. The result is shown in Figure 39. The rounded-off design is $23 \%$ heavier compared to the continuous design. This result shows a disadvantage of needing to round-off continuous design variables.

For the axial compressive load of $10000 \mathrm{lb} / \mathrm{in}$, the nonlinear analysis with the inclusion of initial imperfections allows a design to be found that is lower in weight and less imperfection sensitive than a design found with a linear analysis.

\subsection{Effect of Skin Laminate Stacking Sequence}

As discussed at the end of Section 4.1.2, since the stacking sequence is prescribed in the current design procedure, the resulting designs may not be truly optimal. By allowing the 


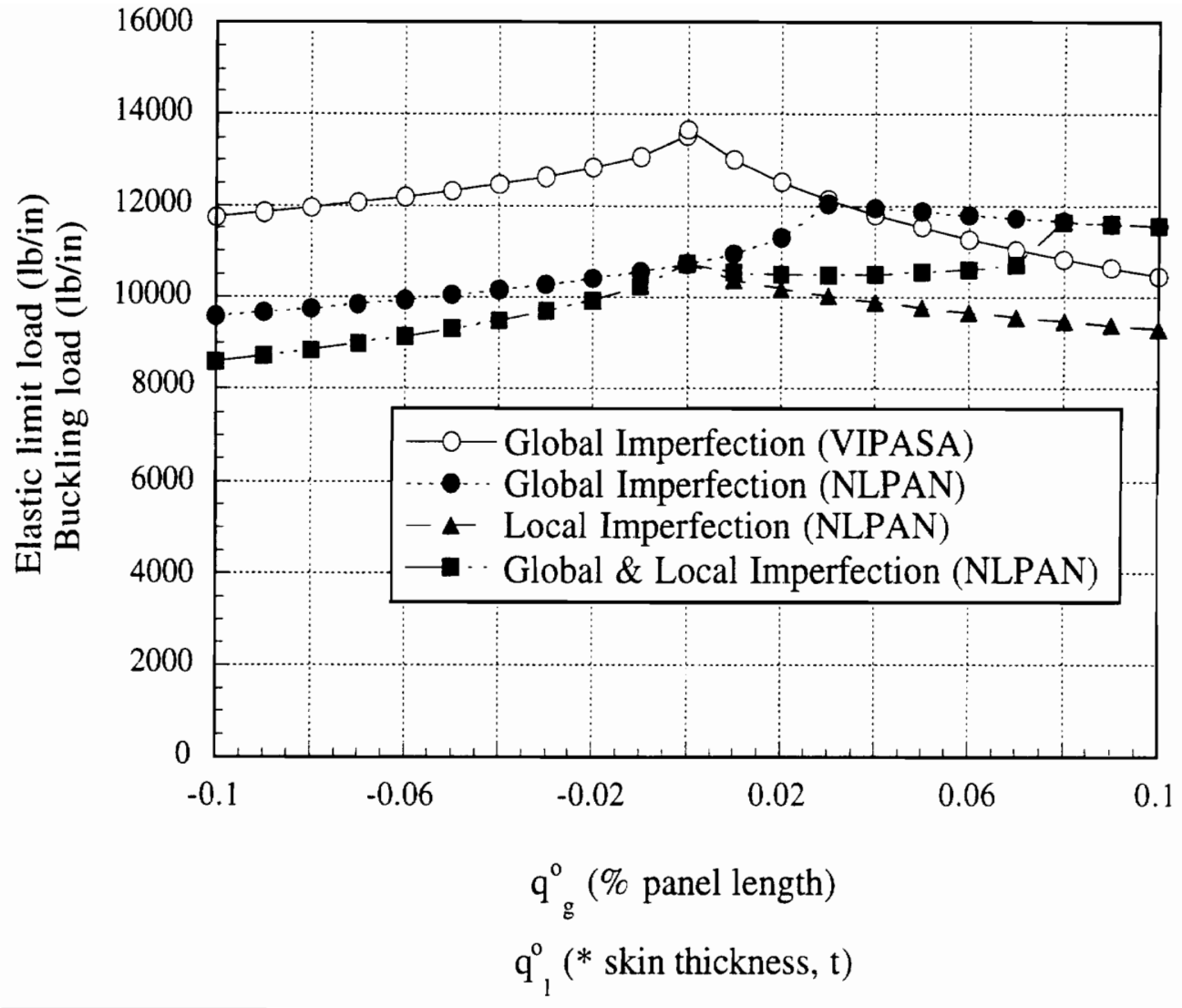

FIGURE 37. Imperfection sensitivity of PASCO design with initial bowing imperfection $($ Design load $=10000 \mathrm{lb} / \mathrm{in})$. 


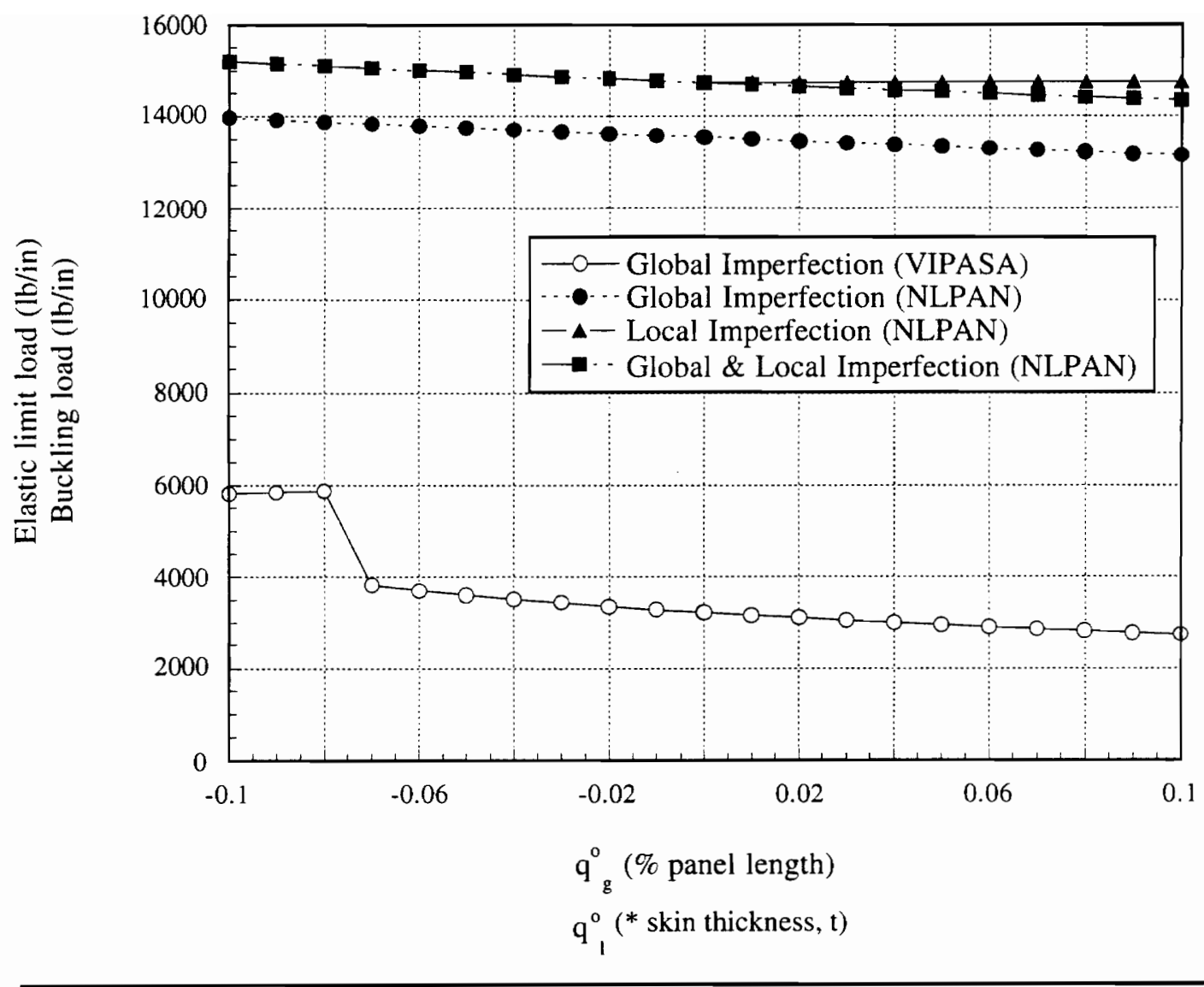

FIGURE 38. Imperfection sensitivity of NLPANOPT design with initial global imperfection shape (Design load $=10000 \mathrm{lb} / \mathrm{in}$ ). 


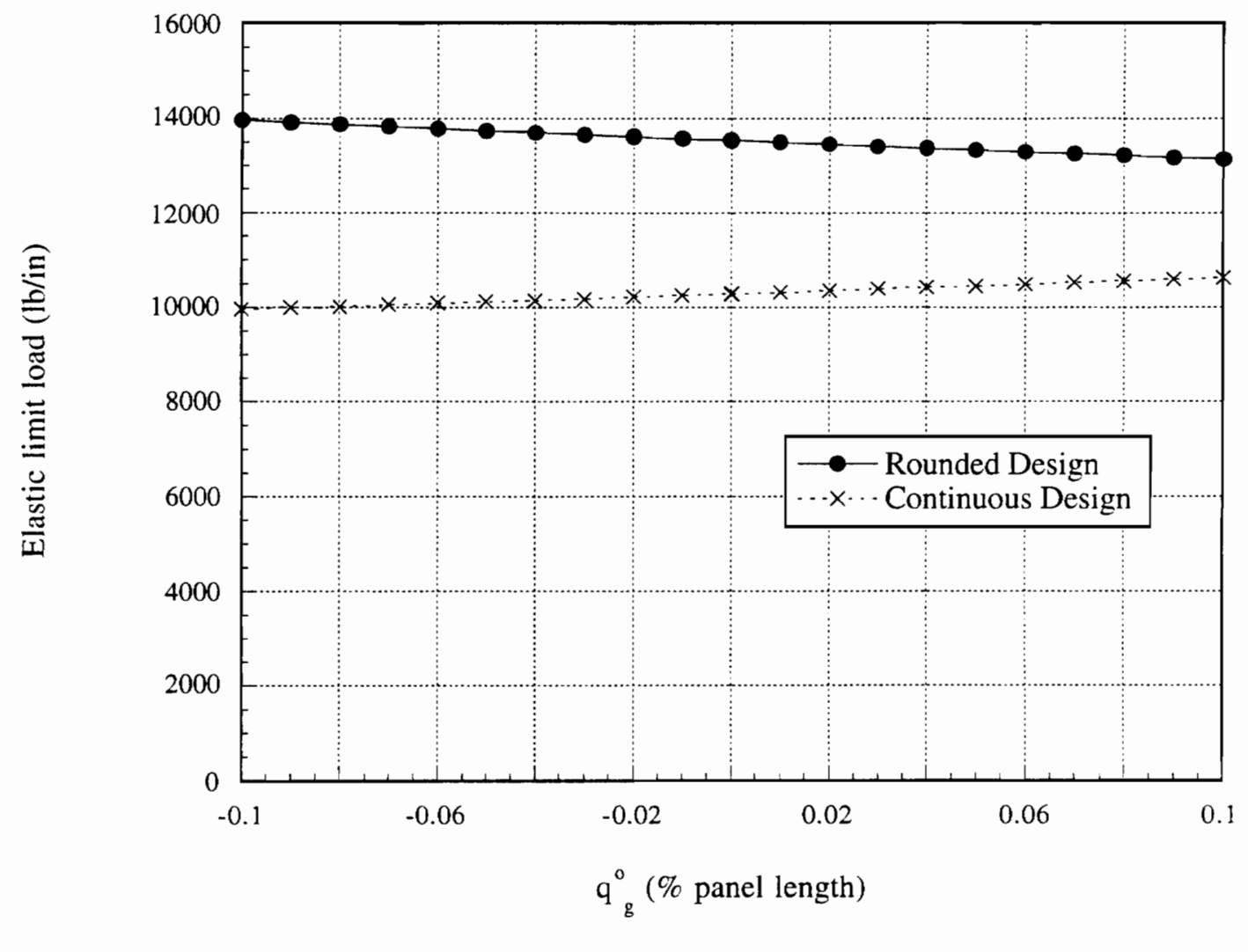

FIGURE 39. Comparison of imperfection sensitivity between the continuous and rounded-off NLPANOPT designs with initial global imperfection shape using NLPAN (Design load $=10000 \mathrm{lb} / \mathrm{in}$ ). 
stacking sequence to be variable, a lower weight design may be possible. To investigate the possibility of achieving a lower weight design with the current design procedure, the skin laminate stacking sequence was manually changed for the blade-stiffened panel designed with an initial global imperfection under a compressive load of $1000 \mathrm{lb} / \mathrm{in}$. First, the rounded design shown in column 5 of Table 5 was reanalyzed with 5 different skin laminate stacking sequences, $\left[ \pm 45_{1} / 0_{1} / 90_{1}\right]_{S},\left[0_{1} / \pm 45_{1} / 90_{1}\right]_{S},\left[0_{1} / 90_{1} / \pm 45_{1}\right]_{S}$, $\left[90_{1} / 0_{1} / \pm 45_{1}\right]_{S}$, and $\left[90_{1} / \pm 45_{1} / 0_{1}\right]_{S}$, to descry if the final design could be improved, resulting in an elastic limit load greater than the design load, by changing only the skin laminate stacking sequence. The predicted elastic limit loads for a positive and a negative global imperfection of 0.0481 in. (0.1\% of the panel length) are shown in Table 10 (critical limit load values in bold lettering). The original stacking sequence, Skin 1, is listed in the first row. For each stacking sequence, material failure does not occur before the elastic limit load is reached. No alternative stacking sequence results in a higher maximum load, thus the original stacking sequence, $\left[ \pm 45_{1} / 90_{1} / 0_{1}\right]_{S}$, remains the optimal one for these dimensions.

Even though a higher maximum load was not found when the skin laminate stacking sequence was changed, a lower weight design may be possible with a different skin laminate stacking sequence if the dimensions are also changed. Therefore, the blade-stiffened panel was redesigned with each skin laminate stacking sequence for an axial compressive load of $1000 \mathrm{lb} / \mathrm{in}$ and an initial global imperfection amplitude of $0.0481 \mathrm{in}$. The final rounded-off designs are listed in Table 11 with the design from Section 4.1.2 listed in column 2.

A lower weight design compared to Skin 1 is obtained for Skin 3, $\left[0_{1} / \pm 45_{1} / 90_{1}\right]_{S}$. The weight is reduced by $3 \%$ compared to Skin 1 . The remaining skin laminate stacking 
sequences result in heavier designs. The load versus end shortening response for each skin laminate stacking sequence is shown in Figure 40. The skin laminate stacking sequences are listed in order of weight in the legend. Skin 1, Skin 2, Skin 4, Skin 5 and Skin 6 are $3.4 \%, 6.5 \%, 6.7 \%, 78 \%$ and $6.9 \%$ heavier than Skin 3, respectively. Each design buckles locally well below the design load, between $173 \mathrm{lb} /$ in and $293 \mathrm{lb} /$ in, except Skin 5 which buckles locally at $1180 \mathrm{lb} / \mathrm{in}$. It appears that the Skin 5 design is significantly heavier than the other designs because it does not use its local postbuckling behavior before the design load. The stiffest designs have the $\pm 45^{\circ}$ plies at the innermost layers, while the least stiff designs have the $90^{\circ}$ plies at the innermost layers. These least stiff designs also display dramatic drops in load carrying ability after the limit load unlike the remaining designs which display gradual drops in load carrying capability after the limit load.

Table 10: Effect of skin stacking sequence on panel response of $[ \pm 45 / 90 / 0]$ skin laminate design.

\begin{tabular}{|c|c|c|}
\hline \multirow{2}{*}{} & \multicolumn{2}{|c|}{ Elastic limit load (lb/in) } \\
\cline { 2 - 3 } & $+q_{g}^{o}$ & $-q_{g}^{o}$ \\
\hline $\begin{array}{c}\text { Skin } 1 \\
{\left[ \pm 45_{1} / 90_{1} / 0_{1}\right]_{s}}\end{array}$ & 1122 & $\mathbf{1 0 1 5}$ \\
\hline $\begin{array}{c}\text { Skin } 2 \\
{\left[ \pm 45_{1} / 0_{1} / 90_{1}\right]_{s}}\end{array}$ & 1103 & $\mathbf{8 1 7}$ \\
\hline $\begin{array}{c}\text { Skin } 3 \\
{\left[0_{1} / \pm 45_{1} / 90_{1}\right]_{S}}\end{array}$ & 845 & $\mathbf{7 5 0}$ \\
\hline $\begin{array}{c}\text { Skin } 4 \\
{\left[0_{1} / 90_{1} / \pm 45_{1}\right]_{S}}\end{array}$ & 1063 & $\mathbf{7 3 6}$ \\
\hline $\begin{array}{c}\text { Skin } 5 \\
{\left[90_{1} / 0_{1} / \pm 45_{1}\right]_{S}}\end{array}$ & $\mathbf{9 2 6}$ & 992 \\
\hline $\begin{array}{c}\text { Skin } 6 \\
{\left[90_{1} / \pm 45_{1} / 0_{1}\right]_{S}}\end{array}$ & & \\
\hline
\end{tabular}




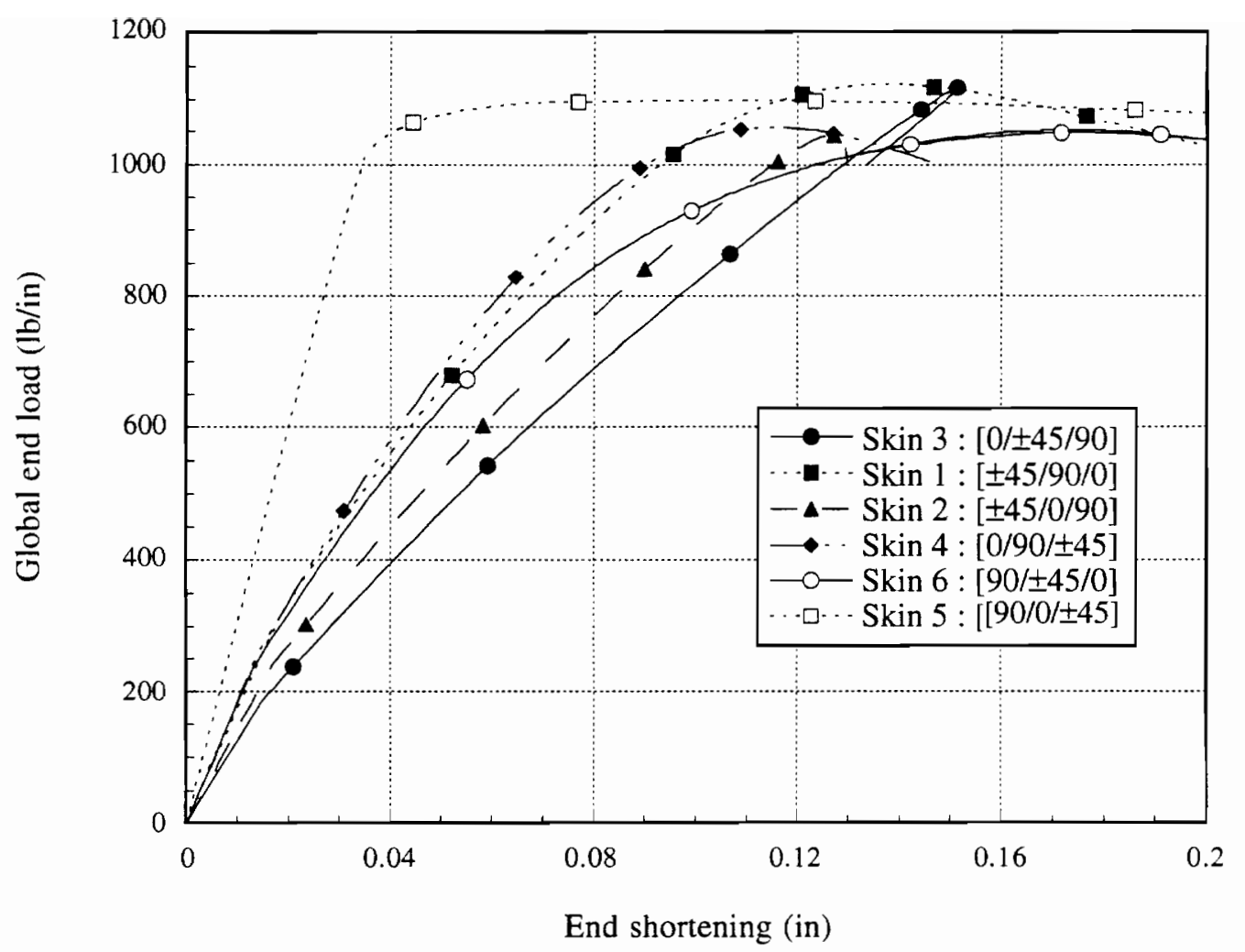

FIGURE 40. Load versus end shortening of rounded-off designs for bladestiffened panel with different skin laminate stacking sequences (Design load $=1000 \mathrm{lb} / \mathrm{in}$ ). 
Table 11: Summary of NLPANOPT designs for different skin laminate stacking sequences (Design Load $=1000 \mathrm{lb} / \mathrm{in}$ ).

\begin{tabular}{|c|c|c|c|}
\hline & $\begin{array}{c}\text { Skin } 1 \\
{\left[ \pm 45_{1} / 90_{1} / 0_{1}\right]_{S}}\end{array}$ & $\begin{array}{c}\text { Skin } 2 \\
{\left[ \pm 45_{1} / 0_{1} / 90_{1}\right]_{S}}\end{array}$ & $\begin{array}{c}\text { Skin 3 } \\
{\left[0_{1} / \pm 45_{1} / 90_{1}\right]_{S}}\end{array}$ \\
\hline \hline $\mathrm{b}$ (in.) & 1.79196 & 1.92817 & 1.84473 \\
\hline $\mathrm{t}_{1}\left(0^{\circ}{ }_{\text {skin }}\right)$ & 1 & 1 & 1 \\
\hline $\mathrm{t}_{2}\left(90^{\circ}{ }_{\text {skin }}\right)$ & 1 & 1 & 1 \\
\hline $\mathrm{t}_{3}\left( \pm 45^{\circ}{ }_{\text {skin }}\right)$ & 1 & 1 & 2 \\
\hline $\mathrm{t}_{4}\left(0_{\text {blade }}^{\circ}\right)$ & 6 & 3 & 2 \\
\hline $\mathrm{t}_{5}\left(90^{\circ}{ }_{\text {blade }}\right)$ & 1 & 2 & 2 \\
\hline $\mathrm{t}_{6}\left( \pm 45^{\circ}\right.$ blade $)$ & 1 & 1.3978 & 1.3124 \\
\hline $\begin{array}{c}\text { Unit Weight } \\
(\text { lbs. })\end{array}$ & 1.3575 & 1.02 & 0.719 \\
\hline$\lambda_{\text {global }}$ & 1.17 & 0.248 & 0.173 \\
\hline$\lambda_{\text {local }}$ & 0.293 & 10 & 8 \\
\hline $\mathrm{m}_{\text {local }}$ & 11 & 2 & 2 \\
\hline
\end{tabular}

As was done for Skin 1, the design of Skin 3 is reanalyzed it with the other skin laminate stacking sequences to determine if a higher maximum load is possible. The predicted elastic limit loads for a positive and a negative global imperfection of 0.0481 in. ( $0.1 \%$ of the panel length) are shown in Table12 (critical limit load values in bold lettering). For each stacking sequence, material failure does not occur before the elastic limit load is reached. No alternative stacking sequence results in a higher maximum load, thus the original stacking sequence, $\left[0_{1} / \pm 45_{1} / 90_{1}\right]_{S}$, remains the optimal one for these dimensions.

Since the current design procedure requires the stacking sequence to be prescribed, lower weight designs, which are possible by changing the stacking sequences, are not detected. By employing an optimizer with the ability to determine the discrete number of plies and sequence of orientations, a global optimal design would be discovered. As discussed at the 
end of Section 4.1.2, one possibility is the use of a genetic algorithm that contains this ability [46]. The genetic algorithm, which handles discrete variables, would also eliminate the need for rounding-off the designs.

Table 11: (Con't) Summary of NLPANOPT designs for different skin laminate stacking sequences (Design Load $=1000 \mathrm{lb} / \mathrm{in}$ ).

\begin{tabular}{|c|c|c|c|}
\hline & $\begin{array}{c}\text { Skin } 4 \\
{\left[0_{1} / 90_{1} / \pm 45_{1}\right]_{S}}\end{array}$ & $\begin{array}{c}\text { Skin } 5 \\
{\left[90_{1} / 0_{1} / \pm 45_{1}\right]_{S}}\end{array}$ & $\begin{array}{c}\text { Skin } 6 \\
{\left[90_{1} / \pm 45_{1} / 0_{1}\right]_{S}}\end{array}$ \\
\hline \hline $\mathrm{b}$ (in.) & 1.74401 & 1.61679 & 1.94597 \\
\hline $\mathrm{t}_{1}\left(0^{\circ}{ }_{\text {skin }}\right)$ & 1 & 3 & 1 \\
\hline $\mathrm{t}_{2}\left(90^{\circ}{ }_{\text {skin }}\right)$ & 1 & 1 & 1 \\
\hline $\mathrm{t}_{3}\left( \pm 45^{\circ}{ }_{\text {skin }}\right)$ & 1 & 2 & 6 \\
\hline $\mathrm{t}_{4}\left(0_{\text {blade }}^{\circ}\right)$ & 7 & 7 & 1 \\
\hline $\mathrm{t}_{5}\left(90^{\circ}\right.$ blade $)$ & 1 & 2 & 1 \\
\hline $\mathrm{t}_{6}\left( \pm 45^{\circ}\right.$ blade $)$ & 1 & 2.3453 & 1.4031 \\
\hline $\begin{array}{c}\text { Unit Weight } \\
(\text { lbs. })\end{array}$ & 1.4007 & 1.37 & 1.48 \\
\hline$\lambda_{\text {global }}$ & 1.20 & 1.18 & 0.246 \\
\hline$\lambda_{\text {local }}$ & 0.266 & 8 & 15 \\
\hline $\mathrm{m}_{\text {local }}$ & 9 & 2 & 1 \\
\hline
\end{tabular}

\subsection{Design of Blade-Stiffened Panel with Flanges}

The second configuration studied is similar to the first (see Figure 7), but with added stiffener flanges. An eight T-stiffened graphite-epoxy panel subject to an axial compressive loading is modelled using a stiffener-unit representation with symmetry conditions listed in equation (34) applied at the longitudinal edges $(Y=0, B)$ of the unit cell. The geometry and design variables of the stiffener-unit representation are shown in Figure 41. Designs are obtained for an axial compressive load of $\mathrm{N}_{\mathrm{x}}=1000 \mathrm{lb} / \mathrm{in}$. As in Section 4.1, the panel 


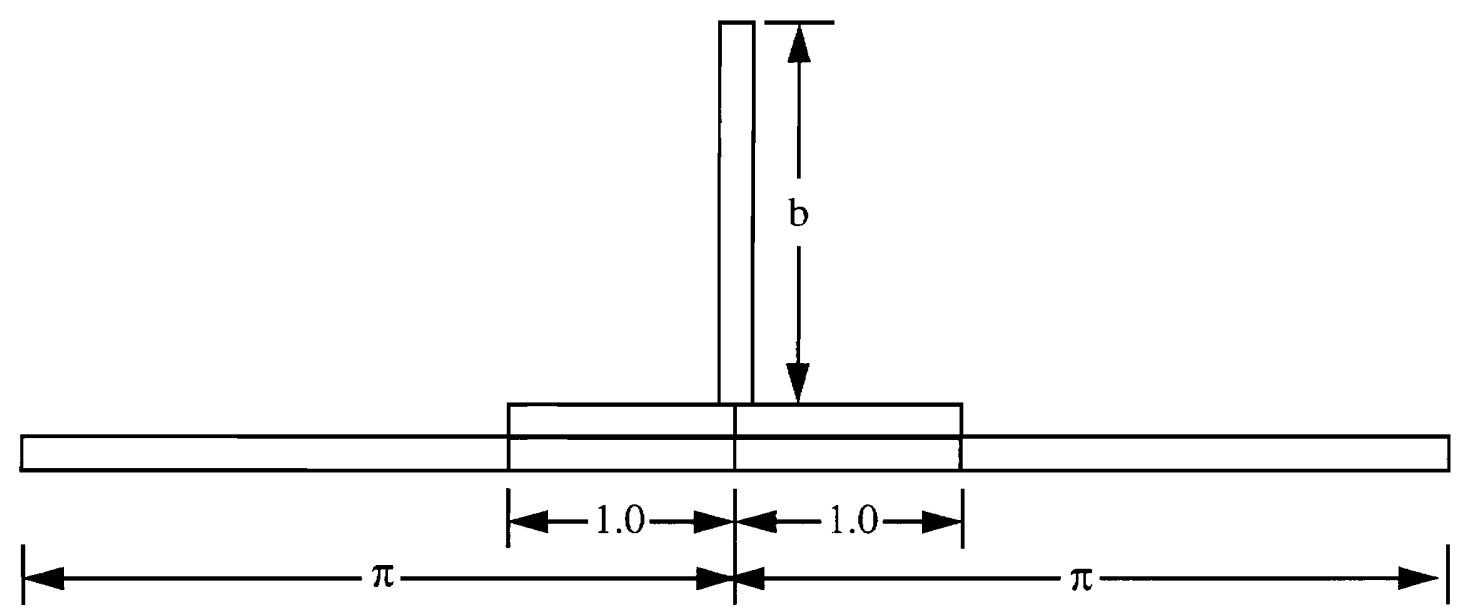

(a) Stiffener-unit representation (all dimensions are in inches).

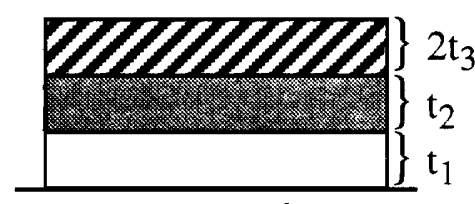

symmetry plane SKIN

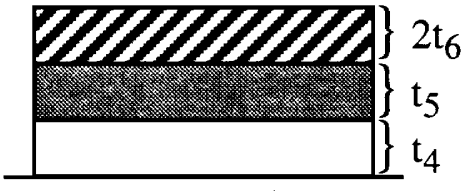

symmetry plane FLANGE

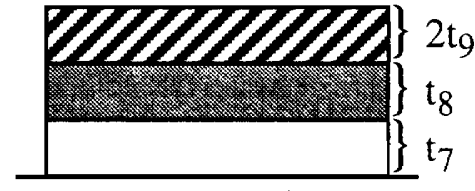

symmetry plane BLADE

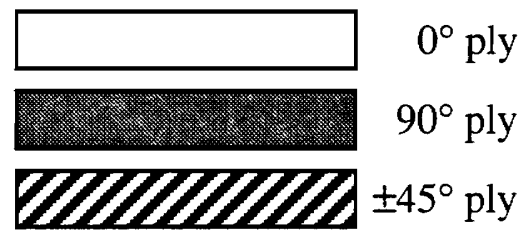

(b) Design Variable Assignments.

Figure 41. T-stiffened panel geometry and possible design variables. 
is designed using: 1) PASCO with no imperfections, 2) PASCO with an initial bowing imperfection of 0.0481 in. ( $0.1 \%$ of the panel length), 3) NLPANOPT with no imperfections, and 4) NLPANOPT with an initial imperfection in the shape of the global mode and an amplitude of 0.0481 in. ( $0.1 \%$ of the panel length).

Table 12: Effect of skin stacking sequence on panel response of $[0 / \pm 45 / 90]$ skin laminate design.

\begin{tabular}{|c|c|c|}
\hline \multirow{2}{*}{} & \multicolumn{2}{|c|}{ Elastic limit load (lb/in) } \\
\cline { 2 - 3 } & $+q_{g}^{o}$ & $-q_{g}^{o}$ \\
\hline $\begin{array}{c}\text { Skin } 1 \\
{\left[ \pm 45_{1} / 90_{1} / 0_{1}\right]_{s}}\end{array}$ & $\mathbf{6 6 1}$ & 728 \\
\hline $\begin{array}{c}\text { Skin } 2 \\
{\left[ \pm 45_{1} / 0_{1} / 90_{1}\right]_{s}}\end{array}$ & $\mathbf{7 0 8}$ & 826 \\
\hline $\begin{array}{c}\text { Skin } 3 \\
{\left[0_{1} / \pm 45_{1} / 90_{1}\right]_{s}}\end{array}$ & 1174 & $\mathbf{1 1 1 7}$ \\
\hline $\begin{array}{c}\text { Skin } 4 \\
{\left[0_{1} / 90_{1} / \pm 45_{1}\right]_{s}}\end{array}$ & $\mathbf{6 8 5}$ & 774 \\
\hline $\begin{array}{c}\text { Skin } 5 \\
{\left[90_{1} / 0_{1} / \pm 45_{1}\right]_{s}}\end{array}$ & $\mathbf{5 5 1}$ & 598 \\
\hline $\begin{array}{c}\text { Skin } 6 \\
{\left[90_{1} / \pm 45_{1} / 0_{1}\right]_{s}}\end{array}$ & $\mathbf{5 0 5}$ & 550 \\
\hline
\end{tabular}

\subsection{1 $1000 \mathrm{lb} / \mathrm{in}$ Design Load}

The design results for the $1000 \mathrm{lb} /$ in design load are listed in Table 13 with the continuous results listed in parentheses. Comparing the rounded-off results for the panels designed with no initial imperfections, the NLPANOPT design weighs $28 \%$ less than the PASCO design. The NLPANOPT design has a minimum skin and flange thickness, but an increase in the $0^{\circ}$ ply in the blade by one ply thickness and an increase in the blade height by $1 \%$ compared to the PASCO design. The continuous NLPANOPT design is limit load critical 
and buckles at $46 \%$ of the design load. The continuous PASCO design, however, has simultaneous global and local buckling modes.

Table 13: Summary of panel designs with flanges for $N_{x}=1000 \mathrm{lb} / \mathrm{in}$.

\begin{tabular}{|c|c|c|c|c|}
\hline & PASCO & $\begin{array}{c}\text { PASCO w/ } \\
\text { imperfection }\end{array}$ & NLPANOPT & $\begin{array}{c}\text { NLPANOPT } \\
\text { w/ } \\
\text { imperfection }\end{array}$ \\
\hline \hline $\mathrm{b}$ (in.) & 1.70754 & 1.9370 & 1.72157 & 1.60160 \\
\hline $\mathrm{t}_{1}\left(0^{\circ}{ }_{\text {skin }}\right)$ & $1(1)$ & $1(1)$ & $1(1)$ & $1(1)$ \\
\hline $\mathrm{t}_{2}\left(90^{\circ}{ }_{\text {skin }}\right)$ & $1(1)$ & $1(1)$ & $1(1)$ & $1(1)$ \\
\hline $\mathrm{t}_{3}\left( \pm 45^{\circ}{ }_{\text {skin }}\right)$ & $2(1.33)$ & $2(1.43)$ & $1(1)$ & $1(1)$ \\
\hline $\mathrm{t}_{4}\left(0^{\circ}\right.$ flange $)$ & $5(4.54)$ & $4(4.40)$ & $1(1)$ & $1(1)$ \\
\hline $\mathrm{t}_{5}\left(90^{\circ}\right.$ flange $)$ & $1(1)$ & $1(1)$ & $1(1)$ & $1(1)$ \\
\hline $\mathrm{t}_{6}\left( \pm 45^{\circ}\right.$ flange $)$ & $1(1)$ & $1(1)$ & $1(1)$ & $1(1)$ \\
\hline $\mathrm{t}_{7}\left(0^{\circ}\right.$ blade $)$ & $4(3.41)$ & $4(4.13)$ & $5(4.40)$ & $7(6.82)$ \\
\hline $\mathrm{t}_{8}\left(90^{\circ}{ }_{\text {blade }}\right)$ & $1(1)$ & $1(1)$ & $1(1)$ & $1(1)$ \\
\hline $\mathrm{t}_{9}\left( \pm 45^{\circ}{ }_{\text {blade }}\right)$ & $1(1)$ & $1(1)$ & $1(1)$ & $1(1)$ \\
\hline $\mathrm{Unit}_{\text {Weight }}$ & 2.15997 & 2.14702 & 1.54320 & 1.61701 \\
$($ lbs. $)$ & $(1.81881)$ & $(1.94674)$ & $(1.50896)$ & $(1.60753)$ \\
\hline$\lambda_{\text {global }}$ & $1.17(1.0)$ & $1.59(1.59)$ & $1.14(1.05)$ & $1.18(1.16)$ \\
\hline$\lambda_{\text {local }}$ & $1.70(1.0)$ & $1.02(1.0)$ & $0.486(0.457)$ & $0.564(0.555)$ \\
\hline $\mathrm{m}_{\text {local }}$ & $10(12,13)$ & $13(12,13)$ & $12(11)$ & $13(13)$ \\
\hline & & & & \\
\hline
\end{tabular}

The imperfection sensitivity of the rounded-off PASCO design is shown in Figure 42. The rounded-off PASCO design buckles above the design load for all positive bowing imperfections and for negative bowing imperfection below $0.07 \%$ of the panel length. The nonlinear analysis also predicts the panel to carry the required load for these global imperfection amplitudes. With both global and local imperfection amplitudes, however, the panel carries the required load for negative imperfection amplitudes below $0.05 \%$ of 


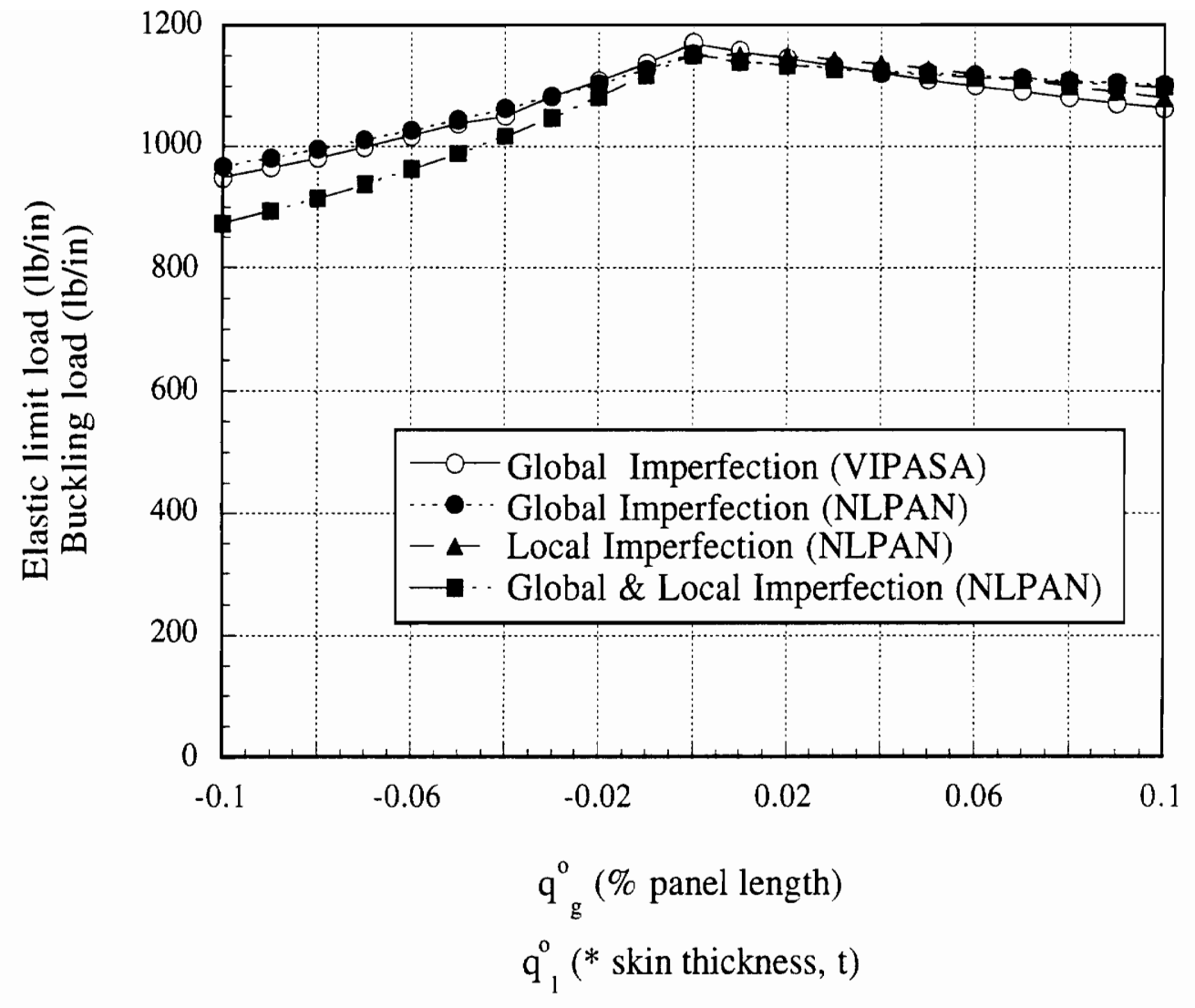

FIGURE 42. Imperfection sensitivity of the rounded-off PASCO design with no initial imperfections for panel with added flange (Design load $=1000 \mathrm{lb} / \mathrm{in}$ ). 
the panel length. Comparing this panel sensitivity with the continuous panel sensitivity, shown in Figure 43, the continuous design carries the design load only for the imperfection it was designed.

The imperfection sensitivity of the rounded-off NLPANOPT design is shown on Figure 44. The VIPASA analysis predicts the panel to buckle between $425 \mathrm{lb} / \mathrm{in}$ and $575 \mathrm{lb} / \mathrm{in}$. The nonlinear analysis predicts the panel to carry the design load for all positive global imperfections. Below a negative imperfection amplitude $0.03 \%$ of the panel length, the panel is unable to carry the design load. An increase in local imperfection amplitudes cause only a slight decrease in the maximum load carried. The addition of global and local imperfections cause the panel to be unable to carry the design load below $-0.02 \%$ of the panel length. The imperfection sensitivity of the continuous NLPANOPT design to global imperfection shapes is shown in Figure 45. Again, the continuous design is unable to carry the required load except for the imperfection it was designed.

Comparing the rounded-off designs obtained with an initial global imperfection (listed in column 3 and column 5 of Table 13, the NLPANOPT design weighs $25 \%$ less than the PASCO design. The NLPANOPT panel to buckles below the design load, at $564 \mathrm{lb} / \mathrm{in}$. The PASCO design increases the global eigenvalue 59\% above the design load. The imperfection sensitivity of the rounded-off PASCO design is shown in Figure 46. The panel buckles above the design load for all bowing imperfections. The nonlinear analysis predicts the panel to be capable of carrying the required load for a imperfections except for combined large negative global imperfections and local imperfections.

The imperfection sensitivity of the rounded-off NLPANOPT design is shown in Figure 47 . The nonlinear analysis predicts the panel to carry the required load for all imperfection amplitudes and shapes. The panel buckles below its maximum load for the entire range of 


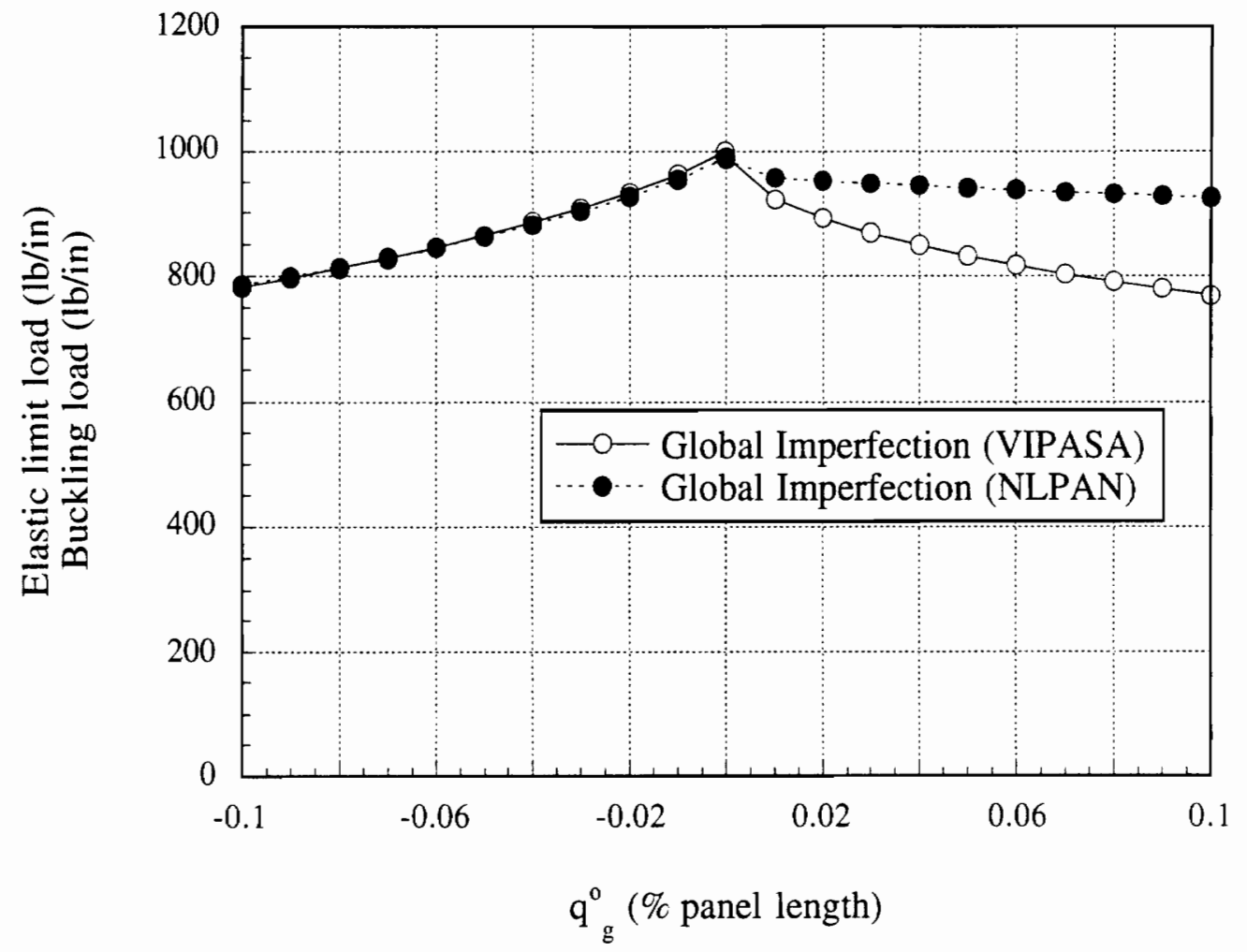

FIGURE 43. Imperfection sensitivity of the continuous PASCO flange design with no initial imperfections to global imperfection shapes $($ Design load $=1000$ lb/in). 


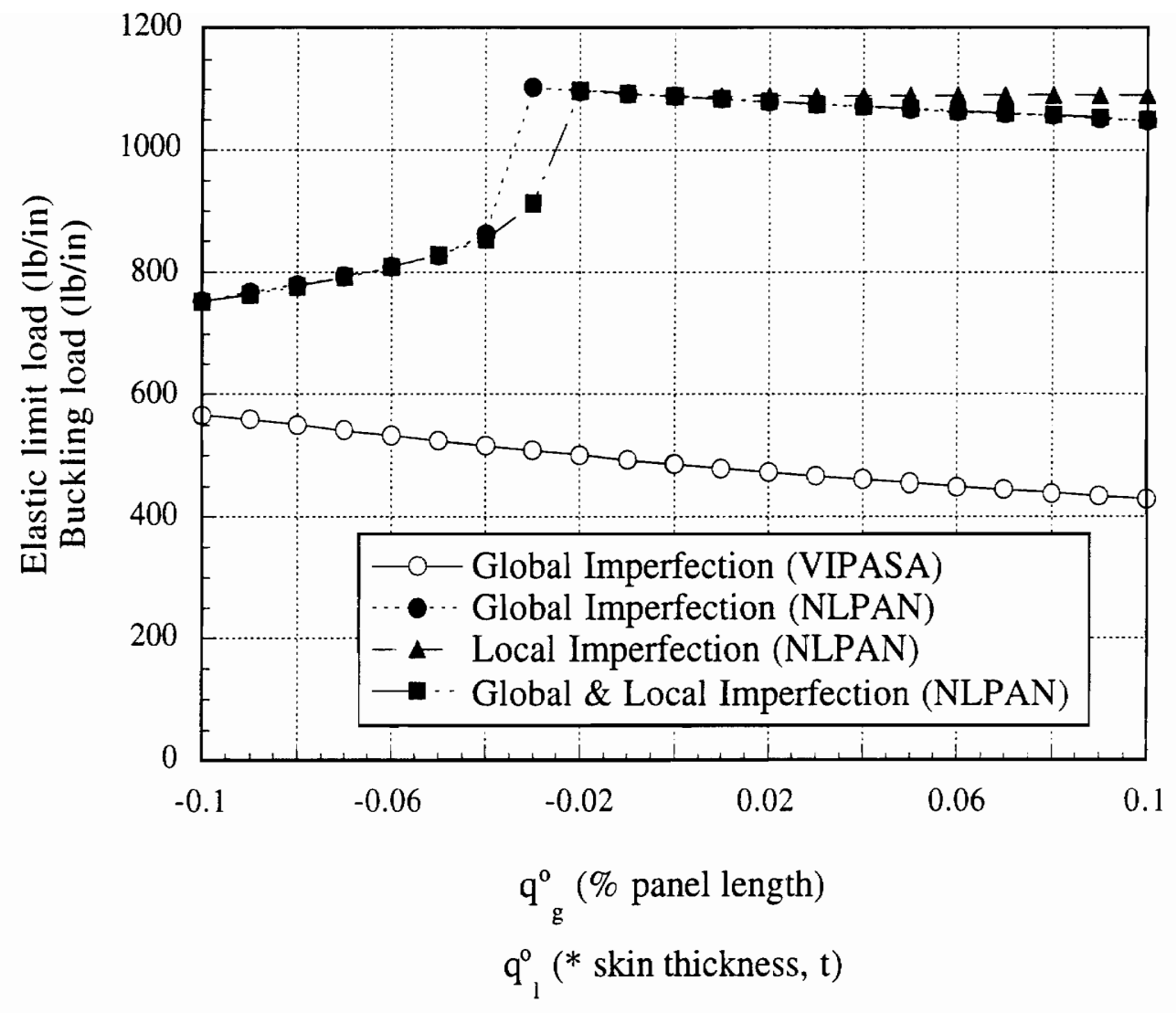

FIGURE 44. Imperfection sensitivity of the rounded-off NLPANOPT flange design with no initial imperfections (Design load $=1000 \mathrm{lb} / \mathrm{in})$. 


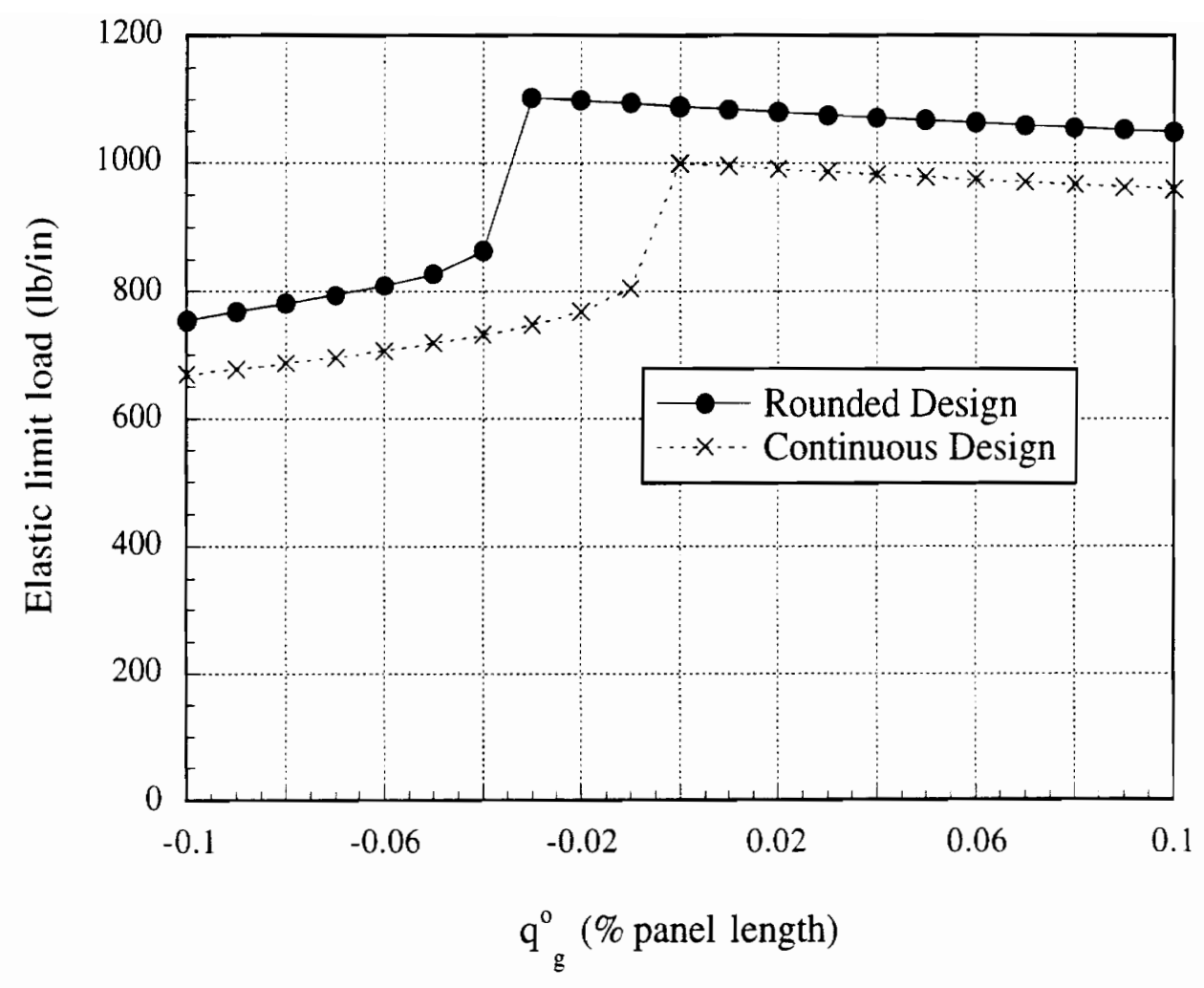

FIGURE 45. Imperfection sensitivity of the continuous NLPANOPT flange design with no initial imperfections to global imperfection shapes (Design load $=1000 \mathrm{lb} / \mathrm{in}$ ). 


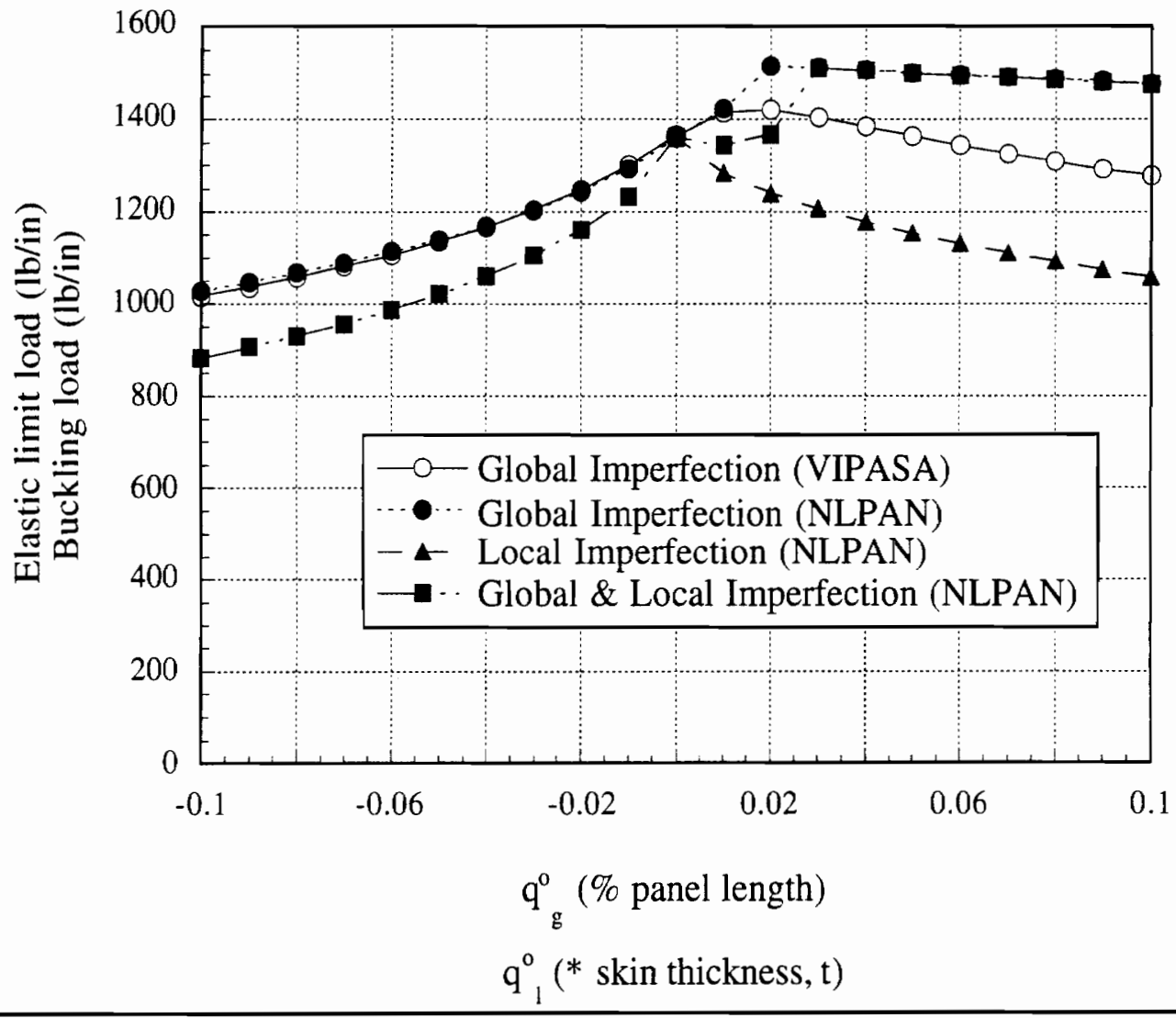

FIGURE 46. Imperfection sensitivity of the rounded-off PASCO flange design with bowing imperfection (Design load $=1000 \mathrm{lb} / \mathrm{in})$. 


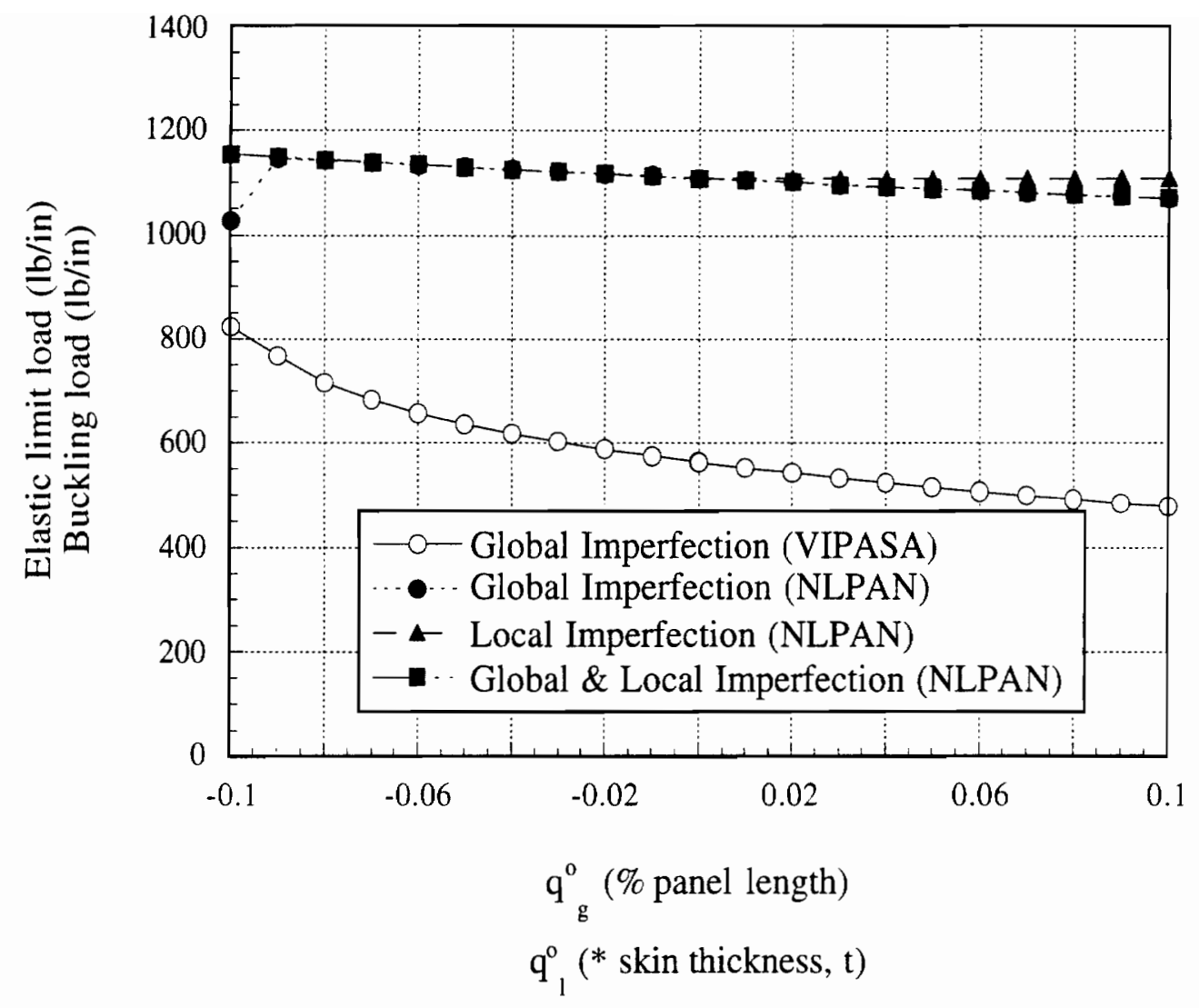

FIGURE 47. Imperfection sensitivity of the NLPANOPT flange design with initial global imperfection $($ Design load $=1000 \mathrm{lb} / \mathrm{in})$. 
imperfections. Investigation of imperfection sensitivity of the continuous design (shown in Figure 48), the panel still carries the required load for all global imperfection amplitudes.

TABLE 14. Summary of NLPANOPT flange designs with an initial global imperfection for $\mathrm{N}_{\mathrm{x}}=1000 \mathrm{lb} / \mathrm{in}$.

\begin{tabular}{|c|c|c|c|c|}
\hline & Design 1 & Design 2 & Design 3 & Design 4 \\
\hline \hline $\mathrm{b}$ (in.) & 1.60160 & 1.64495 & 1.84927 & 1.77728 \\
\hline $\mathrm{t}_{1}\left(0^{\circ}{ }_{\text {skin }}\right)$ & 1 & 1 & 1 & 1 \\
\hline $\mathrm{t}_{2}\left(90^{\circ}{ }_{\text {skin }}\right)$ & 1 & 1 & 1 & 1 \\
\hline $\mathrm{t}_{3}\left( \pm 45^{\circ}{ }_{\text {skin }}\right)$ & 1 & 1 & 1 & 1 \\
\hline $\mathrm{t}_{4}\left(0^{\circ}\right.$ flange $)$ & 1 & 1 & 1 & 1 \\
\hline $\mathrm{t}_{5}\left(90^{\circ}\right.$ flange $)$ & 1 & 1 & 1 & 1 \\
\hline $\mathrm{t}_{6}\left( \pm 45^{\circ}\right.$ flange $)$ & 1 & 1 & 1 & 1 \\
\hline $\mathrm{t}_{7}\left(0^{\circ}{ }_{\text {blade }}\right)$ & 7 & 6 & 4 & 7 \\
\hline $\mathrm{t}_{8}\left(90^{\circ}{ }_{\text {blade }}\right)$ & 1 & 1 & 1 & 1 \\
\hline $\mathrm{t}_{9}\left( \pm 45^{\circ}{ }_{\text {blade }}\right)$ & 1 & 2 & 2 & 1 \\
\hline $\begin{array}{c}\text { Unit Weight } \\
(\text { lbs. })\end{array}$ & 1.61701 & 1.68540 & 1.63766 & 1.67481 \\
\hline$\lambda_{\text {global }}$ & 1.18 & 1.20 & 1.28 & 1.53 \\
\hline$\lambda_{\text {local }}$ & 0.564 & 0.562 & 0.497 & 0.594 \\
\hline $\mathrm{m}_{\text {local }}$ & 13 & 14 & 13 & 13 \\
\hline
\end{tabular}

The rounded-off NLPANOPT design with an initial global imperfection listed in Table 13 is the minimum-weight design from a number of designs obtained from various initial design configurations. Four local optima were identified and the rounded-off designs are listed in Table 14 with the design listed in Table 13 shown as Design 1. All four designs buckle locally between $500 \mathrm{lb} / \mathrm{in}$ and $600 \mathrm{lb} / \mathrm{in}$. The panel response for each design is shown in Figure 49. The global end load is plotted as a function of end shortening. Design 


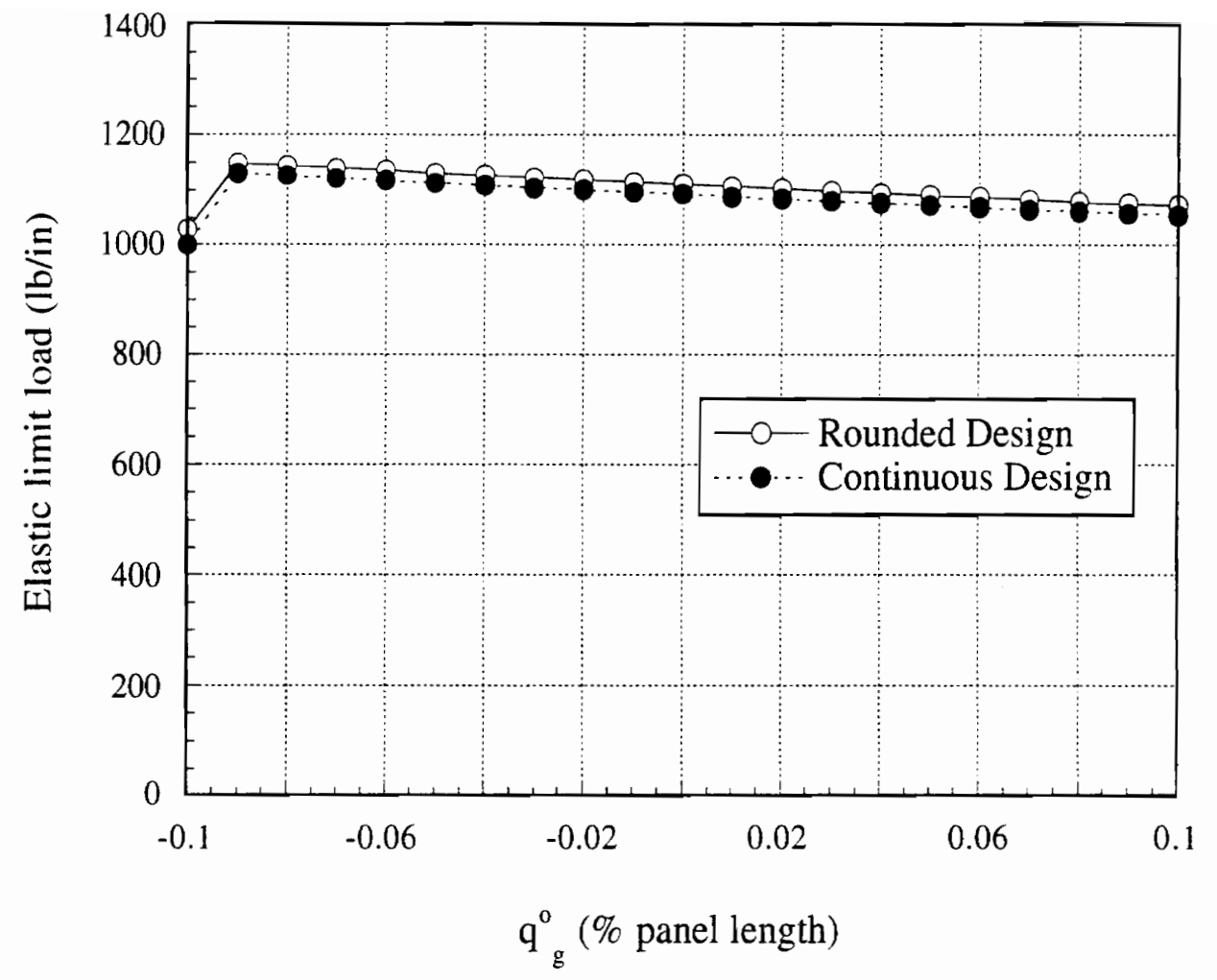

FIGURE 48. Imperfection sensitivity of the continuous NLPANOPT flange design with initial imperfection to global imperfection shapes (Design load $=$ $1000 \mathrm{lb} / \mathrm{in})$. 


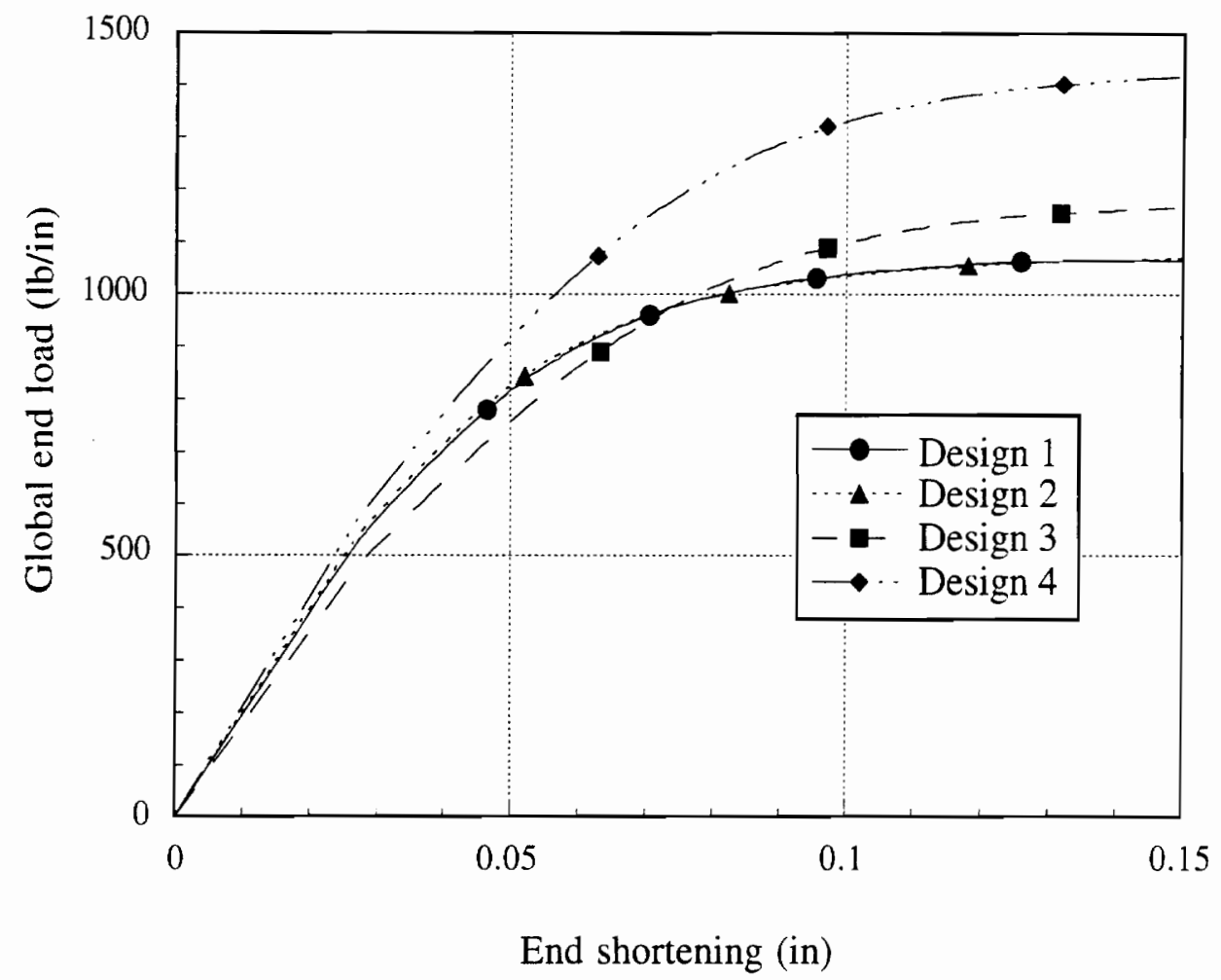

Figure 49. Load vs. End Shortening for NLPANOPT flange designs with initial global imperfection $\left(N_{x}=1000 \mathrm{lb} / \mathrm{in}\right)$. 
1 and Design 2 behave identically, while Design 3 is initially less stiff and Design 4 is initially more stiff. Even though there is a difference in axial stiffness, all the designs use the local postbuckling performance of the stiffened panel.

Comparing the rounded-off NLPANOPT designs for the blade-stiffened configuration (Section 4.1.2) with the rounded-off NLPANOPT designs for the T-stiffened configuration, an increase in weight is naturally observed with the addition of the flange. For the designs with no initial imperfections, the T-stiffened panel weighs $14 \%$ more than the blade-stiffened panel. The weight-savings for each of these designs compared to their respective PASCO designs, however, is the same (28\%). The blade height for the T-stiffened panel is reduced $3.6 \%$ compared to the blade-stiffened panel and requires one less $0^{\circ}$ ply thickness in the blade. Also, with the addition of the flange, the minimum-weight design buckles at $48 \%$ of the design load rather than $29 \%$ of the design load with no flange.

For the designs with an initial global imperfection shape, the T-stiffened panel weighs $19 \%$ more than the blade-stiffened panel. The weight-savings for the blade-stiffened panel compared to its PASCO design (44\%) is greater than that for the T-stiffened panel compared to its PASCO design (21\%). The blade height for the T-stiffened panel is reduced $10.6 \%$ compared to the blade-stiffened panel but requires one more $0^{\circ}$ ply thickness in the blade. Also, with the addition of the flange, the minimum-weight design buckles at $56 \%$ of the design load rather than $29 \%$ of the design load with no flange. With the addition of the flange, possible deformation constraints can be lessened since the designs buckle at higher loads.

For the NLPANOPT flange designs, the flange width was not included as a design variable. The effect of decreasing the flange width was investigated by redesigning the 
NLPANOPT designs for a flange width of 0.5 in. The resulting designs were almost identical to the designs presented with a flange width of $1.0 \mathrm{in}$. The thickness of the flange remained to be minimum and plies were added only to the $0^{\circ}$ ply in the blade. 


\subsection{Concluding Remarks}

\subsection{Conclusions}

A preliminary design procedure has been developed for the minimum-weight design of compressively loaded stiffened panels. The design procedure is a combination of an integrated analysis and the optimization code ADS. The integrated analysis consists of two codes, NLPAN and PASCO. NLPAN is a geometrically nonlinear analysis that uses a perturbation approach based on the use of a series of buckling mode shapes to represent nonlinear displacements. The buckling mode shape information is obtained using the VIPASA analysis within the PASCO design code. An automated selection procedure for the set of buckling mode shapes has been established based on the expectation of modal interaction type behavior.

A blade-stiffened panel was designed for four load levels: $\mathrm{N}_{\mathrm{x}}=100 \mathrm{lb} / \mathrm{in}, 1000 \mathrm{lb} / \mathrm{in}, 5000$ $\mathrm{lb} / \mathrm{in}$, and $10000 \mathrm{lb} / \mathrm{in}$. Comparisons between the nonlinear design procedure and the linear design procedure PASCO were made. No significant advantages from using the nonlinear analysis were identified for the lightest design load, $\mathrm{N}_{\mathrm{x}}=100 \mathrm{lb} / \mathrm{in}$.

For the next design load, $\mathrm{N}_{\mathrm{x}}=1000 \mathrm{lb} / \mathrm{in}$, the rounded-off NLPANOPT design with no initial imperfections was $28 \%$ lighter than the corresponding PASCO design. The rounded- 
off NLPANOPT design with an initial global imperfection was $38 \%$ lighter than the corresponding PASCO design. Also, the rounded-off NLPANOPT designs were less imperfection sensitive than the rounded-off PASCO designs in the sense that these panels carried the design load for the entire range of imperfection shapes and amplitudes investigated. The continuous designs from PASCO and the continuous design with no account for initial imperfections from NLPANOPT did not carry the design load for the range of imperfections investigated. The continuous NLPANOPT design that included an initial global imperfection, however, carried the design load for the entire imperfection range.

Weight-savings and imperfection insensitivity was also seen for the NLPANOPT designs with a $\mathrm{N}_{\mathrm{x}}=5000 \mathrm{lb} / \mathrm{in}$ design load. Both rounded-off NLPANOPT designs were around $32 \%$ lighter than the corresponding PASCO design. Additionally, a number of local minimum-weight designs were presented for the NLPANOPT designs. For the panel designed with no account for imperfections, the three designs identified ranged from possessing highly local postbuckling behavior to global postbuckling behavior. This type of range in behavior allows alternative choices in designs that are based not solely on weight-savings but also on possible deformation constraints. The lowest weight design, however, was the one which possessed the highest amount of local postbuckling behavior. For the panel designed with an initial global imperfection, two local postbuckled designs were identified. The first design, was more locally postbuckled (buckling at $14.5 \%$ of the design load) than the second design (buckling at $90 \%$ of the design load). The first design was $34 \%$ lighter than the second design.

For the highest design load, $\mathrm{N}_{\mathrm{x}}=10000 \mathrm{lb} / \mathrm{in}$, no advantage was seen in designing the panel with NLPANOPT and no initial imperfections. However, when an initial global imperfection was included, the NLPANOPT design was $16 \%$ lighter than the corresponding PASCO design panel. The imperfection sensitivity of this NLPANOPT design was 
also eliminated.

A shortcoming of the NLPANOPT design procedure was identified with regards to the need for prescribing the laminate stacking sequences. An investigation into the effect of changing the skin laminate stacking sequence for the $N_{x}=1000 \mathrm{lb} / \mathrm{in}$ design load showed that lower weight designs are possible by changing the stacking sequence.

A T-stiffened panel was designed for $a \mathrm{~N}_{\mathrm{x}}=1000 \mathrm{lb} / \mathrm{in}$ design load. The NLPANOPT designs were lower in weight than the PASCO designs and buckled locally before the design load. The NLPANOPT design with an initial global imperfection was the least imperfection sensitive whether considering its rounded-off design or continuous design. The blade-stiffened NLPANOPT panels, naturally lighter, were found to be in a higher state of local postbuckling compared to the T-stiffened panels.

\subsection{Future Work}

With regards to changes to the design procedure, a genetic algorithm (GA) will be incorporated as the optimization code into NLPANOPT. The GA will eliminate the need for rounding-off designs and permit a constraint to be placed on the number of similar ply orientations allowed in a row. Finally, the genetic algorithm will determine the global optimum stacking sequence, automatically eliminating unnecessary plies.

An improvement to the method in which the nonlinear analysis accounts for imperfections is as follows. Currently, when imperfections are included in an analysis, no imperfection is applied to the PASCO generated buckling mode shapes. A more accurate representation of the nonlinear deformations may be possible if similar bowing imperfections are applied to the panel within PASCO when the buckling mode shapes are determined.

Further investigation into design trends would be useful. For example, a study into the 
effect of stiffener spacing on panel weight, the effect of transverse loading on panel weight, or the effects on the panel weight of accounting for a range of initial global imperfection amplitudes. Also, with the completion of the simple geometries presented, more complex geometries should be studied, such as I- or J-stiffened panels. For these cases, the accuracy of the current mode set selection strategy would need to be investigated. 


\subsection{References}

1. Thompson, J. M. T. and Lewis, G. M., "On the Optimum Design of Thin-walled Compression Members,” J. Mech. Phys. Solids, Vol. 20, 1972, pp. 101-109.

2. Almroth, B. O., Brogan, F. A., and Stanley, G. M., "User's Manual for STAGS - Volume I: Theory," NASA CR-165670, March, 1978.

3. Almroth, B. O., Brogan, F. A., and Stanley, G. M., "Structural Analysis of General Shells - Volume II: User Instructions for STAGSC-1," LMSC-D633873, Applied Mechanics Lab., Lockheed Palo Alto Research Lab., Palo Alto, CA, December, 1982, updated 1985.

4. Stoll, F., Gürdal, Z., and Starnes, J. H. Jr., "A Method for the Geometrically Nonlinear Analysis of Compressively Loaded Prismatic Composite Structures," CCMS-91-03 (VPI-E-91-01), Center for Composite Materials and Structures, Virginia Polytechnic Institute and State University, Blacksburg, VA, 1991.

5. Wittrick, W. H., and Williams, F. W., "Buckling and Vibration of Anisotropic or Isotropic Plate Assemblies under Combined Loadings," Int. J. Mechanical Science, Vol. 16, 1974, pp. 209-239.

6. Williams, F. W. and Anderson, M. E., "User's Guide to VIPASA (Vibration and Instability of Plate Assemblies Including Shear and Antisotropy)," Department of Civil Engineering, University of Birmingham, 1973.

7. Stroud, W. J., and Anderson, M. S., "PASCO: Structural Panel Analysis and Sizing Code, Capability and Analytical Foundations," NASA TM-80181, November, 1981.

8. Anderson, M. S. and Stroud, W. J., "PASCO: Structural Panel Analysis and Sizing Code, User's Manual," NASA TM-80182, 1981.

9. Vanderplaats, G. N., "ADS-A FORTRAN Program for Automated Design SynthesisVersion 1.10," NASA-CR-177985, 1985.

10. Vanderplaats, G. N. and Moses, F., "Structural Optimization by Methods of Feasible Directions," Computers and Structures, Vol. 3, July 1973, pp. 739-755.

11. EISI/SPAR Reference Manual, System Level 103, Engineering Information Systems Inc., San Jose, CA, January 1979. 
12. Stroud, W. J., Greene, W. H., and Anderson, M. S., "Buckling Loads for Stiffened Panels Subjected To Combined Longitudinal Compression and Shear Loadings: Results Obtained with PASCO, EAL, and STAGS Computer Programs," NASA TM-83194, October 1981.

13. Williams, F. W., Kennedy, D., and Anderson, M. S., " Analysis Features of VICONOPT, an Exact Buckling and Vibration Program for Prismatic Assemblies of Anisotropic Plates," AIAA/ASME/ASCE/AHS/ASC 31st Structures, Structural Dynamics and Materials Conference, Long Beach, CA, April 2-4, 1990, pp. 920-929.

14. Butler, R. and Williams, F. W., " Optimum Design Features of VICONOPT, an Exact Buckling Program for Prismatic Assemblies of Anisotropic Plates," AIAA/ASME/ ASCE/AHS/ASC 31st Structures, Structural Dynamics and Materials Conference, Long Beach, CA, April 2-4, 1990, pp. 1289-1299.

15. Swanson, G. D., Gürdal, Z., and Starnes, J. H. Jr., "Structural Efficiency Study of Graphite-Epoxy Aircraft Rib Structures," AIAA Paper No. 88-2218, AIAA/ASME/ ASCE/AHS/ASC 29th Structures, Structural Dynamics and Materials Conference, Williamsburg, VA, April 18-20, 1988.

16. Thompson, J. M. T. and Hunt, G. W., "Dangers of Structural Optimization," Engineering Optimization, Vol. 1, 1974, pp. 99-110.

17. Koiter, W. T. and Skaloud, M., Interventions, "Comportment Postcritique des Plaques Utilisees en Construction Metalique," Memoires de la Societe Royale des Sciences de Liege, $5^{\mathrm{me}}$ serte, tome VIII, fasc. 5, pp. 64-68, 103, 104.

18. Graves-Smith, T. R., "The Ultimate Strength of Locally Buckled Columns of Arbitrary Length"” Thin Walled Steel Construction (Ed. by K. C. Rockey and H. V. Hill), Crosby Lockwood, London, 1967.

19. van der Neut, A., "The Interaction of Local Buckling and Column Failure of Thinwalled Compression Members," Proc. 12th Int. Cong. Appl. Mech., Stanford University, 1969, pp. 389-399.

20. van der Neut, A., "The Sensitivity of Thin-walled Compression Members to Column Axis Imperfection," Int. J. Solids Structures, Vol. 9, 1973, pp. 999-1011.

21. Tvergaard, V., "Imperfection-Sensitivity of a Wide Integrally Stiffened Panel Under Compression," Int. J. Solids Structures 9, 1973, pp. 177-192.

22. Koiter, W. T. and Pignataro, M., "An Alternative Approach to the Interaction Between Local and Overall Buckling of Stiffened Panels," IUTAM Symposium, Cambridge, Buckling of Structures, (Ed. by B. Budiansky), Springer-Verlag, Berlin, 1976, pp. 133148.

23. van der Neut, A., "Mode Interaction with Stiffened Panels," IUTAM Symposium, Cambridge, Buckling of Structures, (Ed. by B. Budiansky), Springer-Verlag, Berlin, 1976, pp. 117-132. 
24. Thompson, J. M. T., Tulk, J. D., and Walker, A. C., "An Experimental Study of Imperfection-Sensitivity in the Interactive Buckling of Stiffened Plates," IUTAM Symposium, Cambridge, Buckling of Structures, (Ed. by B. Budiansky), Springer-Verlag, Berlin, 1976, pp. 149-159.

25. Tulk, J. D., and Walker, A. C., "Model Studies of the Elastic Buckling of a Stiffened Palte," J. of Strain Analysis, Vol. 11, No. 3, 1976, pp. 137-143.

26. Agarwal, B. L., "Postbuckling Behavior of Composite Shear Webs," AIAA Journal, July 1981, pp. 933-939.

27. Sridharan, S., "Doubly Symmetric Interactive Buckling of Plate Structures," Int. J. of Solids and Structures, Vol. 19, No. 7, 1983, pp. 625-641.

28. Sridharan, S. and Ali, M. A., "Interactive Buckling in Thin-Walled Beam-Columns," $J$. Engng. Mech. ASCE 111 (12), 1985, pp. 1470-1486.

29. Ali, M. A. and Sridharan, S., "A Versatile Model for Interactive Buckling of Columns and Beam-Columns," Int. J. Solids Structures, Vol. 24, No. 5, 1988, pp. 481-496.

30. Ali, M. A. and Sridharan, S., "A Special Beam Element for the Analysis of ThinWalled Structural Components," Int. J. Numerical Methods Engng., Vol. 28, 1989, pp. 1733-1747.

31. Peng, M. and Sridharan, S., "Optimized Design of Stiffened Panels Subject to Interactive Buckling," AIAA/ASME/ASCE/AHS/ASC 31st Structures, Structural Dynamics and Materials Conference, Long Beach, CA, April 2-4, 1990, pp. 1279-1288.

32. Peng, M. and Sridharan, S., "An Optimization Strategy For Wide Stiffened Plates Subject to Interaction of Local and Overall Buckling," Computer \& Structures, Vol. 41, No. 2, 1991, pp.233-243.

33. Powell, M. J. D., "Algorithms for Nonlinear Constraints that Use Lagrangian Functions," Math. Programming 14, 1978, pp. 224-228.

34. Dickson, J. N. and Wang, J. T. S., "Design of Stiffened Composite Panels in the PostBuckling Range," Proc. 4th Conference on Fibrous Composites in Structural Design, San Diego, CA, Nov. 14-17, 1978, pp. 313-327.

35. Dickson, J. N. and Biggers, S. B., "A Preliminary Design Procedure for Composite Panels with Open-Section Stiffeners Loaded in the Post-Buckling Range," In Advances in Composite Materials (Ed. by A. R. Bunsell, et al.), Pergamon Press, Oxford, 1980, pp. 812-825.

36. Biggers, S. B. and Dickson, J. N., "POSTOP: Postbuckled Open-Stiffener Optimum Panels-User's Manual,” NASA CR-172260, N84-18682, January, 1984.

37. Timoshenko, S. P. and Gere, J. M., Theory of Elastic Stability, Second Edition, McGraw-Hill Book Company Inc., 1961.

38. Bushnell, D., "PANDA2-Program for Minimum Weight Design of Stiffened, Composite, Locally Buckled Panels," Computers \& Structures, Vol. 25, No. 4, 1987, pp. 469605. 
39. Bushnell, D., "Optimization of , Composite, Stiffened, Imperfect, Panels Under Combined Loads for Service in the Postbuckling Regime," Comput. Meth. Appl. Mech. Engng. 103, 1993, pp. 43-114.

40. Stoll, F., "An Implementation of Solution Strategies for the Analysis of Complex Nonlinear Equilibrium Behavior," Int. J. of Non-Linear Mechanics, Vol. 29, No. 2, March, 1994, pp. 109-122.

41. Chia, C., Nonlinear Analysis of Plates, McGraw Hill, Inc., 1980.

42. Jones, R. M., Mechanics of Composite Materials, Hemisphere Publishing Corp., New York, 1975.

43. Riks, E., "An Incremental Approach to the Solution of Snapping and Buckling Problems," Int. J. Solids and Structures, Vol. 15, 1979, pp. 529-551.

44. Thurston, G. A., Brogan, F. A., and Stehlin, P., "Postbuckling Analysis Using a General-Purpose Code," AIAA Journal, Vol. 24, No. 6, June 1986, pp. 1013-1020.

45. Lucas, S.H. and Davis, R.C., "MacPASCO: A Macintosh-Based, Interactive Graphic Preprocessor for Structural Analysis and Sizing," AIAA Paper No. 91-1208, AIAA/ ASME/ASCE/AHS/ASC 32nd Structures, Structural Dynamics and Materials Conference, Baltimore, MD, April 8-10, 1991.

46. Nagendra, S., Haftka, R. T., and Gurdal, Z., "Stacking Sequence Optimization of Simply Supported Laminates with Stability and Strain Constraints," AIAA Journal, Vol. 30, No. 8, 1992, pp. 2132-2137. 


\section{Vitae}

The author was born on September 10, 1968 in Pittsburgh, Pennsylvania to Robert J. and Amiela J. Perry. Upon completing high school in 1986 she began studies in the Department of Engineering at The Pennsylvania State University in University Park, Pennsylvania. In her junior year, she entered the Department of Aerospace Engineering from which she received a B.S. in May 1990. That fall she enrolled in the NASA-Virginia Tech Composites Program to pursue her masters degree. 